\title{
AN EXPERIMENTAL INVESTIGATION OF CHURNING POWER LOSSES OF A GEARBOX
}

\section{THESIS}

Presented in Partial Fulfillment of the Requirements for The Degree of Master of Science in the Graduate School of the Ohio State University

\author{
By \\ Joseph H. Polly, B.S. \\ Graduate Program in Mechanical Engineering \\ $* * * * *$ \\ The Ohio State University \\ 2013 \\ Thesis Committee: \\ Dr. Ahmet Kahraman, Advisor \\ Dr. Donald Houser
}


(C) Copyright by:

Joseph H. Polly

2013 


\begin{abstract}
In this study, load-independent (spin) power losses of a gearbox operating under dip-lubrication conditions are investigated experimentally. A family of final drive helical gear pairs from an automotive transmission is considered as the example for this investigation. A dedicated gearbox is designed and fabricated to operate a single gear or a gear pair under given speed conditions. The test gearbox is incorporated with a highspeed test bed with power loss measurement capability. A test matrix that consists of sets of tests with (i) single spur, helical gears, or disks with no teeth, and (ii) helical gear pairs of varying gear ratios is executed with three different transmission fluids at various temperatures and immersion depths. Power losses from single gear and gear pair tests at identical operating conditions are compared to break down the total spin loss to its main components, namely gear drag loss, gear mesh pocketing loss, and bearing/seal loss. In addition, the space around the gears within the gearbox will be altered to quantify any influences of enclosures and peripheral shrouds on the spin losses of a rotating gear.
\end{abstract}


Dedicated to my family: past, present, and future 


\section{ACKNOWLEDGEMENTS}

I would like to offer the highest level of gratitude to my advisor, Dr. Ahmet Kahraman, for the wonderful opportunity to learn and work in the GearLab. His patient guidance through this project has taught me a great deal and will provide a solid foundation for a successful career. I would like to thank Sam Shon, David Talbot, and my other GearLab colleagues for making this a truly enjoyable experience. My thanks go to my project sponsor, GM Powertrain, in particular Avinash Singh and Hai Xu for their encouragement and financial support. I would also like to thank Dr. Donald Houser for his input and participation in this thesis and defense. Additionally, I thank Satya Seetharaman, Jonny Harianto, Chad Bivens, Richard Treynor, and Will Estep for all your support and assistance throughout this project.

Special thanks go to my parents, Mark and Beth, for whom I am eternally grateful for their guidance and encouragement throughout my life. They have provided me with the capabilities and confidence to be successful in all my endeavors. I would like to thank my sisters, Emma and Lauren, for their support. I would also like to thank my grandparents, Dave and Judy, for their willingness to listen and the valuable life lessons they have shared with me over the years. 


\section{VITA}

July 29, 1988

Born - Cleveland, Ohio

June 2010 - December 2010

Engineering Intern

NASA Glenn Research Center

Cleveland, Ohio

Sept. 2009 - May 2011

Undergraduate Research Assistant

The Ohio State University

Dept. of Mech. Engineering

Columbus, Ohio

June 2011

B.S. Mechanical Engineering

The Ohio State University

Columbus, Ohio

June 2011 - January 2013 ........................................... Graduate Research Associate

The Ohio State University

Dept. of Mech. Engineering

Columbus, Ohio

\section{PUBLICATIONS}

1. Handschuh, R. F., Polly, J., Morales, W., "Gear Mesh Loss-of-Lubrication Experiments and Analytical Simulation." 67th Annual Forum and Technology Display Sponsored by the American Helicopter Society, Virginia Beach, Virginia, 2011

\section{FIELD OF STUDY}

Major Field: Mechanical Engineering 


\section{TABLE OF CONTENTS}

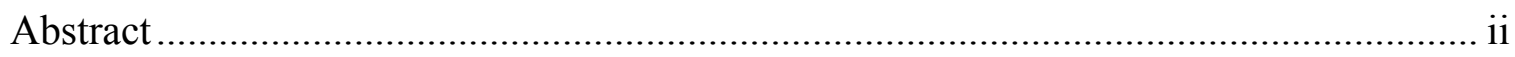

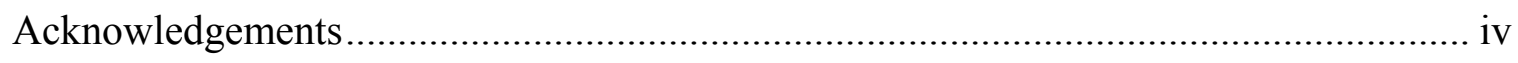

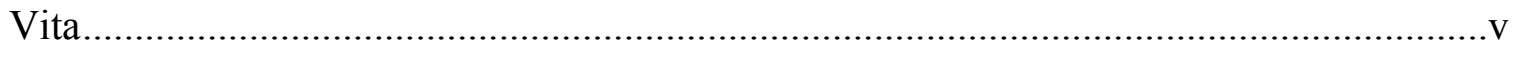

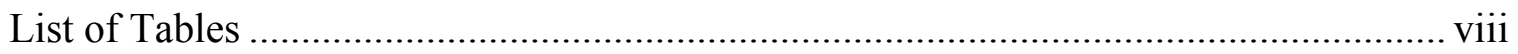

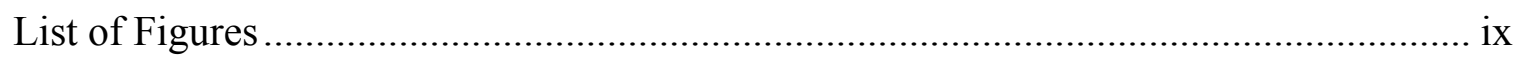

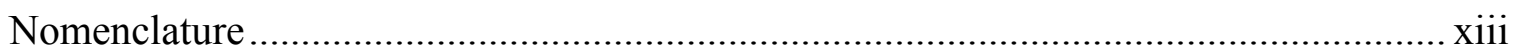

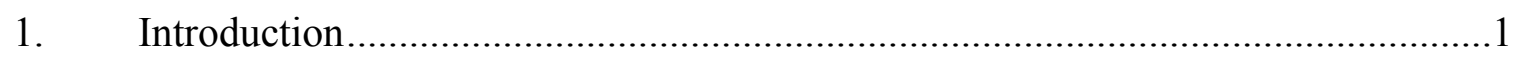

1.1 Background and Motivation ......................................................................

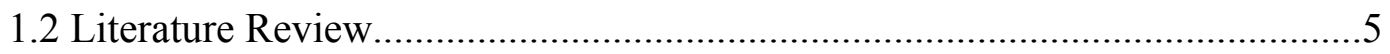

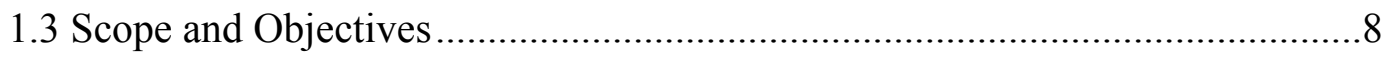

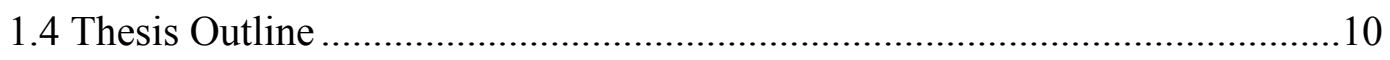

2. Experimental Test Methodology ...................................................................

2.1 Design and Development of the Test Gearbox ................................................11

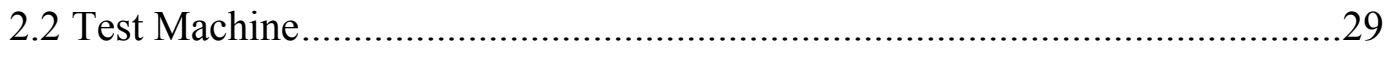

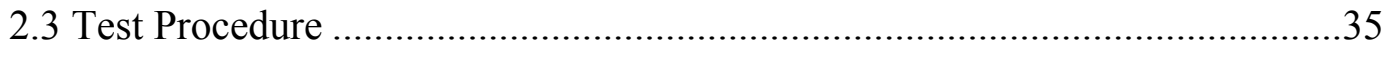

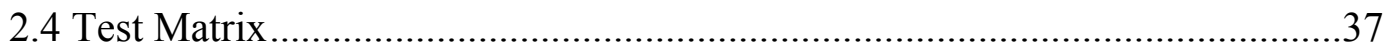




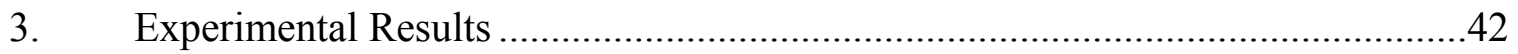

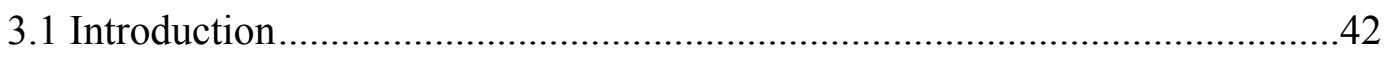

3.1.1 Repeatability of Measurements..........................................................4

3.1.2 Bearing and Seal Power Loss .............................................................4

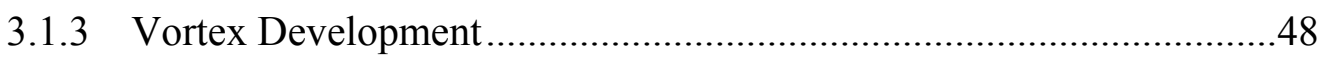

3.2 Drag Losses of a Single Gear......................................................................

3.2.1 Effect of Ring Gear Size .................................................................51

3.2.2 Effect of Lubricant Type and Static Oil Level....................................51

3.2.3 Effect of Lubricant Temperature ………………………………......58

3.2.4 Influence of Gear Teeth and Helix Angle............................................61

3.3 Gear Pair Experiments ...............................................................................67

3.3.1 Effect of Gear Ratio .......................................................................67

3.3.2 Influence of Circumferential Pinion Position .......................................70

3.3.3 Components of Spin Power Loss .......................................................74

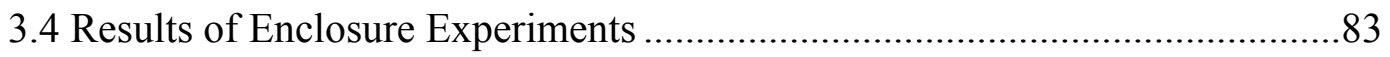

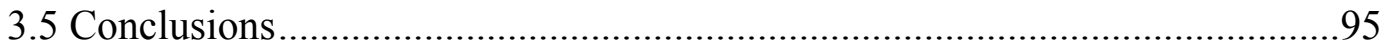

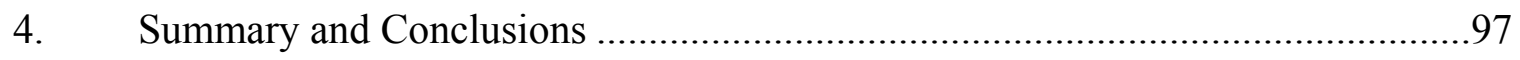

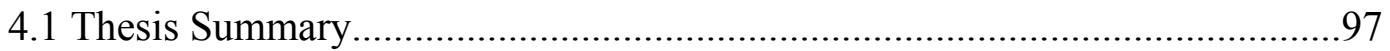

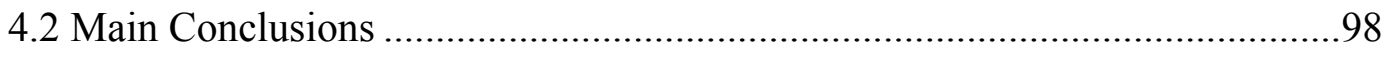

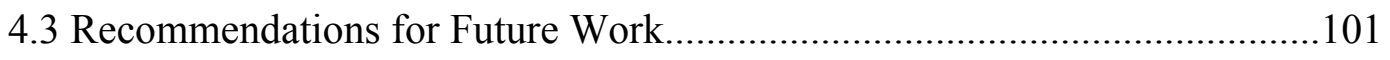

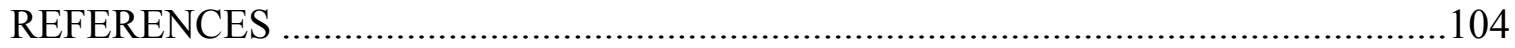




\section{LIST OF TABLES}

\section{Table:}

2.1 Basic design parameters of final drive gear pairs used in this study ....................13

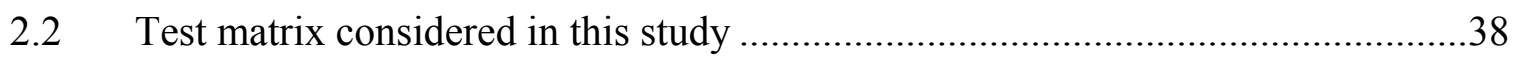




\section{LIST OF FIGURES}

1.1 Main components of power losses of a geared system [1] ..................................

2.1 Pictures of (a) gear pair \#1, (b) gear pair \#2, (c) gear pair \#3 .............................14

2.2 (a) Pinions and (b) ring gears of the test gear pairs .......................................... 16

2.3 Solid model of the test gearbox used in this study.

Side and top covers are made of polycarbonate

material for viewing purposes.

2.4 Cross-sectional drawings of the test gearbox (a)

with both gears assembled, and (b) with only the ring gear assembled................18

2.5 A picture of the assembled test gearbox ......................................................... 19

2.6 (a) Helical ring gear, (b) spur ring gear (c) ring gear blank with no teeth, and $(\mathrm{d}, \mathrm{e})$ side views comparing each variation.

2.7 (a) Inboard deep-groove ball bearings, and

(b) outboard deep-groove ball bearings used to support

both shafts of the gearbox. All dimensions are in $\mathrm{mm}$

2.8 The views of the test gearbox in circumferential

pinion positions of (a) $\phi=0^{\circ}$; (b) $\phi=90^{\circ}$; (c) $\phi=180^{\circ}$

2.9 Circumferential pinion position angles $\phi$ achievable by the test gearbox

2.10 (a) Parameters defining of the static oil level parameter $\bar{h}$ and

(b) static oil levels considered in this study .28

2.11 (a) Side and (b) frontal view schematics of the gearbox defining the enclosure configurations used in this study .30 
2.12 (a) Front and (b) top view of a set of enclosures assembled in the test gearbox.

2.13 A picture of the high speed efficiency test machine ...............................................33

2.14 Top-view schematic of the test set-up with its key components labeled.................34

2.15 (a) Kinematic viscosity and (b) density as a function of temperature for the test lubricants considered in the study

3.1 Sample segments of measured $\Omega_{r}, T$ and oil temperature time histories from a test at set values of $\Omega_{r}=1500$ and $90^{\circ} \mathrm{C}$ with Lubricant B

3.2 Repeatability of $\bar{P}_{S}$ measurements with ring gear $\# 3$

Operated in lubricant $\mathrm{B}$ at $60^{\circ} \mathrm{C}$ and $\bar{h}=1.00$

3.3 Measured input shaft bearing and seal power loss from wet and dry bearing loss tests with lubricant $\mathrm{B}$ at $60^{\circ} \mathrm{C}$.

Also shown is the comparison formulae of Harris and Kotzalas [35].

3.4 Examples of oil flow (a) without a vortex and (b) with a vortex.

3.5 Influence of ring gear diameter on $\bar{P}_{S}$ at (a) $\bar{h}=0.05$, (b) $\bar{h}=1.0$, (c) $\bar{h}=1.5$, and (d) $\bar{h}=2.0$. Lubricant B at $60^{\circ} \mathrm{C}$

3.6 Combined influences of lubricant type and $\bar{h}$ on $\bar{P}_{S}$ at $60^{\circ} \mathrm{C}$ and (a) $\Omega_{r}=250 \mathrm{rpm}$, (b) $\Omega_{r}=500 \mathrm{rpm}$, (c) $\Omega_{r}=750 \mathrm{rpm}$, (d) $\Omega_{r}=1000 \mathrm{rpm}$, (e) $\Omega_{r}=1500 \mathrm{rpm}$ and (f) $\Omega_{r}=2000 \mathrm{rpm}$.

3.7 Effect of bulk lubricant temperature on $\bar{P}_{S}$ with Lubricant B at (a) $\bar{h}=0.05$, (b) $\bar{h}=1.0$, (c) $\bar{h}=1.5$, and (d) $\bar{h}=2.0$

3.8 Effect of gear teeth on $\bar{P}_{S}$ within a range of $\Omega_{r}$ at

(a) $\bar{h}=0.05$, (b) $\bar{h}=0.5$, (c) $\bar{h}=1.0$, and (d) $\bar{h}=2.0$.

Lubricant $\mathrm{B}$ at $60^{\circ} \mathrm{C}$ 
3.9 Effect of gear teeth on $\bar{P}_{S}$ within a range of $\bar{h}$ at (a) $\Omega_{r}=500 \mathrm{rpm}$, (b) $\Omega_{r}=1000 \mathrm{rpm}$, (c) $\Omega_{r}=1500 \mathrm{rpm}$, and (d) $\Omega_{r}=2000 \mathrm{rpm}$

3.10 Influence of gear ratio $n$ on $\bar{P}_{S}$ within a range of $\bar{h}$ at (a) $\Omega_{r}=500 \mathrm{rpm}$, (b) $\Omega_{r}=1000 \mathrm{rpm}$, (c) $\Omega_{r}=1500 \mathrm{rpm}$, and (d) $\Omega_{r}=2000 \mathrm{rpm}$. Lubricant B at $90^{\circ} \mathrm{C}, \phi=90^{\circ}$

3.11 Effect of $\phi$ on $\bar{P}_{S}$ within a range of $\bar{h}$ at (a) $\Omega_{r}=500 \mathrm{rpm}$,

(b) $\Omega_{r}=1000 \mathrm{rpm}$, (c) $\Omega_{r}=1500 \mathrm{rpm}$, and (d) $\Omega_{r}=2000 \mathrm{rpm}$.

Lubricant B at $90^{\circ} \mathrm{C}$

3.12 Components of $\bar{P}_{S}$ with gear pair \#3 and the pinion at

$\phi=0^{\circ}$ for (a) $\bar{h}=0.5$, (b) $\bar{h}=1.0$, (c) $\bar{h}=1.5$, and

(d) $\bar{h}=2.0$. Lubricant B at $60^{\circ} \mathrm{C}$.

3.13 Components of $\bar{P}_{S}$ with gear pair \#3 and the pinion at $\phi=90^{\circ}$ for (a) $\bar{h}=0.5$, (b) $\bar{h}=1.0$, (c) $\bar{h}=1.5$, and (d) $\bar{h}=2.0$. Lubricant $\mathrm{B}$ at $60^{\circ} \mathrm{C}$ .78

3.14 Components of $\bar{P}_{S}$ with gear pair \#3 with the pinion at $\phi=180^{\circ}$ for (a) $\bar{h}=0.5$, (b) $\bar{h}=1.0$, (c) $\bar{h}=1.5$, and (d) $\bar{h}=2.0$. Lubricant $\mathrm{B}$ at $60^{\circ} \mathrm{C}$

3.15 An example lubricant flow behavior in an enclosed environment ( $\bar{h}=1.0$ and $\left.\Omega_{r}=1000 \mathrm{rpm}\right)$

3.16 Effect of $p$ on $\bar{P}_{S}$ from a ring gear test with $s=12 \mathrm{~mm}$ at (a) $\bar{h}=0.5$, (b) $\bar{h}=1.0$, (c) $\bar{h}=1.5$, and (d) $\bar{h}=2.0$. Ring gear \# 3 with lubricant $\mathrm{B}$ at $60^{\circ} \mathrm{C}$ .86

3.17 Effect of $p$ on $\bar{P}_{S}$ from a ring gear test with $s=1 \mathrm{~mm}$ at (a) $\bar{h}=0.5$, (b) $\bar{h}=1.0$, (c) $\bar{h}=1.5$, and (d) $\bar{h}=2.0$. Ring gear \# 3 with lubricant $\mathrm{B}$ at $60^{\circ} \mathrm{C}$ .88 
3.18 Effect of $s$ on $\bar{P}_{S}$ from a ring gear test with $p=12 \mathrm{~mm}$ at (a) $\Omega_{r}=500 \mathrm{rpm}$, (b) $\Omega_{r}=1000 \mathrm{rpm}$, (c) $\Omega_{r}=1500 \mathrm{rpm}$, and (d) $\Omega_{r}=2000 \mathrm{rpm}$. Ring gear \# 3 with lubricant B at $60^{\circ} \mathrm{C}$

3.19 Effect of $s$ on $\bar{P}_{S}$ from a ring gear test with $p=5 \mathrm{~mm}$ at (a) $\Omega_{r}=500 \mathrm{rpm}$, (b) $\Omega_{r}=1000 \mathrm{rpm}$, (c) $\Omega_{r}=1500 \mathrm{rpm}$, and (d) $\Omega_{r}=2000 \mathrm{rpm}$. Ring gear \# 3 with lubricant B at $60^{\circ} \mathrm{C}$

3.20 Combined effects of $p$ and $s$ on $\bar{P}_{s} \mathrm{~mm}$ at (a) $\Omega_{r}=1000 \mathrm{rpm}$ and (b) $\Omega_{r}=2000 \mathrm{rpm}$. Ring gear \# 3 with lubricant $\mathrm{B}$ at $60^{\circ} \mathrm{C}$ .94 


\section{NOMENCLATURE}

$\begin{array}{ll}\text { Symbol } & \text { Definition } \\ h & \text { Height of static lubricant level from bottom of gear } \\ \bar{h} & \text { Normalized static lubricant level, } h / r \\ n & \text { Gear ratio } \\ p & \text { Peripheral enclosure gap } \\ P_{b} & \text { Bearing power loss } \\ P_{p d} & \text { Spin power loss due to pinion } \\ P_{p o c} & \text { Spin power loss due to pocketing } \\ P_{r d} & \text { Spin power loss due to ring gear drag } \\ P_{r e f} & \text { Reference spin power loss } \\ P_{s} & \text { Total spin power loss } \\ P_{S} & \text { Normalized spin power loss, } P_{S} / P_{r e f} \\ P_{s e a l} & \\ r & \end{array}$




$\begin{array}{ll}t & \text { Time } \\ T & \text { Torque loss } \\ Z_{p} & \text { Number of teeth on pinion } \\ Z_{r} & \text { Number of teeth on ring gear } \\ v & \text { Kinematic viscosity } \\ \Omega_{p} & \text { Pinion speed (rpm) } \\ \Omega_{r} & \text { Ring gear speed (rpm) } \\ \phi & \text { Pinion position angle (deg) } \\ \rho & \text { Density }\end{array}$

$\underline{\text { Subscript }}$

$p$

$r$ $\underline{\text { Definition }}$

Pinion

Ring gear 


\section{CHAPTER 1}

\section{INTRODUCTION}

\subsection{Background and Motivation}

Federal and state carbon emissions and efficiency requirements on passenger vehicles and heavy trucks are becoming increasingly stringent. Higher fuel prices also bring the fuel mileage of these vehicles upfront as a major marketable feature. These requirements put every component of the powertrain under great scrutiny in terms of their power transmission efficiency. Since there is not a single power loss source that dominates the overall transmission efficiency, every source must be examined and minimized if possible, regardless how low they are, such that desired increases in efficiency can be achieved.

Reduction of power losses in a transmission provides several benefits other than increasing the fuel economy of the vehicle. Gear and bearing contacts produce high temperatures. Lower power losses equate to less heat generation in the gearbox, which improves the contact fatigue and scuffing performance of the gears and bearings. In 
relation to this, the capacity and extent of any lubrication system is also dictated by the amount of heat that must be removed from these critical contact zones. As such, design and durability of transmission systems are also impacted by their efficiency.

Increasing the overall efficiency of a transmission requires careful examination of all sources of power loss within the system. Focusing on the geared components of the transmission, the primary sources of power loss can be broken down into two categories [1], load-dependent (mechanical) power losses and load-independent (spin) power losses as shown in Figure 1.1. Mechanical power losses in gears primarily consist of frictional gear tooth interactions at the gear mesh, where the two primary modes of power loss are the rolling friction associated with the formation of an elastohydrodynamic (EHL) fluid film between meshing teeth and sliding friction associated with combined sliding-rolling velocities, normal forces, contact curvatures, and surface roughnesses. Alternatively, spin power losses in gears are associated with the interactions of the gear bodies with fluid medium around them. Two main components of gear spin power losses are:

(i) drag losses associated with churning of the oil or windage of air (or an oil-air mixture) along the side faces and periphery of gears, and

(ii) pocketing losses associated with pumping of fluid medium from pockets formed between adjacent teeth at the gear mesh interface.

Each component of power loss is associated with certain operating conditions. Mechanical losses are most significant at high-load, low-speed conditions when the fluid 


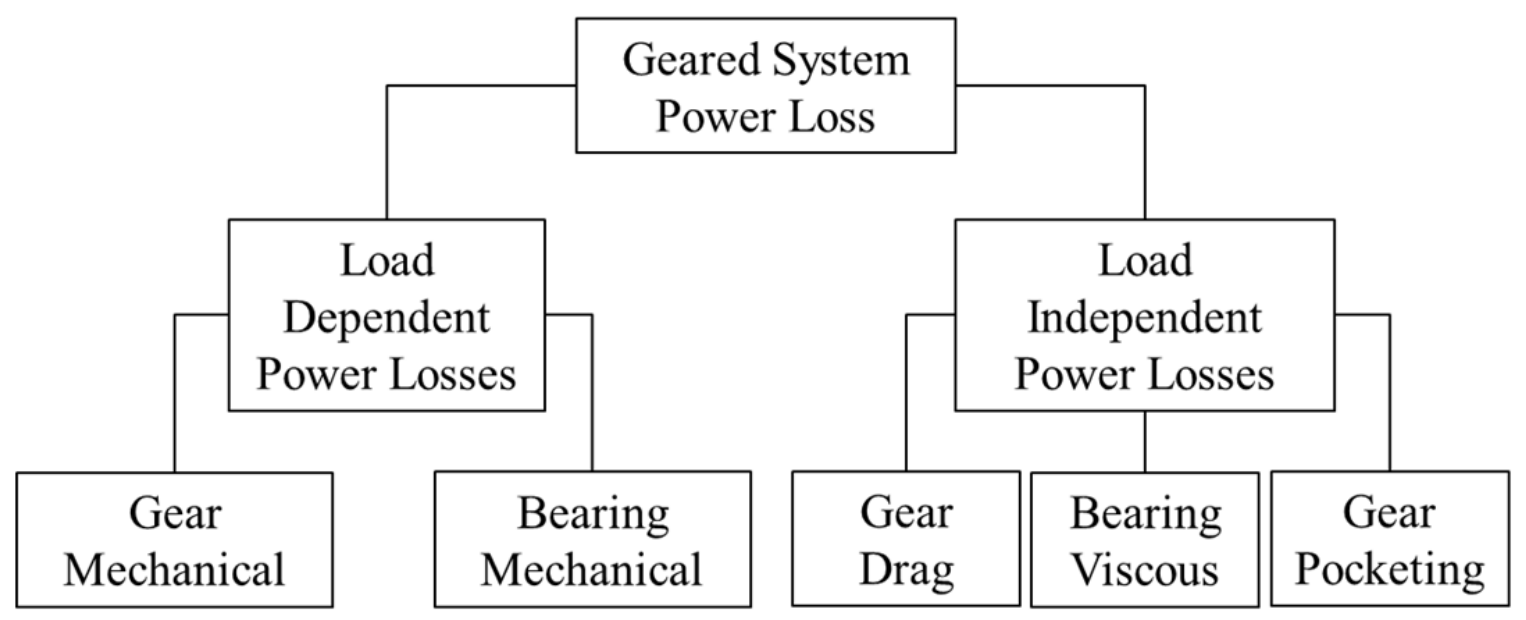

Figure 1.1 Main components of power losses of a geared system [1]. 
film formed at the contact interfaces is the least favorable, causing significant friction. In a vehicle, operation at low gear ratios produces such conditions. However, EPA cycles used to evaluate the fuel economy of a vehicle are dominated by high-speed (highway) driving conditions when spin losses are rather significant. A recent experimental study on the power losses of a six-speed manual transmission [2-5] showed that mechanical losses constitute the bulk of total loss in first gear operation, while as high as $90 \%$ of the total loss is spin loss when the transmission is operated in its top gear ratio.

Automotive transmissions mostly incorporate dip-lubrication techniques where the gears are partially immersed in lubricant and the motion of the gears supplies the circulation necessary to lubricate gear meshes and bearings. The energy required by the system to rotate the partially immersed gears and bearings is referred to as churning loss and is the primary focus of this study. A tightly-controlled experimental set-up and test procedure will be developed to investigate spin power losses of a helical gear pair of an actual transmission within wide ranges of speed, mesh configuration, oil level, and oil temperature. The space around the gears will also be altered to quantify any influences of enclosures and circumferential shrouds on spin losses of the gear pair. It is hoped that the database formed in this study will be instrumental in future modeling efforts in terms of the selection of assumptions employed, as well as validation of predictions. 


\subsection{Literature Review}

Most of the published work on gear efficiency has been on the measurement or prediction of mechanical losses. Spin losses are sought in these studies for the sole purpose of separating the mechanical losses from the total losses. Since the mechanisms and techniques to reduce mechanical losses are completely different from those for spin losses, these mechanical loss studies will not be reviewed here. A representative review of gear mechanical power losses can be found in recent publications [6-8].

The studies that focus on load-independent losses can be classified into those that investigate windage power losses and those investigating churning power losses. Windage and churning are not mutually exclusive, but are often studied separately by making assumptions based on operating conditions.

Windage power losses have the potential of being the dominant component of total power loss spectrum when operating conditions involve high pitch-line velocities, relatively low loads, and minimal lubricant [8]. Early fluid mechanics studies on cylindrical rotating disks have been viewed relevant to the gear windage problem. In one such study, Daily and Nece [9] studied the formation of the boundary layer along the surfaces of a rotating disk and proposed several flow regimes of the laminar and turbulent type. Mann and Marston [10] investigated the effects of windage on bladed disks and added the aspect ratio between the blade height and disk thickness. They found that generous clearances around a rotating disk with aspect ratios approaching unity can have

significant windage power loss components. Akin and Mross [11] further investigated 
the flow trajectories around gear teeth subjected to jet lubrication. They proposed that vapor flows axially in and out of gear teeth, which they called end windage, as well as related jet nozzle pressure to lubricant impingement depth. Dawson [12] ran experiments using toothed wheels and disks and found that gear teeth have a significant impact on windage losses, i.e. modeling gears as solid cylindrical disk might cause significant errors. He also found that a larger helix angle of the gear tooth lowered windage power loss. Lord [13] expanded on Dawson's experiments by adding several helical gear measurements. This study concluded that a greater helix angle translates into a greater axial component of flow, thus having less influence on torque loss.

Al-Shibl et al [14] used computational fluid dynamics (CFD) to predict windage loss of an enclosed spur gear, which showed a significant portion of windage power loss, could be attributed to pressure losses near the top lands of the gear teeth and found that adding a chamfer to the tooth tips can help reduce power loss. Handschuh and Hurrell [15] expanded the database for housing effects on windage losses using large diameter spur gears operating at extremely high pitch-line velocities and concluded that small radial and axial clearances had the greatest reduction on windage power loss, oil fling-off temperature, and static gearbox pressure. Hill et al [16] used CFD to model the gearbox used by Handschuh to validate the experimental results, as well as shed light on fluid flows around gear teeth. Seetharaman and Kahraman [17] proposed a fluid mechanics based model to predict power losses using compressible flow formulations for windage power loss along with incompressible flow formulations for churning power loss in dip 
lubricated systems [18]. They compared their model to experiments to establish their accuracy [19].

While interest in churning power losses of gearboxes is high, literature on such losses is rather sparse. Most of the earlier studies focused on rotating disks, where the complexity of the fluid flow around gear teeth is removed. Only a few newer studies have included the gear teeth on the rotating member(s). Early work by Terekhov [20] stressed that churning losses depend largely on flow regime and not as much on tooth geometry and lubricant viscosity. Boness [21] later noted using rotating disks that churning losses increase with increasing Reynolds number. Luke and Olver [22] pointed to certain discrepancies between the results of Terekhov and Boness and generated data to describe some of them. Similarly, Petry-Johnson [23] and Moorhead [24] expanded the churning experimental database using a back-to-back closed loop gear test rig to test the efficiency of spur and helical gears with varying module and surface finishes under jet and dip-lubricated conditions. More recently, experiments carried out by Changenet and Velex $[25,26]$ examined the influence of housing dimensions in the vicinity of a single rotating gear pair on churning losses. They noted under conditions where mating gears were pulling lubricant into mesh that the total churning loss was not simply the sum of the churning losses of the individual gears.

The difference between the sum of the power losses of two independently rotating gears and the power loss of the gear pair formed by these gears is the pocketing losses taking place at the gear mesh. Pechersky and Wittbrodt [27] developed a pocketing 
model for meshing spur gear teeth in an attempt to predict pressures and velocities. The spin loss model of Seetharaman and Kahraman [18] included a pocketing model as well to suggest that up to $90 \%$ of windage losses could be pocketing related. Diab et al [28] expanded on these to include helical gears. In order to fully define the pressure and velocity distribution for compressible flow they used conservation of energy in each pocket, which might be erroneous because since there are power losses in the system. Most recently, Talbot [1] and Talbot et al [29] developed a generic fluid dynamics based pocketing model capable of predicting pocketing power losses in helical gears as well as other types of gears. These studies found that, at sufficiently high pitch line velocities, the rapid expulsion of the fluid medium from the meshing zone causes a significant portion of the total power loss.

The goal of this study is to illuminate and quantify components of power loss that have not yet been examined as well as to confirm the findings of earlier experimental works. The experimental investigation presented in the proceeding chapters will attempt to add to past studies and provide additional validation data for the ongoing modeling efforts on gear spin losses.

\subsection{Scope and Objectives}

As the literature review above indicates, the majority of experimental studies on gear efficiency focused on mechanical power losses with no attention given to spin power losses. The few experimental studies dedicated to studying spin power losses were limited to a few housing configurations, and circumferential mesh locations, if any. The 
main purpose of this study is to establish an extensive database through conducting tightly-controlled experiments in an effort to isolate and determine the influence of possible components of spin power losses in geared systems. With this aim, specific technical objectives of this study are as follows:

(i) Design, fabricate and run tests with a new gearbox suitable for gear pair spin loss evaluations.

(ii) Incorporate the test gearbox with a high-speed test bed and its instrumentation designed to measure key efficiency related parameters such as torque loss and temperatures.

(iii) Use the experimental set-up to investigate the influence of (a) rotational speed, (b) lubricant temperature, (c) lubricant type, and (d) static oil level on drag churning losses of a single gear.

(iv) Perform experiments with a cylindrical disk, a spur gear, and a helical gear of the same outside diameter to determine the influence of gear teeth and helix angle on drag power losses of a gear pair.

(v) Use the experimental set-up to investigate the influence of (a) rotational speed, (b) lubricant temperature, (c) lubricant type, (d) static oil level and (e) circumferential mesh location on spin (drag plus pocketing) losses of a helical gear pair. Compare these to single gear results of objective (iii) to determine the pocketing component of spin power loss. 
(vi) Design and procure enclosures and shrouds, and perform tests with such surroundings to determine their influence on spin losses of a single gear and a gear pair.

\subsection{Thesis Outline}

Chapter 2 provides a detailed description of the design of the test gearbox, as well as details about the test machine that was used with it. The primary variables of interest to the designer, as well as the implementation and control of those variables are discussed. The testing procedure that was followed and the test specimens are specified in detail. Finally, a test matrix explaining all the parameter studies is presented. Chapter 3 walks through all the sub-studies in detail and presents detailed results of each study. Chapter 4 discusses the results and provides sets of conclusions and recommendations for future work. 


\section{CHAPTER 2}

\section{EXPERIMENTAL TEST METHODOLOGY}

\subsection{Design and Development of the Test Gearbox}

The objectives for this study cover a broad range of testing conditions and gearbox configurations. Accordingly, a test gearbox was required to handle many different operating conditions and gearbox configurations. Primary requirements from the test gearbox are listed below:

(i) The test gearbox must be able to accommodate a family of gear pairs of different gear ratios, but the same center distance.

(ii) The test gearbox should accommodate a single gear (spur or helical) or a cylindrical disk that is placed on the input shaft such that the impact of spur or helical gear teeth on drag losses of a single rotating gear can be quantified.

(iii) The bearings of the input and output shafts must be identical such that bearing viscous losses of both shafts can be separated from gear-specific losses. 
(iv) The gearbox must be rotatable to different positions about the input shaft axis such that various circumferential positions of the gear mesh can be achieved.

(v) The gears must be supported in an overhung configuration and the front and top covers of the gearbox must be transparent for viewing of the fluid flow during tests. This transparent arrangement is required for verifying the static oil levels as well.

(vi) The interior of the gearbox must have the necessary details to accommodate fillers to change the space around the gears through the use of enclosures.

(vii) The gearbox in all of its above configurations must be compatible with an existing high-speed test bed and its measurement and lubrication systems.

As the first step in the design process, a family of automotive transmission final drive gear pairs was chosen by the sponsor as the example gear sets. This family consists of three gear pairs of ratios $n=4.563,4.250$ and 3.941, all operating at the same center distance of $137.57 \mathrm{~mm}$. Table 2.1 lists basic design parameters of these three gear pairs and Figure 2.1 shows a complete set of the gear pair specimens. Following the automotive terminology, the smaller gear that is an integral part of its shaft will be called the pinion and the larger gear that is typically bolted to the differential assembly of the transmission will be named the ring gear. As the ratios are quite close to each other, the 
Table 2.1 Basic design parameters of final drive gear pairs used in this study.

\begin{tabular}{|c|c|c|c|c|c|c|}
\hline \multirow[b]{2}{*}{ Parameter } & \multicolumn{2}{|c|}{ Gear Ratio \#1 } & \multicolumn{2}{|c|}{ Gear Ratio \#2 } & \multicolumn{2}{|c|}{ Gear Ratio \#3 } \\
\hline & $\begin{array}{l}\text { Ring } \\
\text { Gear }\end{array}$ & Pinion & $\begin{array}{l}\text { Ring } \\
\text { Gear }\end{array}$ & Pinion & $\begin{array}{l}\text { Ring } \\
\text { Gear }\end{array}$ & Pinion \\
\hline $\begin{array}{c}\text { Number of } \\
\text { Teeth }\end{array}$ & 73 & 16 & 68 & 16 & 67 & 17 \\
\hline $\begin{array}{c}\text { Final Drive } \\
\text { Ratio }\end{array}$ & \multicolumn{2}{|c|}{4.563} & \multicolumn{2}{|c|}{4.250} & \multicolumn{2}{|c|}{3.941} \\
\hline $\begin{array}{c}\text { Normal } \\
\text { Module }[\mathrm{mm}]\end{array}$ & \multicolumn{2}{|c|}{2.58} & \multicolumn{2}{|c|}{2.73} & \multicolumn{2}{|c|}{2.73} \\
\hline Pressure Angle & \multicolumn{2}{|c|}{$21.0^{\circ}$} & \multicolumn{2}{|c|}{$21.0^{\circ}$} & \multicolumn{2}{|c|}{$21.0^{\circ}$} \\
\hline Helix Angle & \multicolumn{2}{|c|}{$33.5^{\circ}$} & \multicolumn{2}{|c|}{$33.5^{\circ}$} & \multicolumn{2}{|c|}{$33.5^{\circ}$} \\
\hline $\begin{array}{c}\text { Center } \\
\text { Distance }[\mathrm{mm}]\end{array}$ & \multicolumn{2}{|c|}{137.57} & \multicolumn{2}{|c|}{137.57} & \multicolumn{2}{|c|}{137.57} \\
\hline $\begin{array}{l}\text { Face Width } \\
{[\mathrm{mm}]}\end{array}$ & 28.5 & 34.55 & 28.5 & 34.55 & 28.5 & 34.55 \\
\hline $\begin{array}{c}\text { Outside } \\
\text { Diameter } \\
{[\mathrm{mm}]}\end{array}$ & 230.10 & 58.55 & 228.05 & 62.09 & 224.67 & 65.44 \\
\hline
\end{tabular}



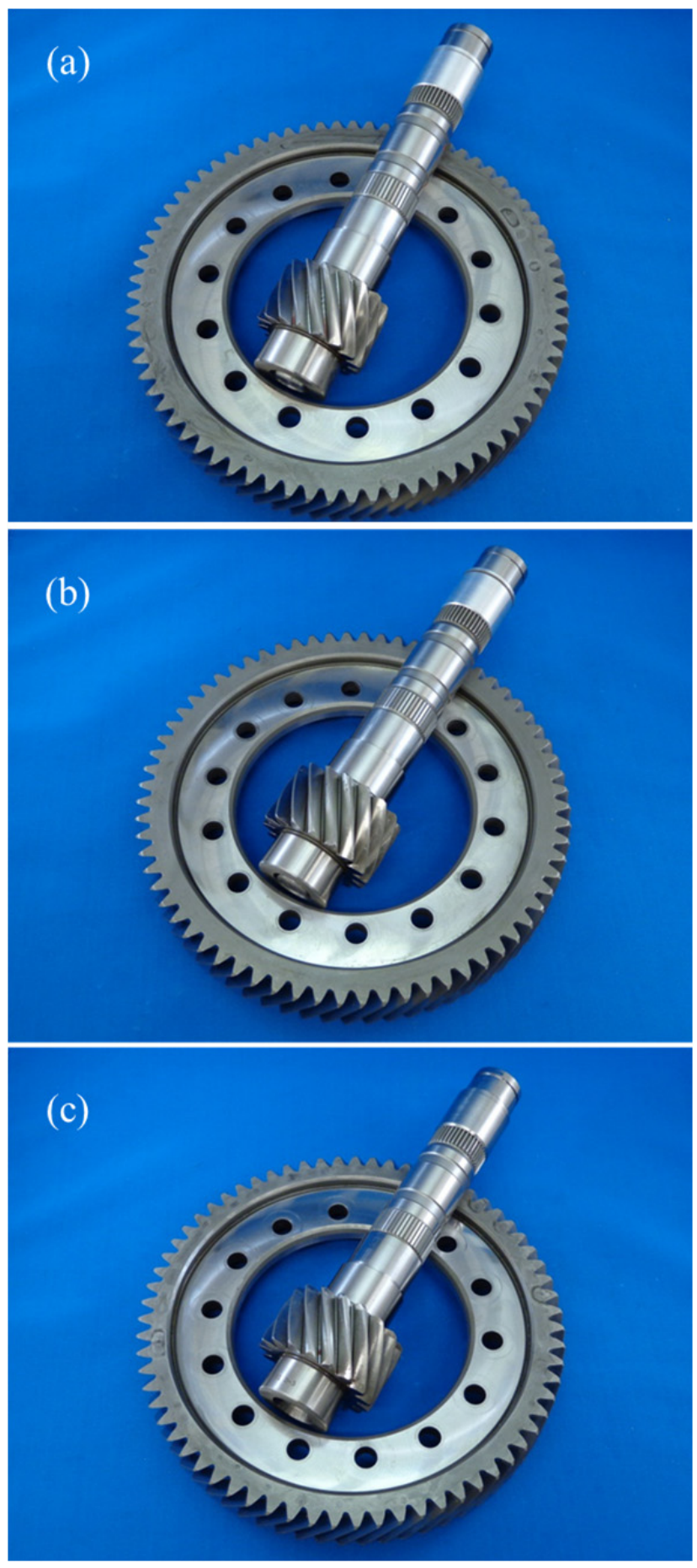

Figure 2.1 Pictures of (a) gear pair \#1, (b) gear pair \#2, (c) gear pair \#3. 
only main difference amongst these gear pairs are the diameters of the gears as shown in Figure 2.2(a) for the pinions and Figure 2.2(b) for the ring gears. The basic blank and shaft details of each gear pair is such that all three can be accommodated in the same gearbox, hence satisfying requirement (i) above.

A three-dimensional solid model of the gearbox design is shown in Figure 2.3. Figure 2.4(a) provides a cross-sectional view of the same and Figure 2.5 shows a picture of the gearbox. Also shown in Figure 2.4(b) is the configuration with only the ring gear such that certain aspects of design requirement (ii) above can be met. In this case, the pinion shaft is removed from the gearbox and its bearing housing is plugged by a cylindrical flange. In this configuration, the torque loss measured can only be attributed to the ring gear drag losses and the viscous losses of the pair of input bearings.

The single-gear configuration shown in Figure 2.4(b) can be used to test a replacement spur gear or a solid disk in place of the helical ring gear such that the impact of ring gear teeth, as well as helix angle on gear drag can be quantified. For this purpose, an equivalent spur ring gear (at the same transverse geometry as the helical ring gear), and a solid blank (whose outside diameter is equal to the major diameter of the helical gear) were designed and procured. Figure 2.6 shows the helical, spur and solid blank equivalents of the ring gear, which were all designed to be mounted on the same input shaft in exactly the same way. This way, the gearbox design met requirement (ii) above satisfactorily. 

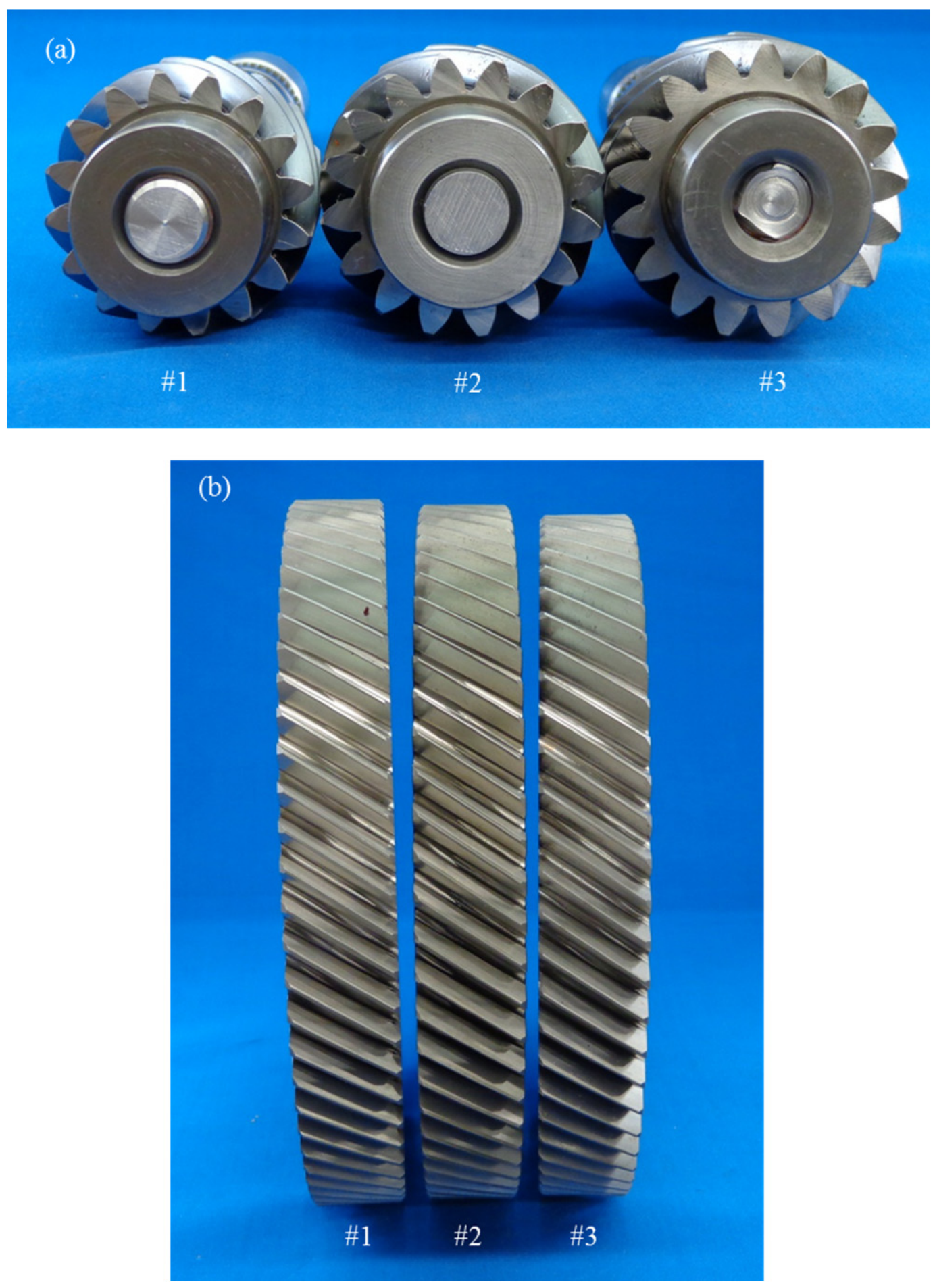

Figure 2.2 (a) Pinions and (b) ring gears of the test gear pairs. 


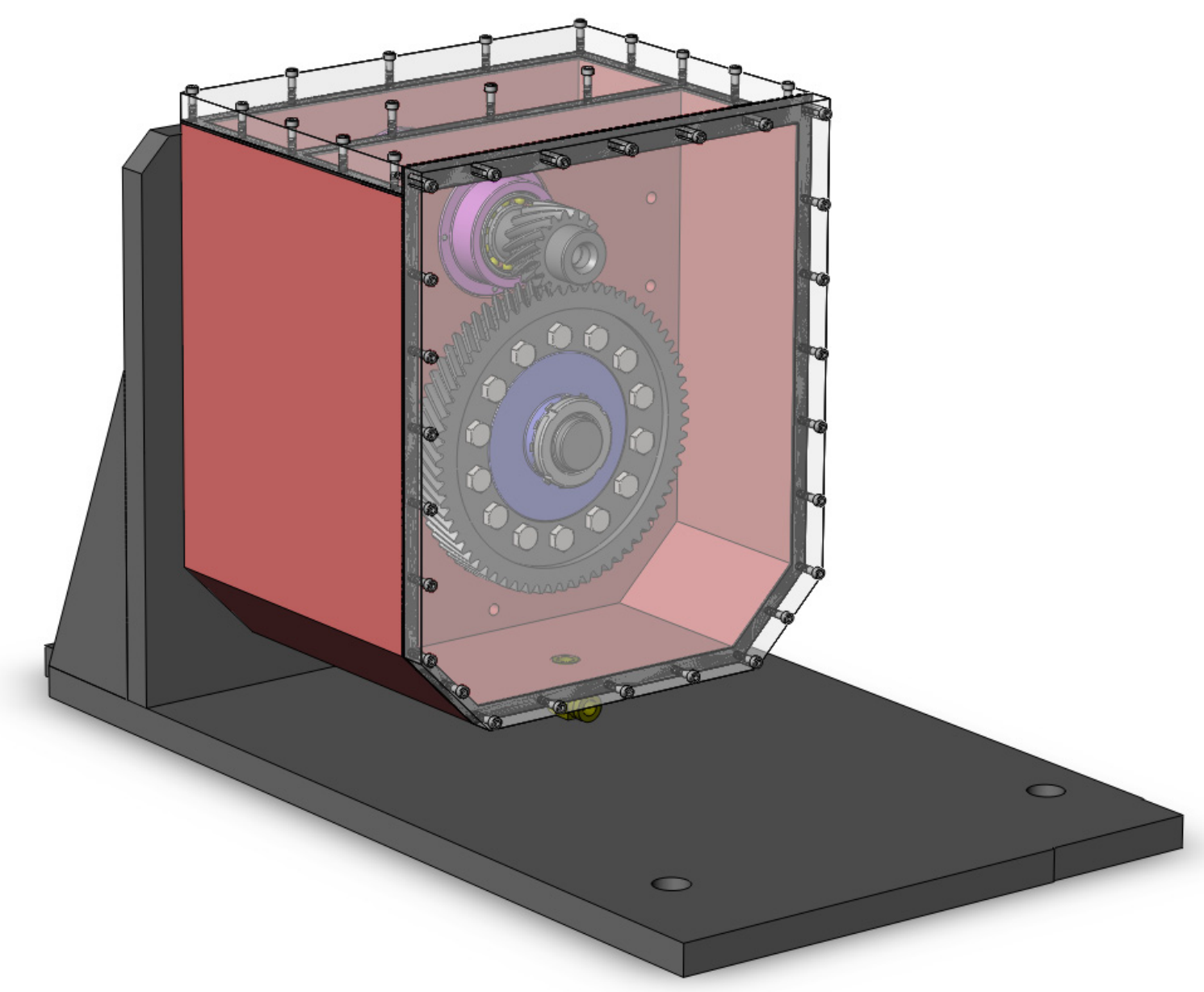

Figure 2.3 Solid model of the test gearbox used in this study. Side and top covers are made of polycarbonate material for viewing purposes. 


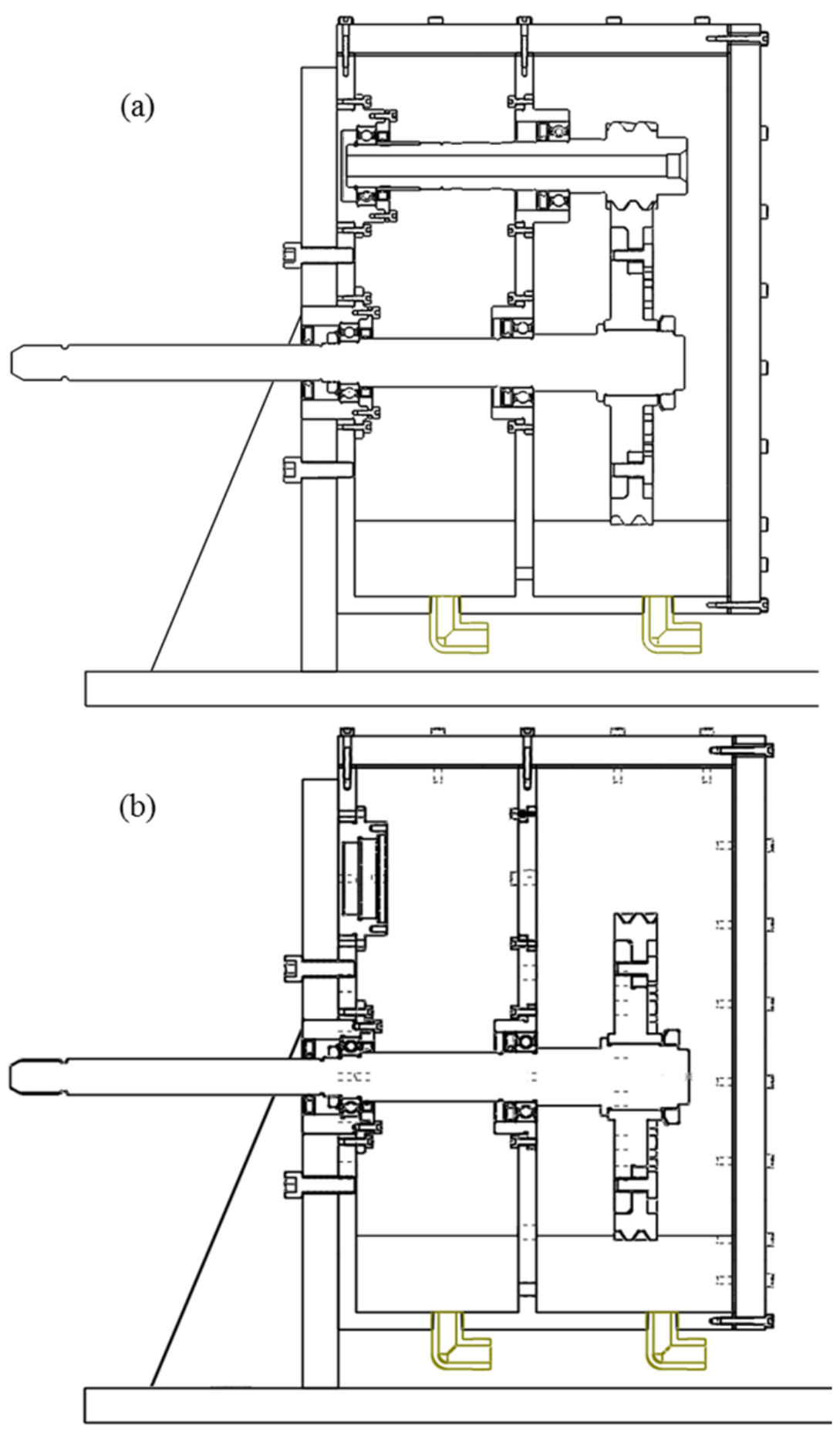

Figure 2.4 Cross-sectional drawings of the test gearbox (a) with both gears assembled, and (b) with only the ring gear assembled. 


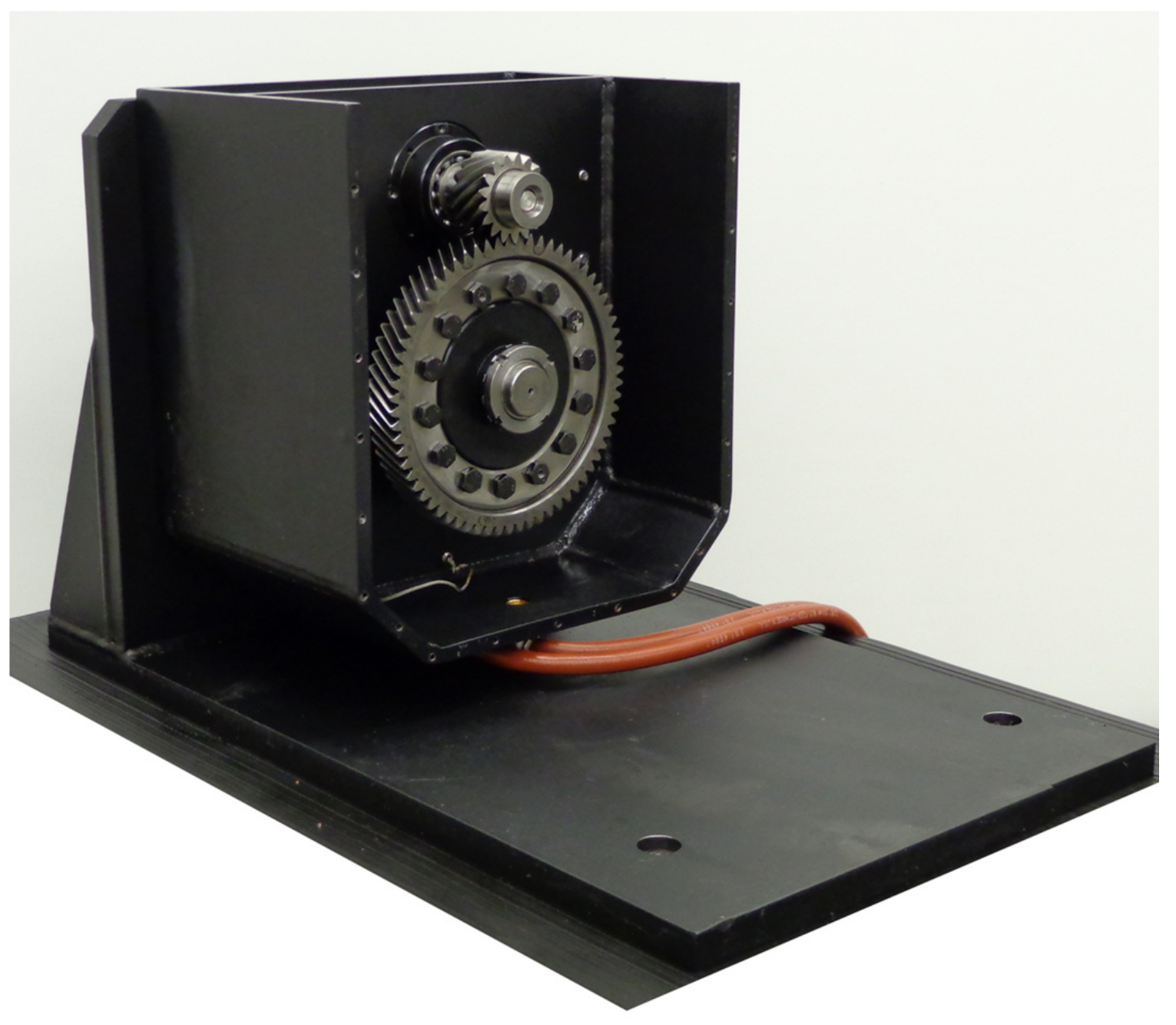

Figure $2.5 \mathrm{~A}$ picture of the assembled test gearbox. 

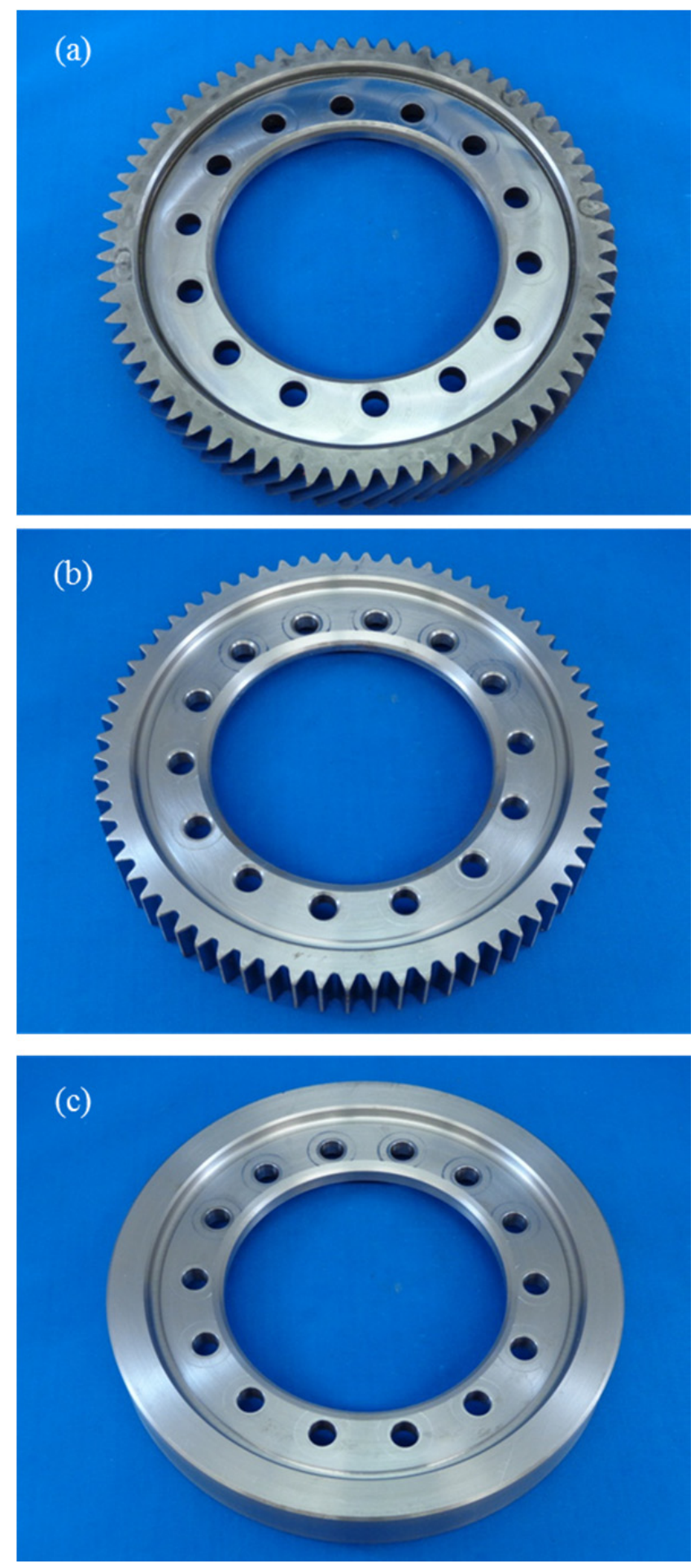

Figure 2.6 (a) Helical ring gear, (b) spur ring gear (c) ring gear blank with no teeth, and (d, e) side views comparing each variation. (continued) 
Figure 2.6 (continued)
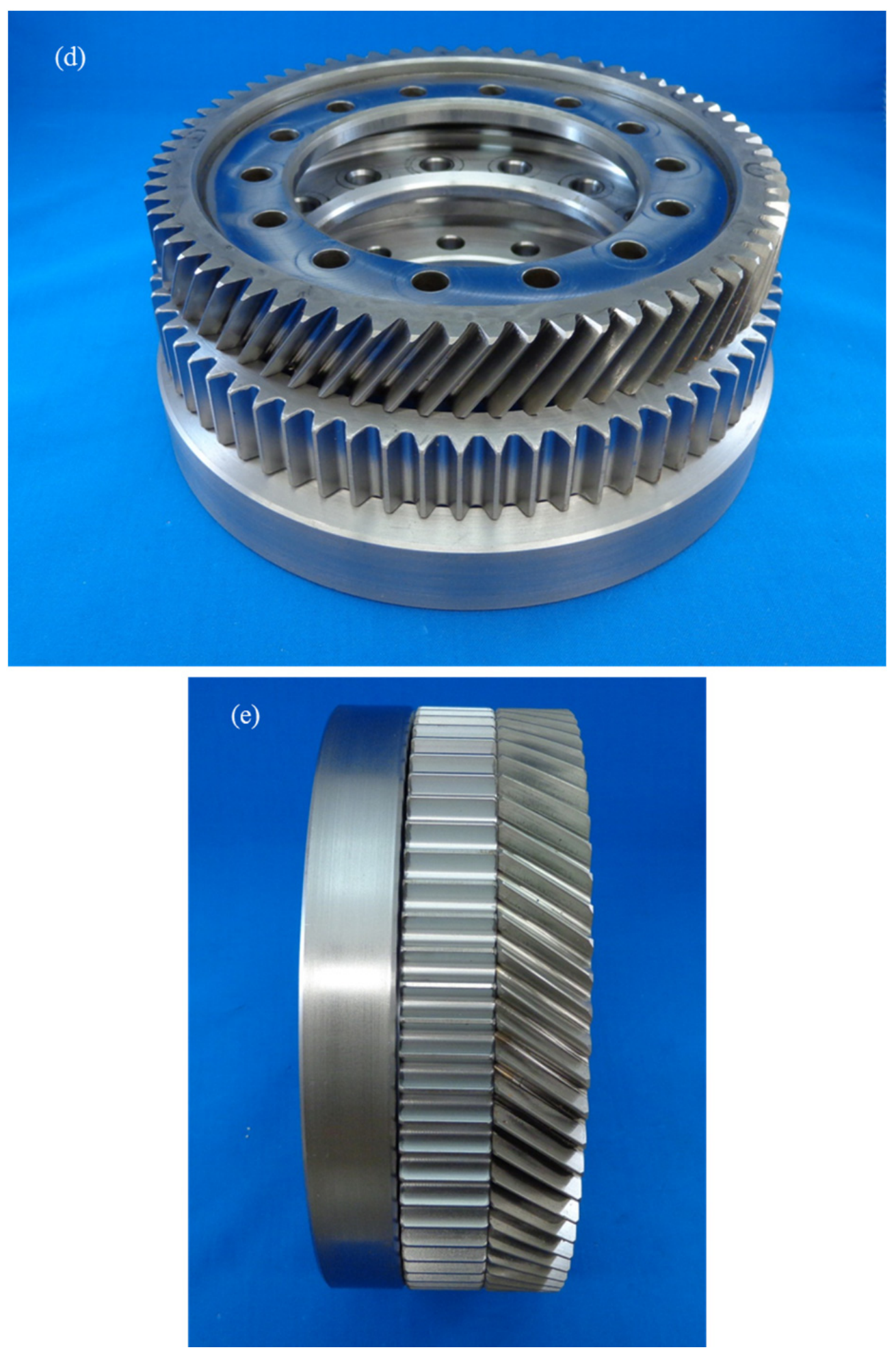
When operated in the single-shaft configuration of Figure 2.4(b) with no ring gear assembled on the input shaft, the measured torque losses represents primarily the viscous bearing losses and drag losses of the input shaft, the latter being negligible. This configuration will be used to determine viscous losses of the input shaft bearings. Deep groove ball bearings specified in Figure 2.7 were chosen here with no preload such that there is no variability introduced in the assembly. Each bearing was held by its own bearing housing. The bearing to the right in Figure 2.4(b), the one closer to the ring gear, was exposed to the gearbox on its right hand side to ensure that it is lubricated properly by the oil bath in the gearbox. Meanwhile, the other side of this bearing housing was sealed by using a radial shaft seal to prevent any leaking of the oil in the gearbox though this bearing. The other bearing to the left in Figure 2.4(b) was also contained within its own housing with at both sides of the bearings such that this bearing could be operated in its own oil bath.

Comparison of the bearings in both shafts in Figure 2.4(a) reveals that both sets of bearings are identical. The only difference is that the left bearing housing of the pinion shaft has one less seal. As losses associated with these types of seals are rather small, it can be assumed that the bearing viscous losses of the pinion and ring gear shafts must be equal, provided that they are rotated at the same speed. Since the ring gear shaft will be connected to the test machine to act as the input shaft for this gearbox, running the same test with configuration of Figure 2.4(b) without the ring gear establishes the bearing 


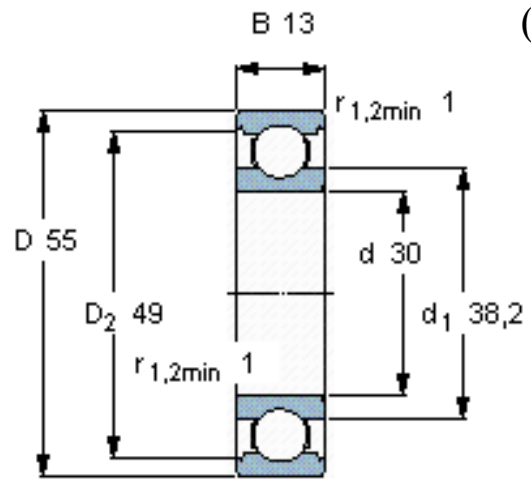

(a)
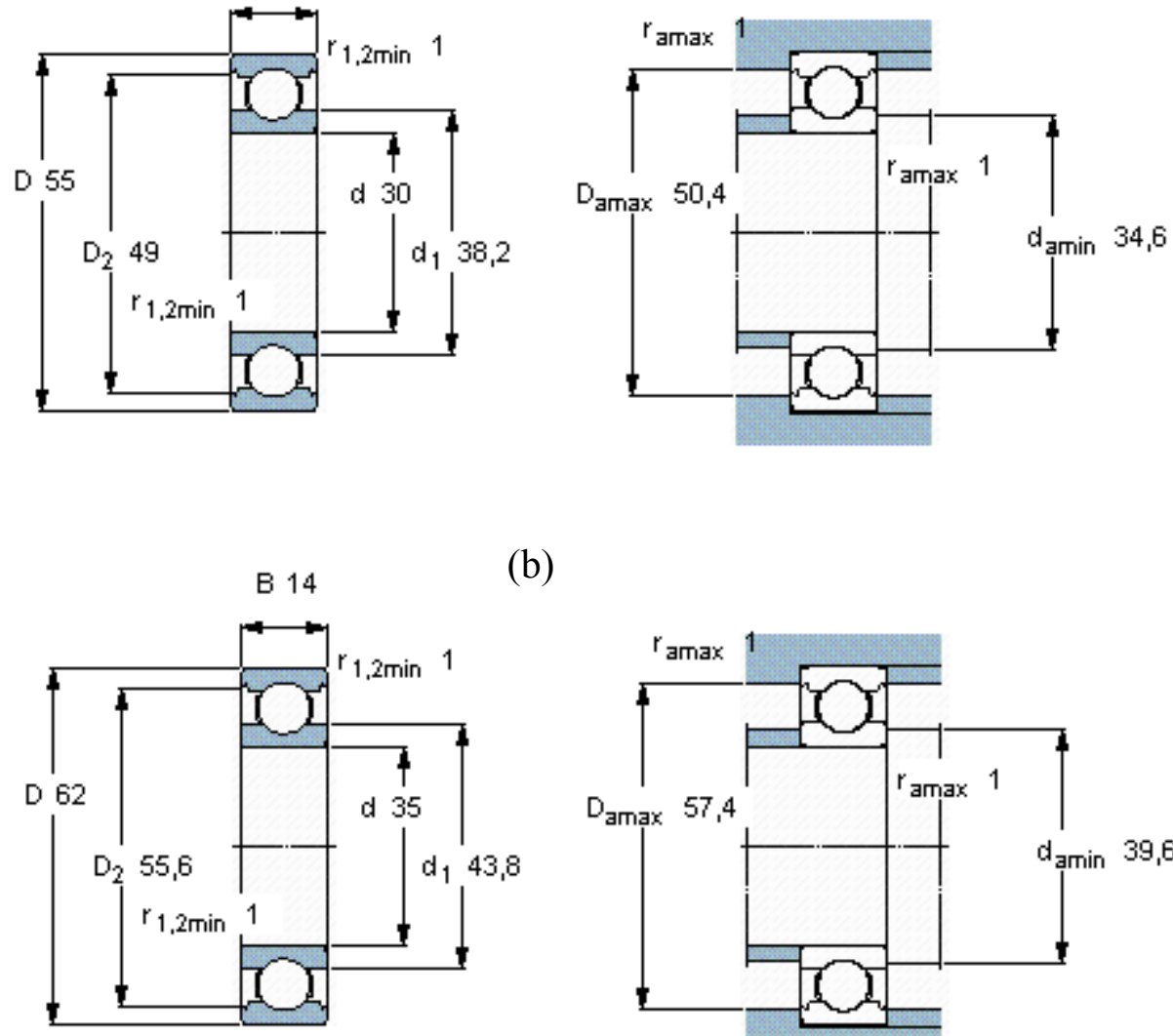

(b)

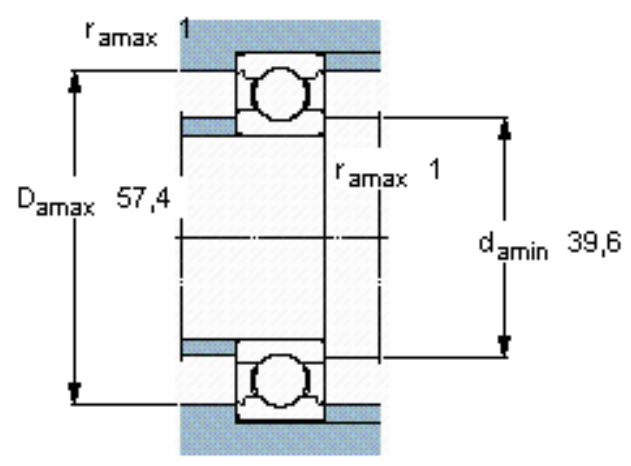

Figure 2.7 (a) Inboard deep-groove ball bearings (6006*) [36], and (b) outboard deepgroove ball bearings $\left(6007^{*}\right)$ [37] used to support both shafts of the gearbox. All dimensions are in $\mathrm{mm}$. 
losses of the ring gear shaft. With a given gear ratio of $n=Z_{r} / Z_{p}=\Omega_{p} / \Omega_{r}$ where $Z_{r}$ and $Z_{p}$ are the number of teeth of the ring gear and the pinion, the pinion bearings rotate at $\Omega_{p}=n \Omega_{r}$ when the ring gear shaft is rotating at $\Omega_{r}=\Omega_{i n}$. Given the equivalency of the bearings of both shafts, losses of the pinion shaft bearings can also be quantified by repeating a bearing viscous loss test at an elevated input speed of $\Omega_{i n}=n \Omega_{r}$. This satisfies requirement (iii) listed above.

Requirement (iv) above was that the circumferential position of the pinion relative to the ring gear must be variable. This was done by designing matching bolt patterns on the back of the gearbox and the T-shaped support structure. Different circumferential pinion positions were obtained by aligning different sets of holes and fastening the gearbox in those positions. Figure 2.8 shows the front view of the gearbox in two extreme pinion positions of $\phi=0^{\circ}$ and $\phi=180^{\circ}$, as well as the baseline vertical position of $\phi=90^{\circ}$ where the line formed by the pinion and ring centers is vertical (i.e. the pinion is on top of the ring). The gearbox design allowed the discrete pinion position angles of $\phi=0^{\circ}, 30^{\circ}, 45^{\circ}, 60^{\circ}, 90^{\circ}, 120^{\circ}, 135^{\circ}, 150^{\circ}$, and $180^{\circ}$, as shown schematically in Figure 2.9. Here, no symmetry exists about $\theta=90^{\circ}$ (i.e. losses at $\phi=90^{\circ}+x^{\circ}$ and $\phi=90^{\circ}-x^{\circ}$ should differ) due to the rotational directions of the gears. As shown in Figure 2.9, when the pinion is at $\phi=180^{\circ}$, oil is pulled in to the mesh while the 
(a)

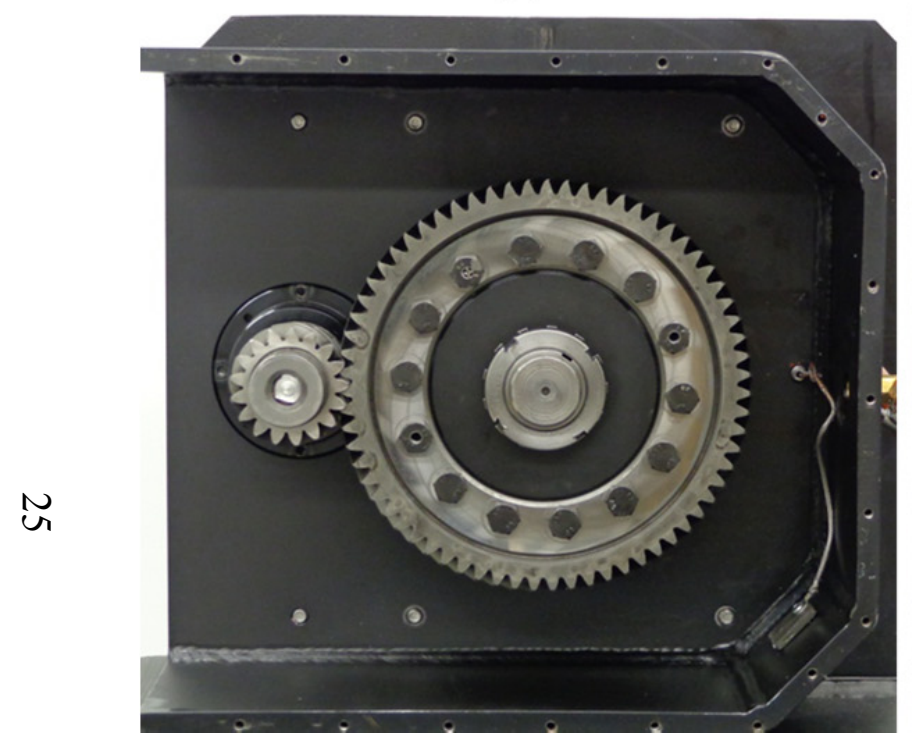

(b)

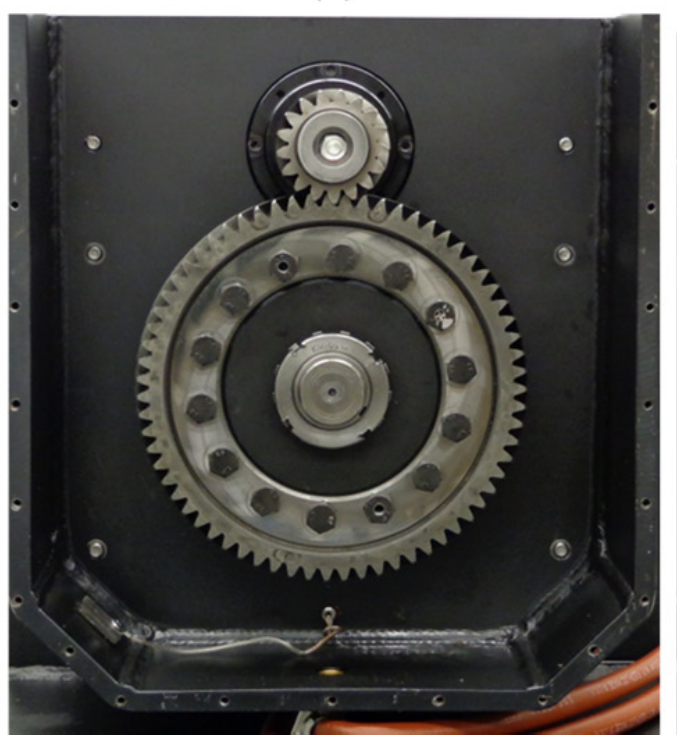

(c)

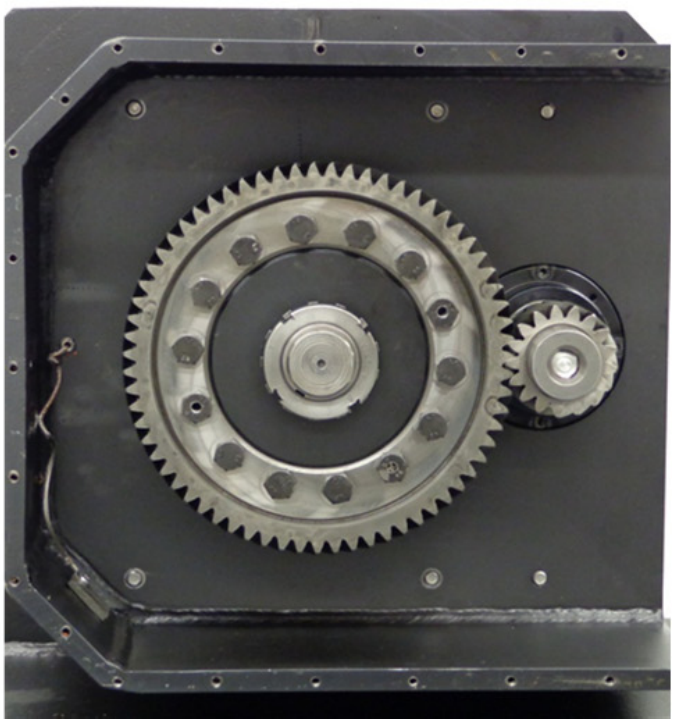

Figure 2.8 The views of the test gearbox in circumferential pinion positions of (a) $\phi=0^{\circ}$, (b) $\phi=90^{\circ}$, and (c) $\phi=180^{\circ}$. 


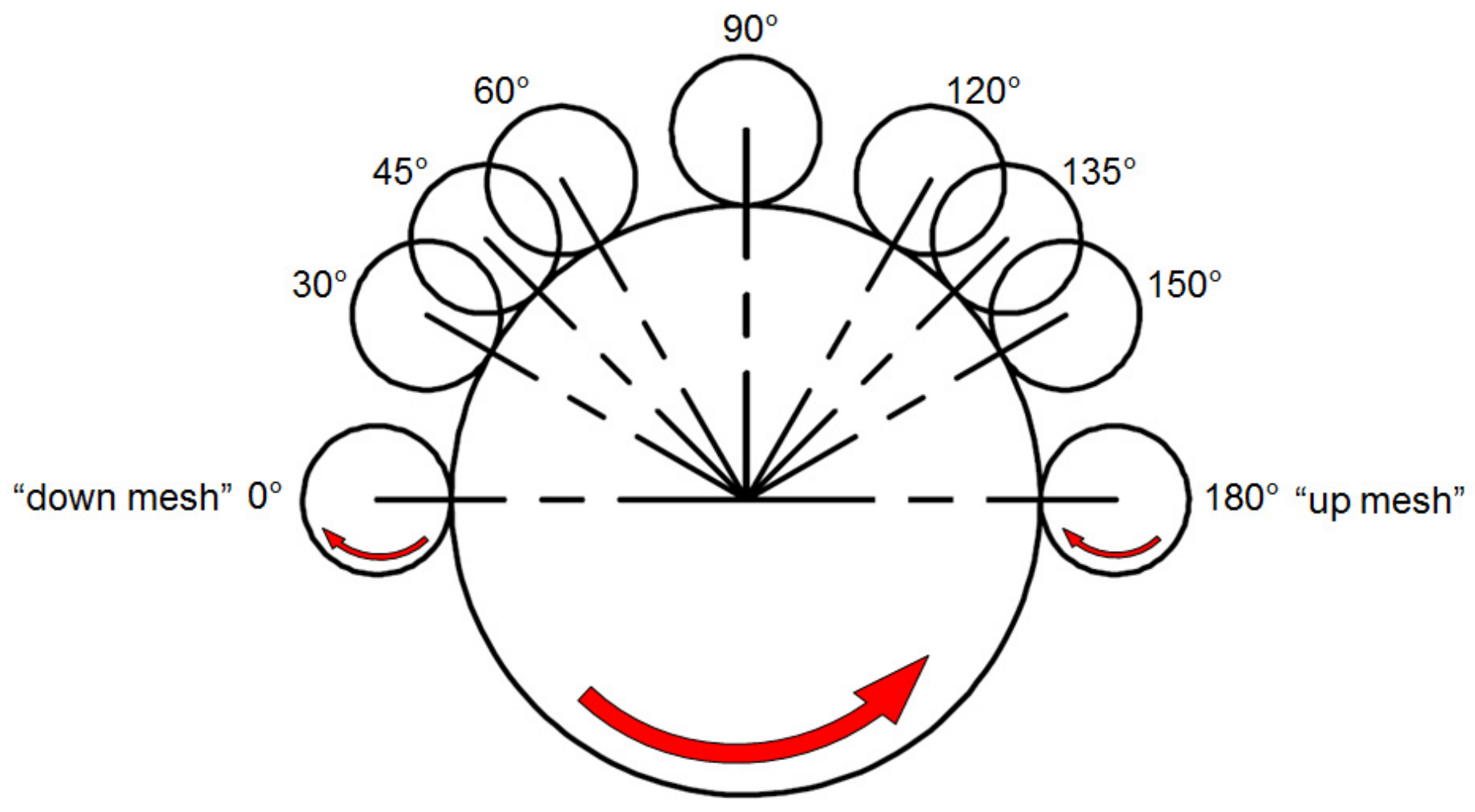

Figure 2.9 Circumferential pinion position angles $\phi$ achievable by the test gearbox. 
opposite is true at $\phi=0^{\circ}$. As such, the gearbox designed here is capable of testing such variations, in the process satisfying design requirement (iv) above.

While it is not possible to define an effective oil level under dynamic operating conditions, the corresponding static oil levels have been used to represent the degree of immersion of the ring gear. Petry-Johnson et al [6], Moorhead [24] and Seetharaman and Kahraman $[18,19]$ used a dimensionless static oil level parameter $\bar{h}=h / r$ where $h$ is height of the oil level measured from the bottom of the gear and $r$ is the outside radius of the gear, as illustrated in Figure 2.10(a). Figure 2.10(b) shows static oil levels at different $\bar{h}$ values. Here, $\bar{h}=0$ indicates the case when oil is not in contact with the gear. $\bar{h}=1.00$ when the gear is halfway immersed in oil while $\bar{h}=2.00$ when the static oil level is at the top of the gear such that entire gear is submerged in oil. Also marked in Figure 2.10(b) is when $\bar{h}=0.05$ representing a static oil level that is tangent to the root circle of the gear. The front and top covers of the gearbox shown in Figure 2.3 were made of $20 \mathrm{~mm}$ thick transparent polycarbonate such that desired $\bar{h}$ value can be set and verified conveniently. Furthermore, this allowed viewing of oil circulation during an efficiency test, in the process meeting design requirement (v) above.

In a companion study, Seetharaman and Kahraman [33] predicted that the distance between the axial and peripheral gap between the gear and its enclosure might impact spin losses significantly. In an attempt to study such effects and hence meet requirement (vi) above, sets of polycarbonate sheets were designed and fabricated to be 

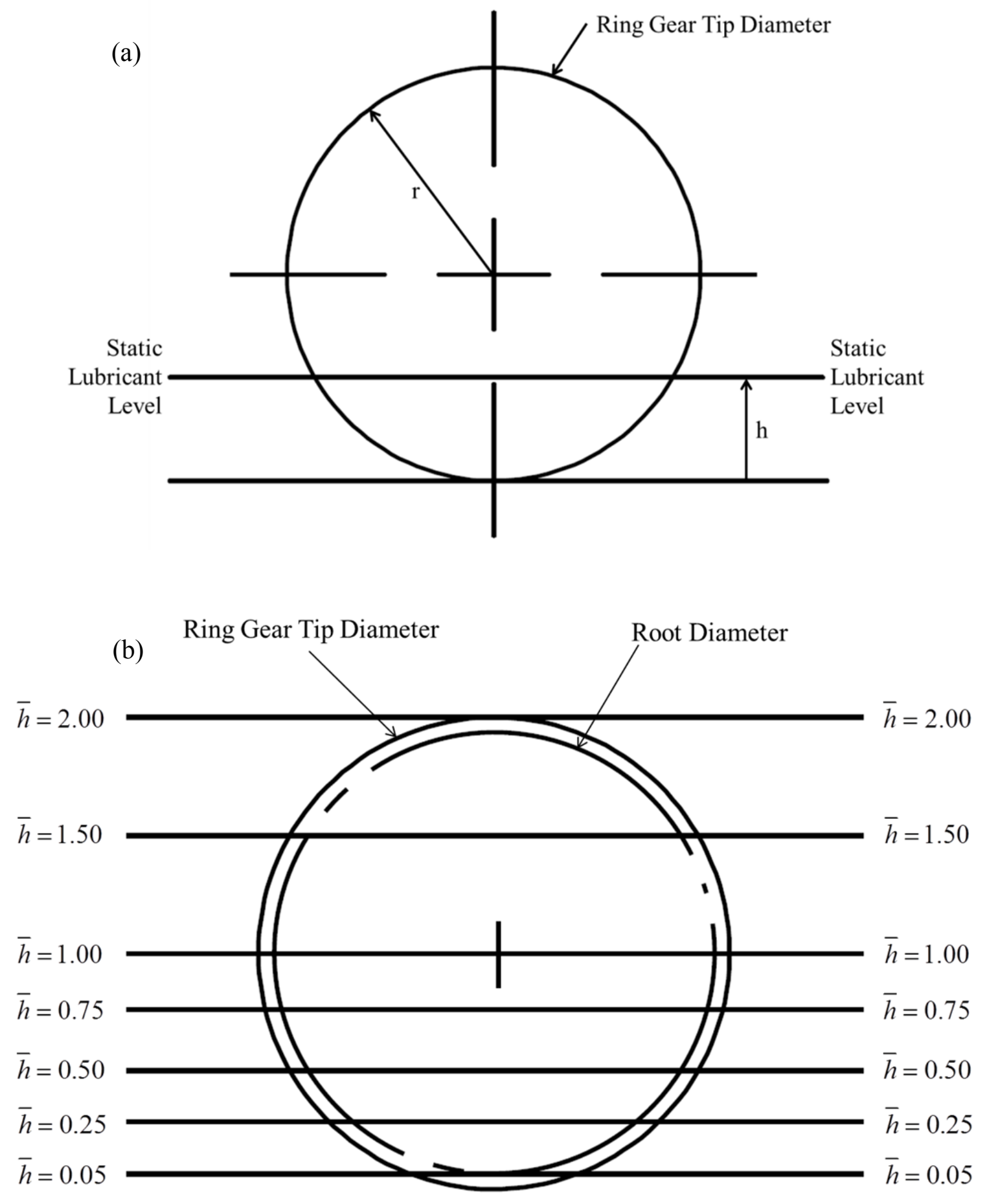

Figure 2.10 (a) Parameters defining of the static oil level parameter $\bar{h}$ and (b) static oil levels considered in this study. 
mounted in the gearbox to control the space around the gears. As shown in Figure 2.11, two parameters were defined as axial gap, $s$, and peripheral gap, $p$. Here, the $s$ dimension was considered to be the same on both sides of the gear such that one axial gap parameter is sufficient. The dimensions of the gearbox in Figure 2.3 and 2.5 were such that $s=50$ $\mathrm{mm}$ and $p \geq 50 \mathrm{~mm}$ without any of these polycarbonate fillers assembled, representing "almost infinite" gearbox dimensions. Sets of fillers allowed designed gap values of $s=1,5$ and $12 \mathrm{~mm}$ as well as $p=5$ and $12 \mathrm{~mm}$. The gearbox with some of these fillers assembled is shown in Figure 2.12. It is noted here that the axial and peripheral enclosures did not surround the gear to allow some volume within the gearbox on top for oil to accumulate and then return the gear-enclosure interface. As such, enclosures shown in Figure 2.12 cover the ring gear up to the static oil level of $\bar{h}=1.93$, leaving a $90^{\circ}$ segment on top out of enclosures. Each polycarbonate plate was designed to have a number of holes that were used to locate the plate within the gearbox. Layers of such plates were clamped together to occupy all of the space other than the desired gaps defined by parameters $s$ and $p$, in the process meeting design requirement (vi) above.

\subsection{Test Machine}

As stated earlier, a high-speed test machine was used as a test bed in various earlier efficiency experiments $[6,23,24,30,34]$. Requirement (vii) in the previous section indicated that the gearbox designed for this study must comply with this existing 


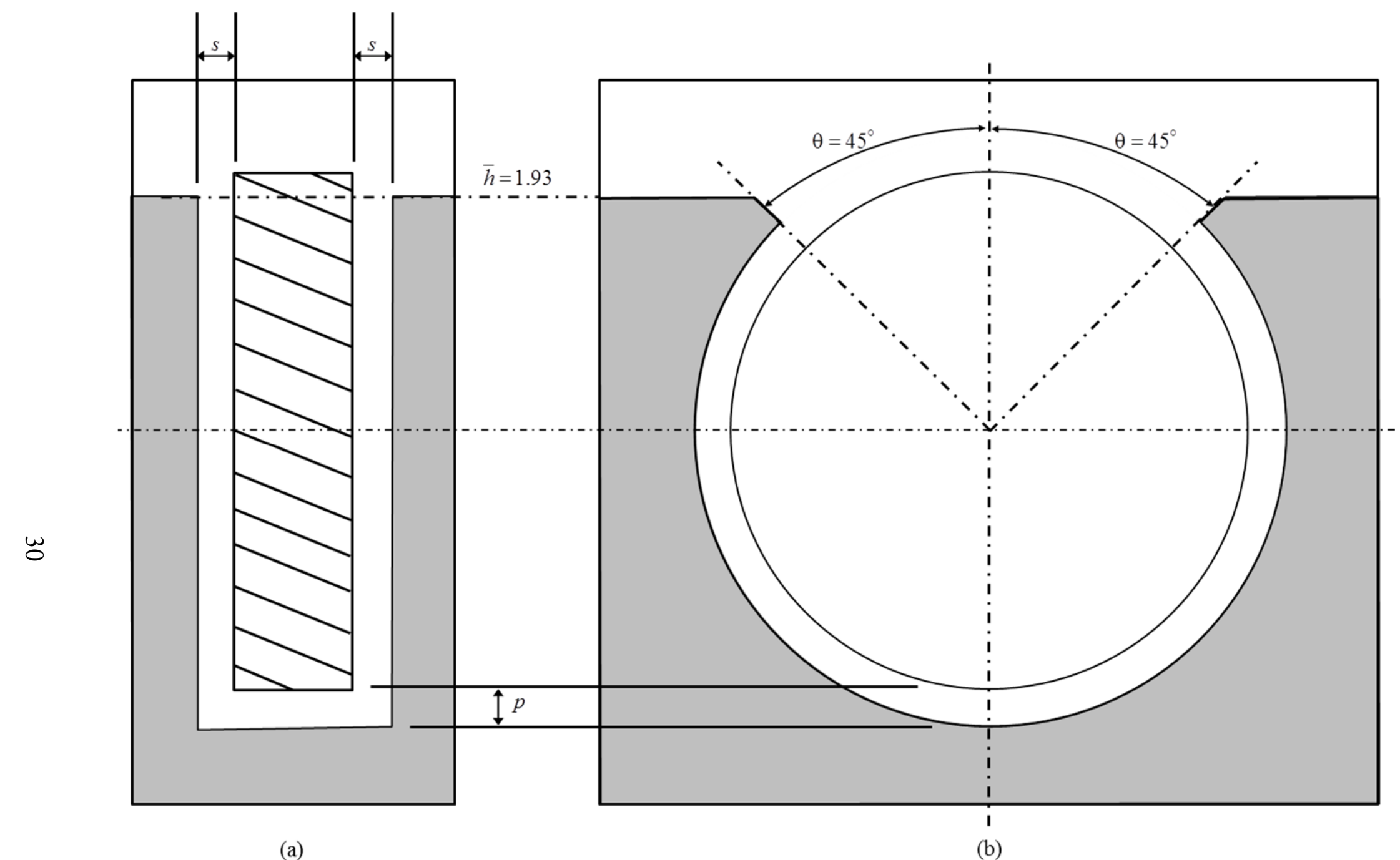

Figure 2.11 (a) Side and (b) frontal view schematics of the gearbox defining the enclosure configurations used in this study. 
(a)

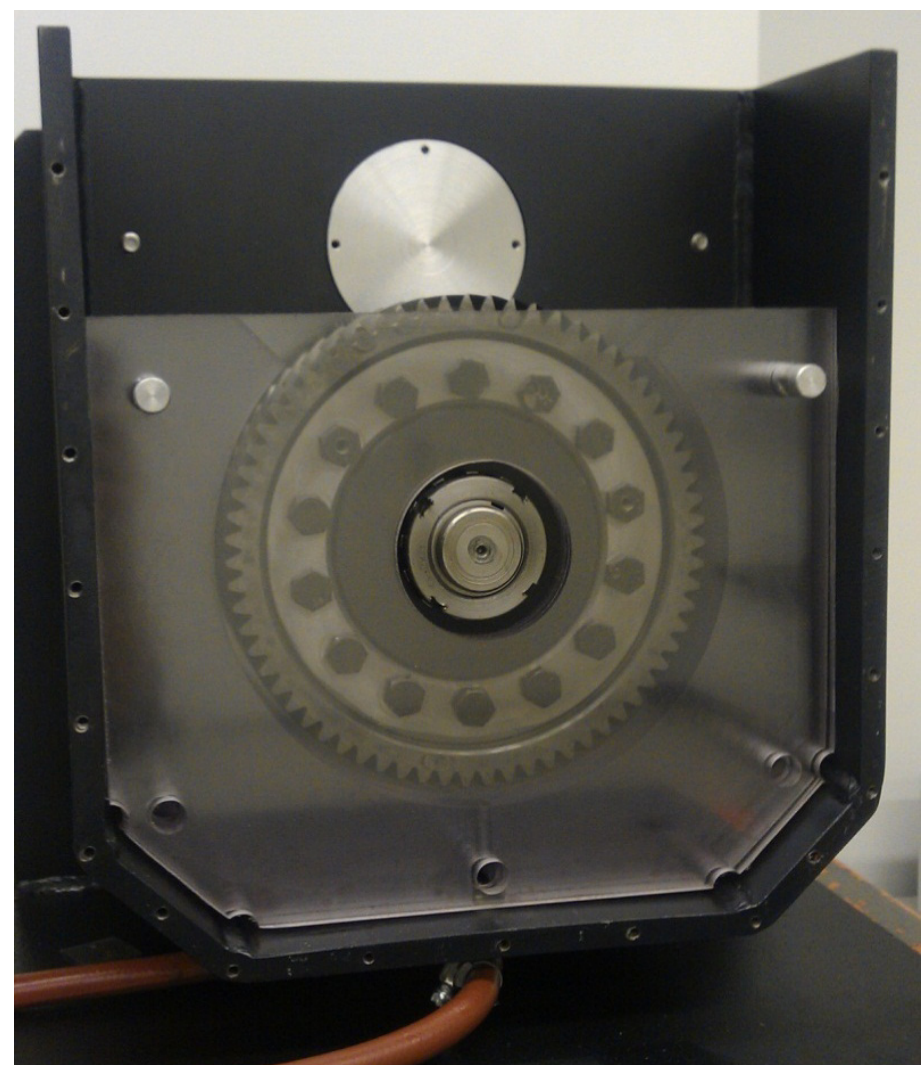

(b)

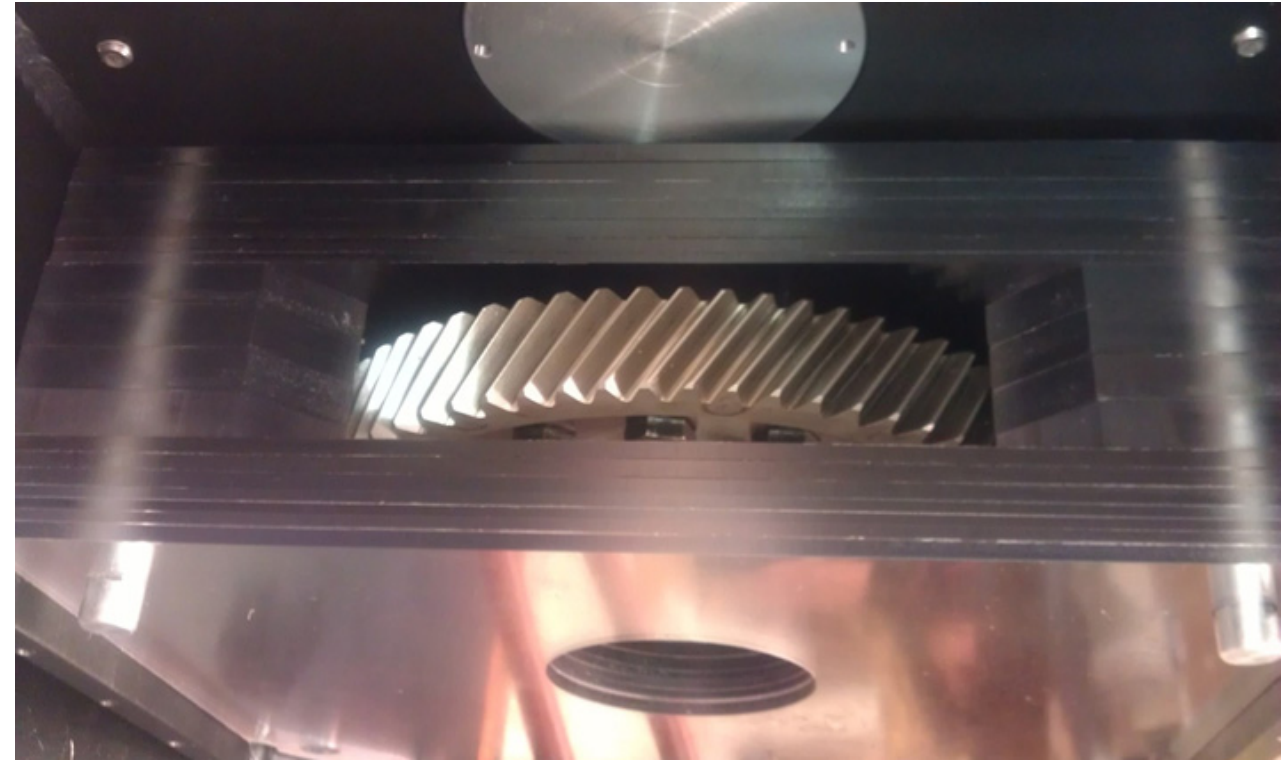

Figure 2.12 (a) Front and (b) top view of a set of enclosures assembled in the test gearbox. 
test bed. In order to accomplish this, several mechanical requirements had to be met. Specifically, the height of the input shaft (ring gear) center from the test bed surface must be the same height as the output coupling of the test machine. The end of the input shaft must be at the exact length of the matching spline of the output coupling. In addition to height requirement, precise mounting of the gearbox required a T-shaped support structure that held the gearbox rigidly on the test bed. Figure 2.13 shows a picture of the test gearbox mounted on the tests machine.

In this section, a brief explanation of the test machine and associated controls and instrumentation will be provided, while readers are referred to Ref. [6, 24, 25, 30, 34] for further details of the machine and repeatability of the measurements. Figure 2.14 shows a schematic of the top view lay-out of the test machine with the current gearbox mounted. The test machine comprised an AC motor driving a high-speed spindle connected by a 3:1 speed increasing belt drive. At the end of the spindle, a precision torque sensor is placed to measure the torque provided to the system being tested, in this case the churning gearbox. The drive system was powered by a 53 HP AC motor whose speed was digitally controlled to $\pm 2 \mathrm{rpm}$. The motor rotated the precision spindle that was integrated with the torque sensor at its other end. This torque sensor had a maximum speed capability of $25,000 \mathrm{rpm}$ and a maximum torque range of $50 \mathrm{Nm}$ with a resolution of $0.01 \%$ and an accuracy of $0.05 \%$ of full scale. The digital signal of the torque-meter was recorded and time-averaged to determine the torque required to drive the gearbox. Calibration of the torque sensor was carried out prior to, and periodically during, testing using the procedure developed by Chase [30]. 


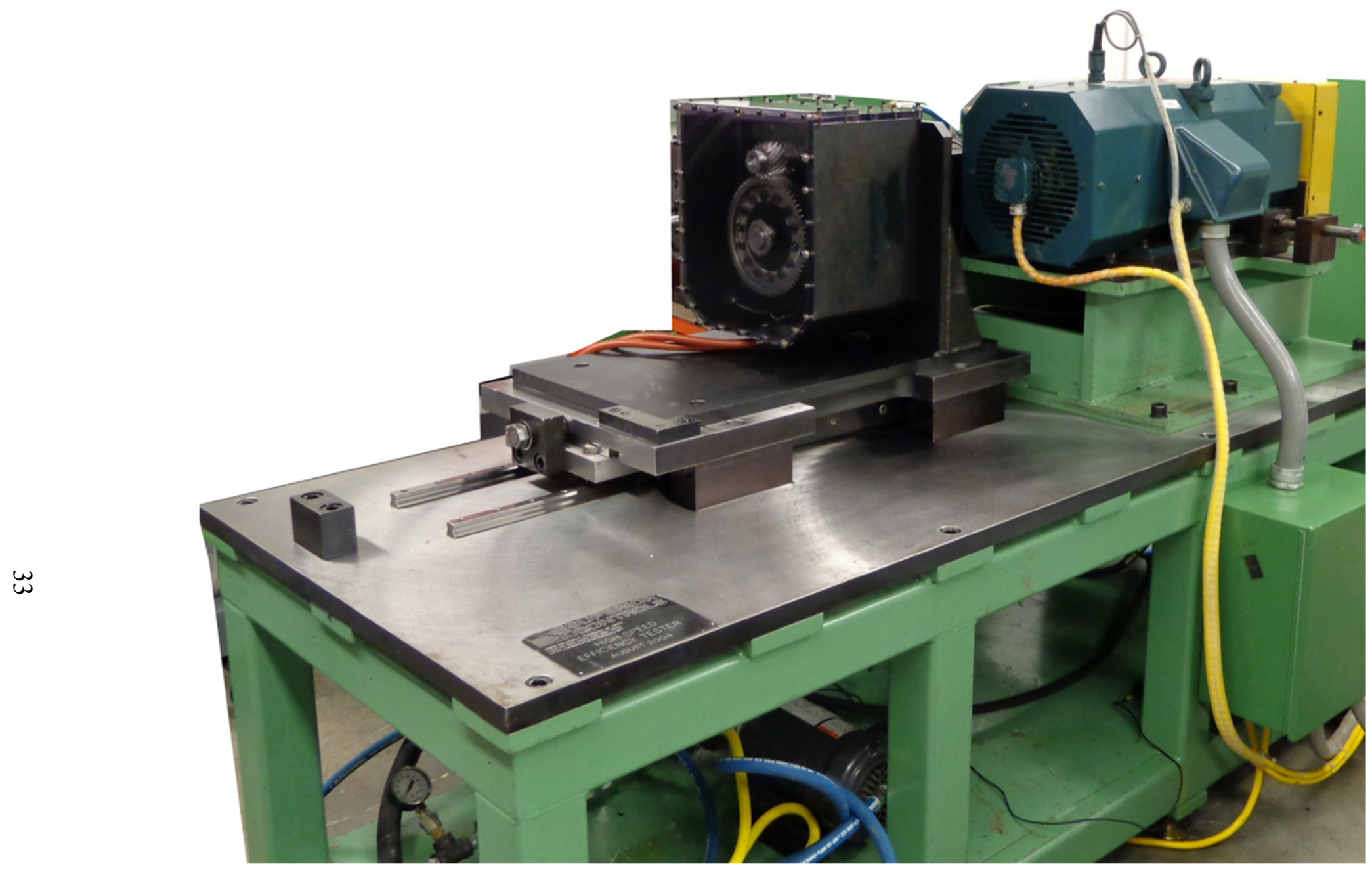

Figure 2.13 A picture of the high-speed efficiency test machine with the spin loss gearbox. 


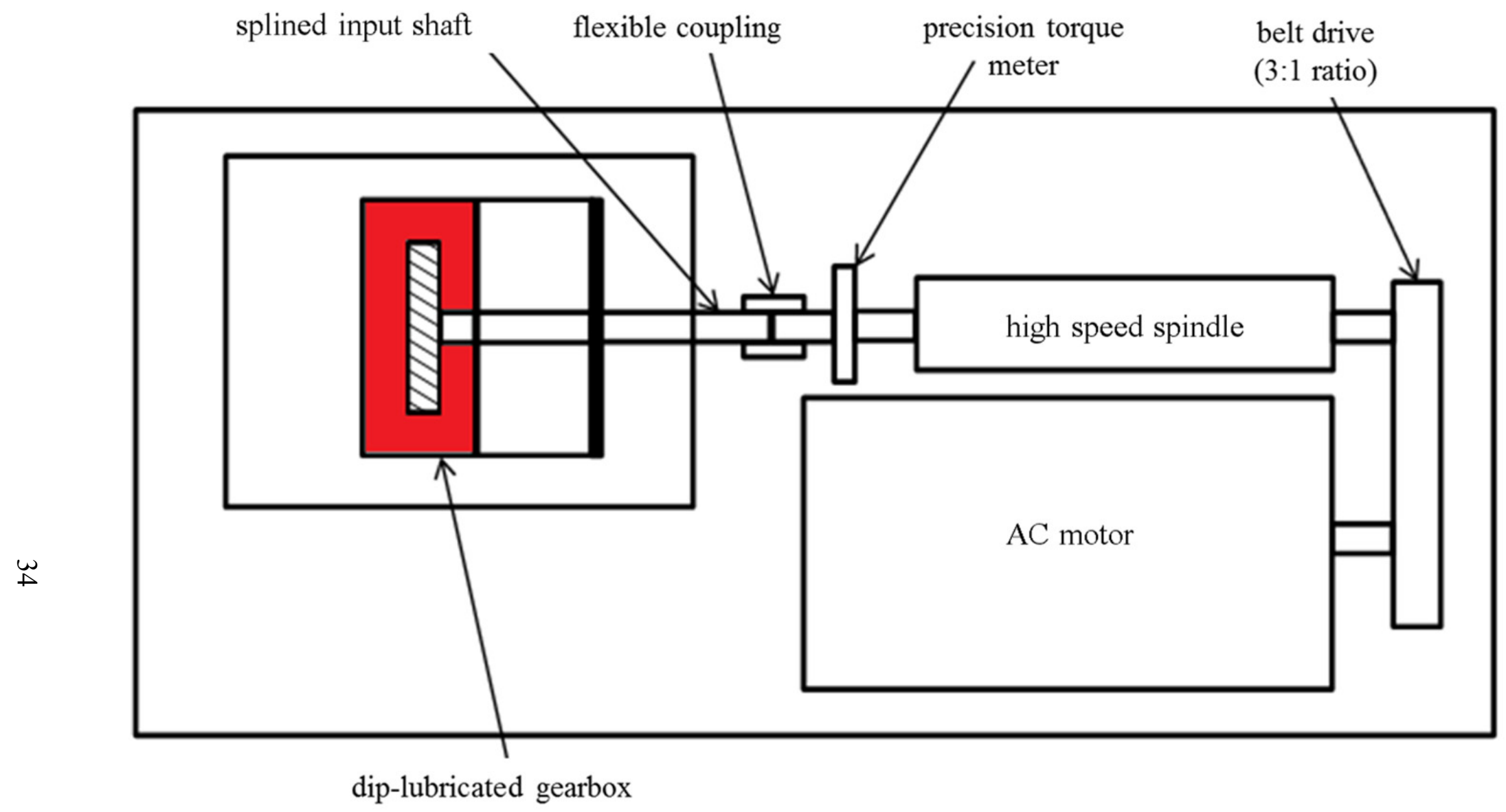

Figure 2.14 Top-view schematic of the test set-up with its key components. 
Since there is no output to the gearbox to apply a load, the torque measured by the torque sensor represents the torque loss, $T$. A flexible coupling provides means to engage the input shaft of the test gearbox to the torque sensor in a convenient way while protecting the sensor from adverse effects of minor shaft misalignments.

\subsection{Test Procedure}

Spin power losses, especially at low rotational speeds, can be of small magnitude. Thus, in order for spin power loss measurements to be accurate and repeatable a precise experimental procedure had to be developed and strictly followed. Set-up and maintenance of the test machine, gearbox, and measurement devices were vital to limit measurement errors. Before any test was run, the machine was operated for at least 30 minutes with heated lubricant in circulation in order to bring the entire gearbox up to the set steady-state test temperature. When the machine was sufficiently warmed up, the gearbox drive shaft was disconnected from the torque sensor. The torque sensor was then

zeroed. It was observed that the zero point did not drift much throughout the months of testing, indicating that torque readings remained consistent. In addition to resetting the torque sensor at the start of every testing period, the torque sensor was calibrated periodically using dead-weights and a torque arm. Through each calibration, it was noted that the previous calibration was maintained. This indicated that torque measurements were stable throughout this test program. After resetting the torque sensor, the drive spline was greased and re-installed so that testing could begin. 
One of the important test parameters, bulk lubricant temperature was the most difficult to maintain constant during testing. Instead of resorting to external heaters/coolers to regulate the lubricant temperature, an experience-based testing procedure was developed. Each tested lubricant, gear combination, lubricant level, and desired lubricant temperature produced some amount of heat that was dissipated in the gearbox. The steel gearbox conducted heat away from the high temperature lubricant. For some testing conditions heat was generated at a rate faster than the gearbox could conduct it away from the lubricant, while other conditions produced less heat than the gearbox was conducting away from the lubricant. These testing conditions would cause the bulk lubricant temperature to raise or drop, respectively. Therefore, tests were run in a sequence that constantly oscillated around the desired bulk lubricant temperature. This method of temperature control proved to be sufficient in this study, allowing the bulk lube temperature to be held to $\pm 5^{\circ} \mathrm{C}$ of the target temperature.

Measurement of the bulk lubricant temperature was done using an easy-to-attach low profile thermocouple bolted to a magnet at the bottom of the gearbox housing. This choice of thermocouple was made in an effort to minimize any disturbances introduced into the lubricant flow field. When testing using the polycarbonate enclosure pieces the thermocouple was bolted to the gear shaft bearing housing as it was the most exposed area left in the gearbox. 


\subsection{Test Matrix}

As stated in Chapter 1, effects of several different factors and parameters were aimed to be investigated in this study. This resulted in a rather large test matrix including multiple gear pairs or single gears/disks, multiple oil levels, multiple oil types and temperatures, various gear mesh positions and enclosure configurations. Only a sub-set of these measurements will be presented in the next chapter as many combinations produced trends similar to other combinations.

Table 2.2 summarizes the test matrix that was executed in this study. It consists of two families of tests, one with a single ring gear and the other with a gear pair. Spin loss of a gearbox, $P_{s}$, is given as the sum of gear drag losses, $P_{r d}$ and $P_{p d}$, gear mesh pocketing loss, $P_{p o c}$, bearing viscous losses, $P_{b}$, and seal losses, $P_{\text {seal }}$, as:

$$
P_{s}=P_{r d}+P_{p d}+P_{p o c}+P_{b}+P_{\text {seal }}
$$

When the system is operated at the single-shaft configuration of Figure $2.4(\mathrm{~b}), P_{p o c}=0$ and $P_{p d}=0$ since there is no pinion. With $P_{b}+P_{\text {seal }}$ separated through tests of input shaft at the same speed without the ring gear, drag power loss of the ring gear, $P_{r d}$, can be obtained from Eq. (2.1) through tests performed using the single gear configuration shown in Figure 2.4(b). As such, Table 2.2(a) specifies a number of single gear tests designed to describe the impact of various parameters on $P_{r d}$. Specific tests in this group are described as follows: 
Table 2.2 Test matrix considered in this study. (a) Single ring gear test matrix and (b) gear pair test matrix. Values with $(*)$ represent dimensions with no enclosure.

(a) Configuration: Single Ring Gear

\begin{tabular}{ccc}
\hline Parameter & Unit & Values \\
\hline Ring size (OD) & mm & $230.10,228.05,224.67$ \\
Ring type & -- & helical, spur, blank with no teeth \\
static oil level, $\bar{h}$ & -- & $0.05,0.25,0.5,00.75,1.00,1.50,2.00$ \\
Oil type & -- & $\mathrm{A}, \mathrm{B}, \mathrm{C}$ \\
Oil temperature & ${ }^{\circ} \mathrm{C}$ & $40,60,90$ \\
Axial clearance, $s$ & $\mathrm{~mm}$ & $1,5,12,50 *$ \\
Peripheral clearance, $p$ & $\mathrm{~mm}$ & $5,12, \geq 50 *$ \\
\hline
\end{tabular}

(b) Configuration: Gear Pair

\begin{tabular}{ccc}
\hline Parameter & Unit & Values \\
\hline Gear ratio, $n$ & -- & $4.56,4.25,3.94$ \\
Circumferential pinion angle, $\phi$ & degrees & $0,30,45,60,90,120,135,150,180$ \\
Static oil level, $\bar{h}$ & -- & $0.05,0.25,0.5,00.75,1.00,1.50,2.00$ \\
Oil type & -- & A, B, C \\
Oil temperature & ${ }^{\circ} \mathrm{C}$ & $40,60,90$ \\
\hline
\end{tabular}


(i) Influence of ring gear size. As shown in Figure 2.2(b) and specified in Tables 2.1 and 2.2, ring gears considered in this study have the same face width but slightly different outside diameters. All three ring gears were tested at different static oil levels, $\bar{h}$, to quantify the influence of outside diameter on $P_{r d}$.

(ii) Influence of teeth and helix angle. Ring gear of gear set \#3 in Table 2.1 and its replacements, an equivalent spur gear and a gear blank with no teeth (both at the same face width and outside diameter as the helical ring gear) as shown in Figure 2.6, were tested to investigate tooth effects on $P_{r d}$.

(iii) Effect of static oil level. The helical ring gear of gear pair \#3, as well as its spur and blank (no tooth) replacements have been tested at seven different static oil levels within $\bar{h} \in[0.05,2.00]$ shown in Figure $2.10(\mathrm{~b})$ to quantify the effect of $\bar{h}$ on $P_{r d}$

(iv) Influence of oil type and temperature. In order to investigate effects of oil viscosity and density, three different automotive oils, called lubricants A, B and C in Table 2.2 due to confidentiality reasons, were considered. These were tested at three temperature levels of 40,60 and $90^{\circ} \mathrm{C}$. Viscosity-temperature and densitytemperature curves for these three lubricants are provided in Figure 2.15.

(v) Enclosure effects. The ring gear of test gear pair \#3 was tested at $60^{\circ} \mathrm{C}$ with lubricant $\mathrm{B}$ in presence of various enclosure conditions. They included all combinations of axial clearance values $s=1,5$ and $12 \mathrm{~mm}$ and peripheral 


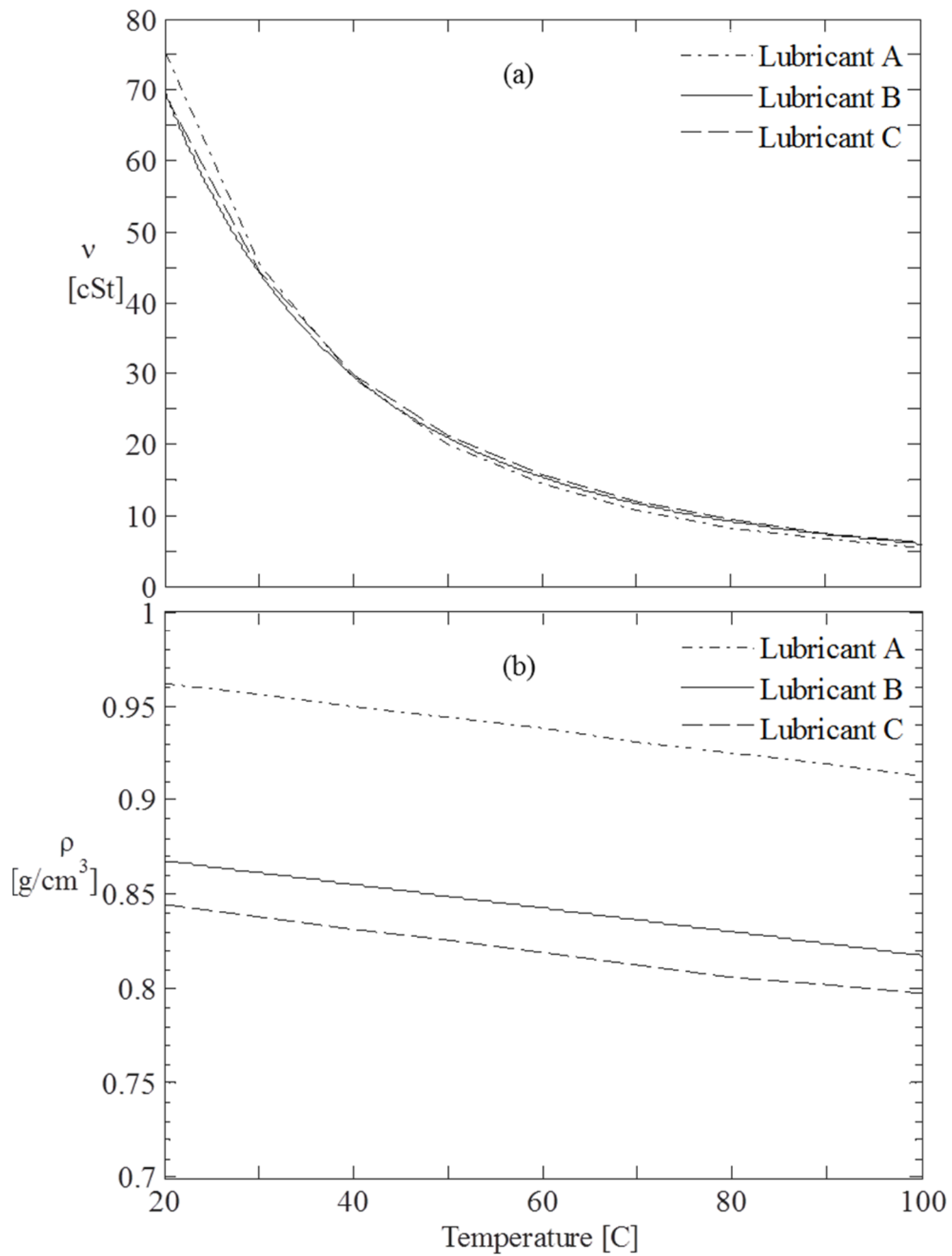

Figure 2.15 (a) Kinematic viscosity and (b) density as a function of temperature for the test lubricants considered in the study. 
clearance values of $p=5$ and $12 \mathrm{~mm}$. The $P_{r d}$ values for each $(s, p)$ test were compared to the no-enclosure case of $p \geq 50 \mathrm{~mm}$ and $s=50 \mathrm{~mm}$ to isolate enclosure effects.

The second group of tests in Table 2.2(b) are designed to investigate the total gear mesh power loss, $P_{S}$. When compared to the respective single ring gear tests, the pocketing component, $P_{p o c}$, will be isolated as a result of these tests according to Eq.

(2.1). The tests in this group include the following studies:

(i) Influence of gear ratio. All three gear ratios represented by each test gear pair were tested to study their influence on $P_{S}$.

(ii) Effect of static oil level. The test gear pair \#3 was tested at seven different static oil levels within $\bar{h} \in[0.05,2.00]$ shown in Figure 2.10 (b) to quantify the effect of $\bar{h}$ on $P_{S}$.

(iii) Effect of circumferential pinion position. According to Figure 2.10, nine different pinion positions will be tested within the range $\phi \in\left[0,180^{\circ}\right]$ under various $\bar{h}$ values to investigate the combined influence of $\phi$ and $\bar{h}$ on $P_{S}$. 


\section{CHAPTER 3}

\section{EXPERIMENTAL RESULTS}

\subsection{Introduction}

The test results hereinafter have been compiled from averaging individual test data gathered on the test machine described in Chapter 2. A sample of the raw test data is shown in Figure 3.1. Each test was nominally run for a minimum of 300 seconds. The first 100 seconds of data from each test was excluded from the averaging scheme to eliminate transients from previous test data. Here it is seen that the machine maintains the set input (ring gear) speed value, $\Omega_{r}$, at its set value very well, while the oil temperature levels (which is not controlled) remains relatively constant during a test. The measured torque loss, $T$, signal exhibits a certain amount of fluctuation. Hence, the torque loss, $T$, signal was averaged from $t=100 \mathrm{sec}$ to the end of the test to provide a representative value at this test condition. Each sub-study was run at an array of static 


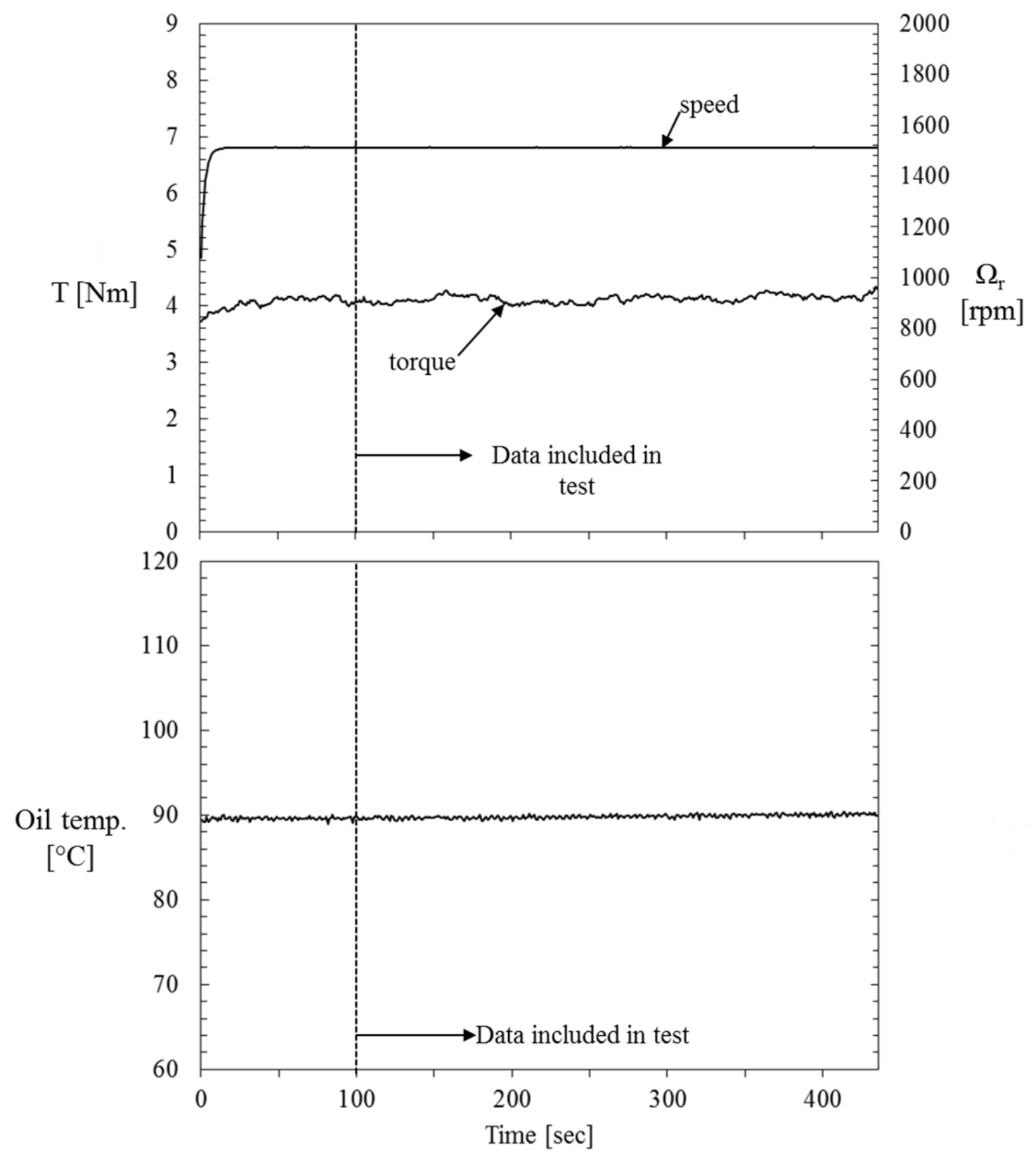

Figure 3.1 Sample segments of measured $\Omega_{r}, T$, and oil temperature time histories from a test at set values of $\Omega_{r}=1500 \mathrm{rpm}$ and $90^{\circ} \mathrm{C}$ with Lubricant B. 
lubricant levels, ring gear speeds, temperatures, and circumferential mesh locations (where applicable) as described in Table 2.2. Magnitudes of spin power loss, $P_{S}=\frac{2 \pi}{60} T \Omega_{r}\left(T\right.$ in N-m and $\Omega_{r}$ in rpm), for each plot have been normalized by a reference value, $P_{\text {ref }}$, for confidentiality purposes such that $\bar{P}_{s}=P_{s} / P_{r e f}$. It is noted here that the same $P_{r e f}$ value was used throughout this study such that each data set is comparable to others.

\subsubsection{Repeatability of Measurements}

In order to demonstrate the repeatability of the test machine and dip-lubricated gearbox, a ring gear test was repeated within $\bar{h} \in[0.05,2.0]$ and $\Omega_{r} \in[0,2000] \mathrm{rpm}$ at the end of the test program. This data was compared to an identical test run at the beginning of the test program. A sample of this comparison is shown in Figure 3.2 for $\bar{h}=1.0$ with lubricant B at $60^{\circ} \mathrm{C}$. The small deviation at $\Omega_{r}=2000 \mathrm{rpm}$ was likely due to the unsteady nature of the fluid flow at high speed. The data shows an average difference of only $3.4 \%$

over the entire speed range at $\bar{h}=1.0$, which was determined to be sufficient in terms of repeatability.

\subsubsection{Bearing and Seal Power Loss}

Bearing and seal components of power loss are a significant portion of overall spin power loss in a geared system. Therefore, in order to isolate the magnitude of power loss contribution from the gears, bearings and seal losses were measured first using the 


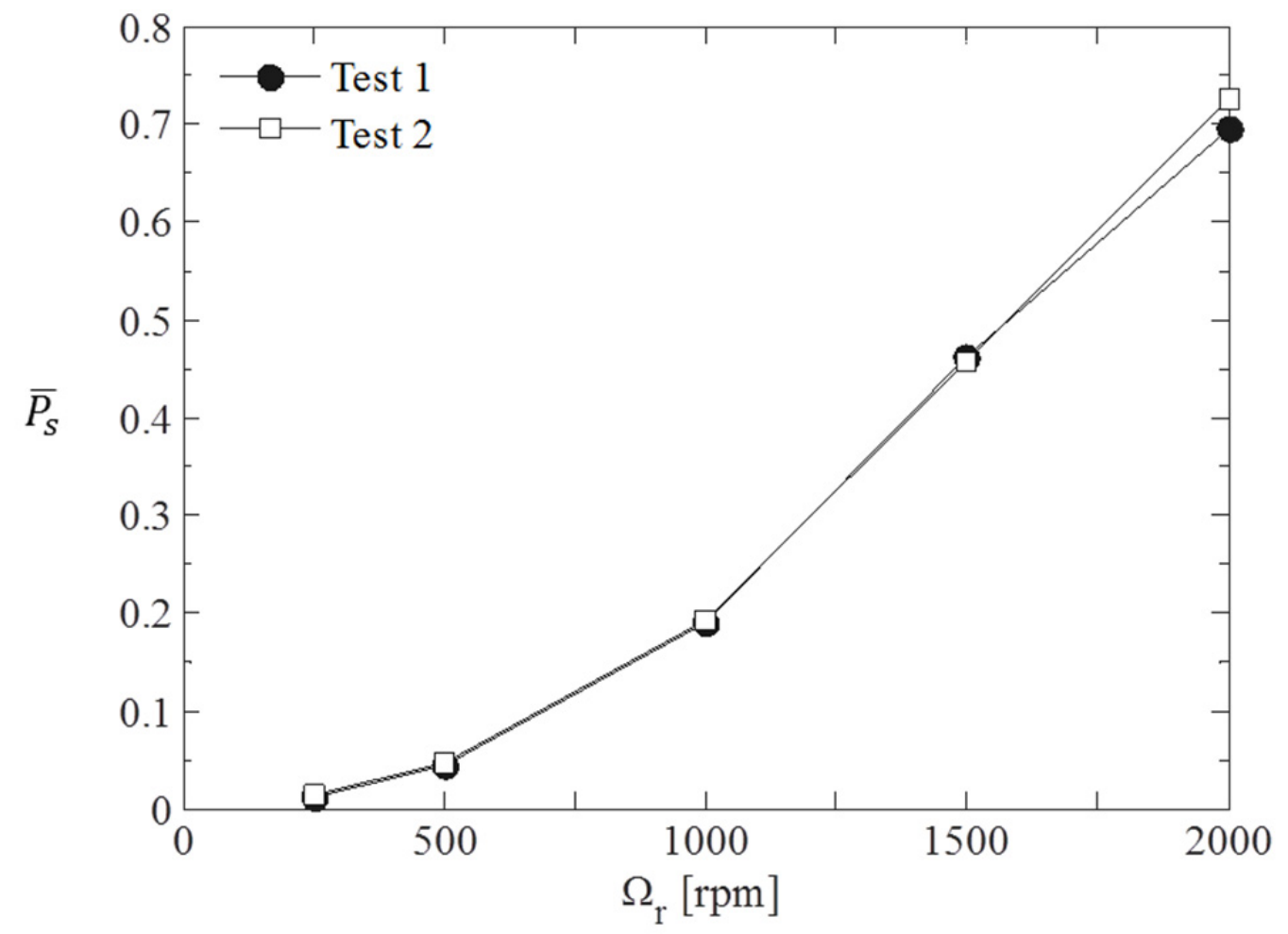

Figure 3.2 Repeatability of $\bar{P}_{s}$ measurements with ring gear \#3 operated in lubricant B at $60^{\circ} \mathrm{C}$ and $\bar{h}=1.0$. 
configuration of Figure 2.4(b) with no ring gear such that $P_{r d}+P_{p d}+P_{p o c}=0$ in Eq. (2.1).

The design of the gearbox exposed the outboard bearings (those closest to the gear/pinion) to the test lubricant. Therefore, two tests were run using isolated bearings with two different oil levels in the bearings. The first test, referred to as the $d r y$ bearing test, represented experiments where $\bar{h} \in[0.05,0.75]$. This test exposed the outboard bearing to a very small amount of oil to simulate an oil mist environment within the bearing. The second test, referred to as the wet bearing test was intended to represent experiments where $\bar{h} \in[1.0,2.0]$. This test partially submerged the outboard bearing to simulate an oil bath environment. The inboard bearings were half immersed in oil and sealed off from the test gearbox chamber as shown in Figure 2.4(b).

Figure 3.3 shows the variation of bearing power loss as a function of ring gear shaft speed, $\Omega_{\text {in }}=\Omega_{r}$, under both dry and wet conditions using lubricant B at a bulk lubricant temperature of $60^{\circ} \mathrm{C}$. It is seen in Figure 3.3 that dry conditions result in 30 to $50 \%$ less bearing power loss than wet conditions. Both tests follow an exponential trend with increasing $\Omega_{r}$, allowing simple regression of each set of data to be used to remove bearing power losses from the gear tests.

Also shown in Figure 3.3 are the power series curve fits of the experimental data compared to viscous power loss empirical relations defined by Harris [35]. Harris' 


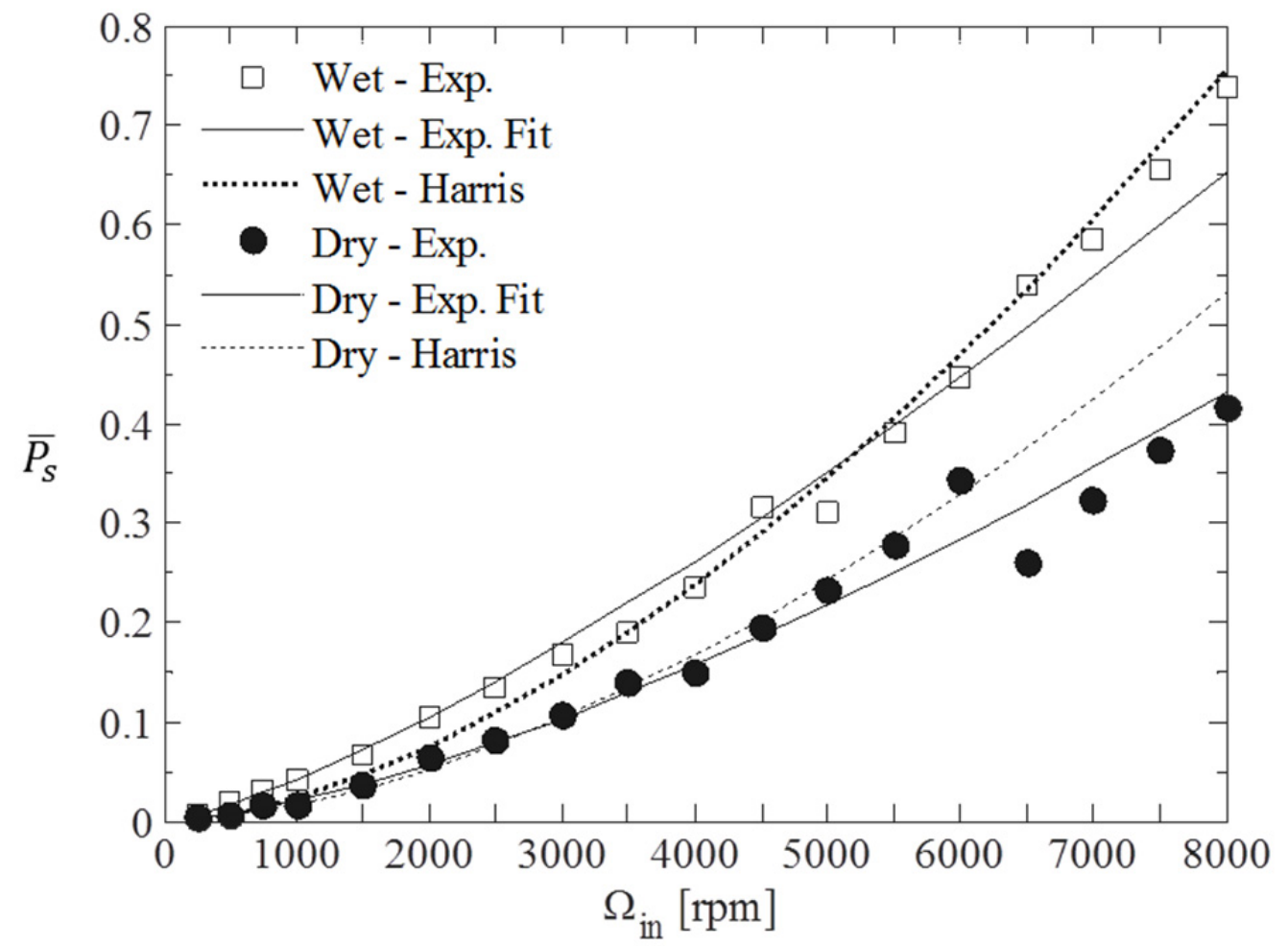

Figure 3.3 Measured input shaft bearing and seal power loss from wet and dry bearing loss tests with lubricant $\mathrm{B}$ at $60^{\circ} \mathrm{C}$. Also shown is the comparison formulae of Harris and Kotzalas [35]. 
empirical formula has the form $\bar{P}_{S}=a \Omega_{r}^{b}$. For comparison, the coefficients $(a, b)$ for the dry test are given as $\left(8.44(10)^{-4}, 1.463\right)$, whereas the Harris' formulae yield $\left(1.66(10)^{-4}, 1.67\right)$. The wet tests result in $(a, b)=\left(4.56(10)^{-3}, 1.32\right)$, whereas the Harris'

formulae yield $(a, b)=\left(0.24(10)^{-3}, 1.67\right)$. Harris' formulae are seen to agree reasonably well to the experimental data presented. However, at higher speeds (such as those experienced by the pinion bearings) Harris' formulae tend to over predict the bearing viscous losses seen in this experiment.

\subsubsection{Vortex Development}

The oil churning experiments performed in this study were subject to different fluid regimes based on $\Omega_{r}$ and $\bar{h}$ values used. When $\bar{h}<1.5$ and ring gear speeds $\Omega_{r}<1500 \mathrm{rpm}$, flow of fluid was such that an operating oil level could be defined at lower elevation than the static oil level, where oil thrown upward in in the direction of tangential velocity of the gear would return back to the oil bath at the bottom. An example of this flow regime is shown Figure 3.4(a). Meanwhile, for $\bar{h} \geq 1.5$ and $\Omega_{r} \geq 1500 \mathrm{rpm}$, forces imposed on the fluid were large enough for the fluid to form a complete and continuous circulation path within the gearbox in the direction of $\Omega_{r}$, which here will be loosely referred to as a vortex. Such a condition is shown in Figure 3.4(b). $\quad \bar{P}_{S}$ values for test conditions resulting in a vortex were often 2 to 4 times higher 
(a)

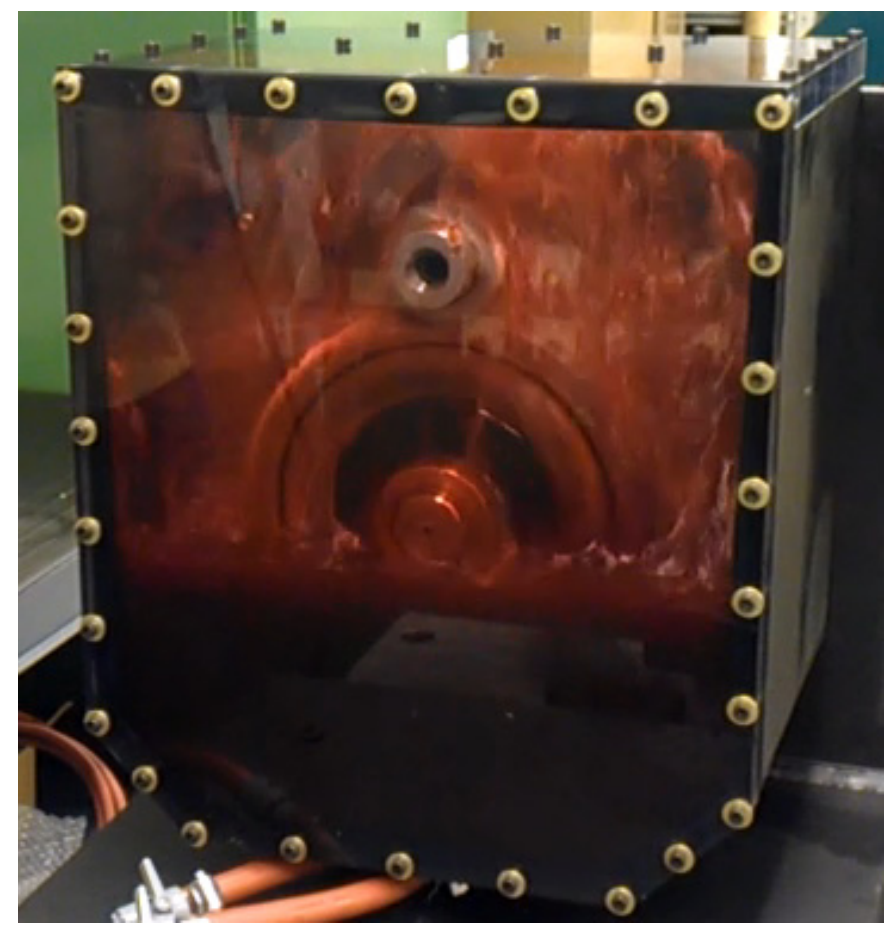

(b)

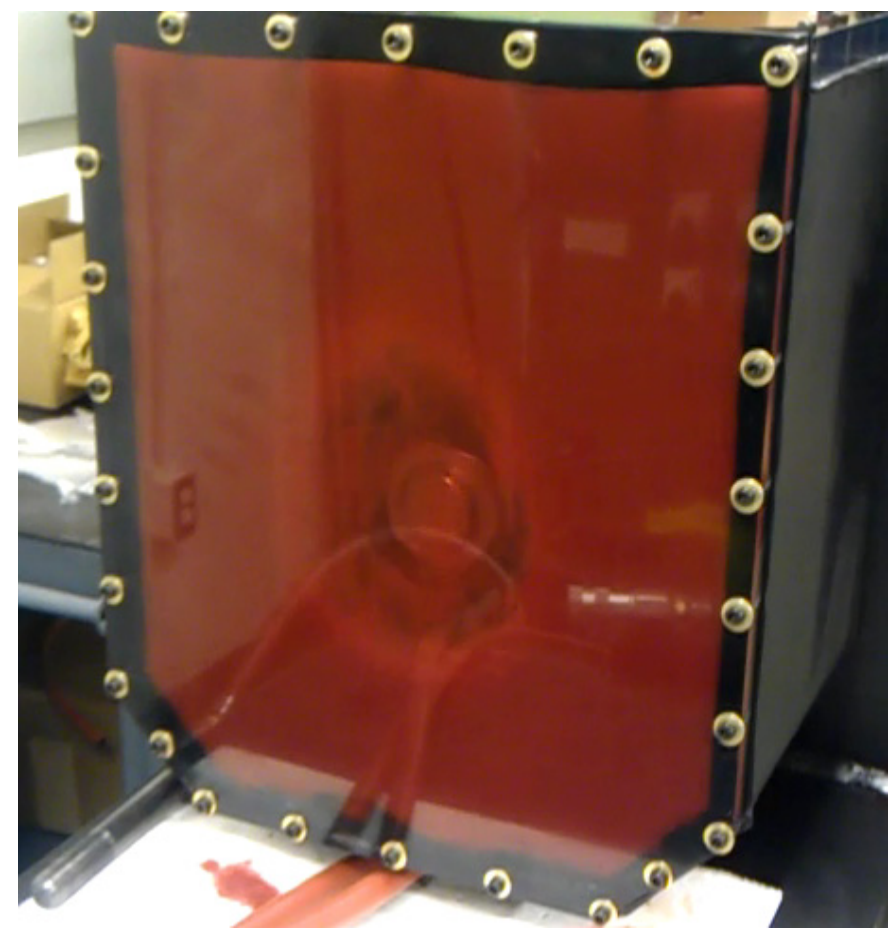

Figure 3.4 Examples of oil flow (a) without a vortex and (b) with a vortex. 
than that of tests without a vortex. This resulted in sudden changes in the trend lines, dividing the data into two $\Omega_{r}$ ranges, a low-speed range with flow conditions such as that shown in Figure 3.4(a) and a high-speed range with a vortex as the seen in Figure 3.4(b). At the conditions when a vortex is present, the bulk lubricant temperature within the gearbox experienced a rise of about $2^{\circ} \mathrm{C} / \mathrm{min}$ when operating with a single gear and $12^{\circ} \mathrm{C} / \mathrm{min}$ when operating with a gear pair.

While valid conclusions can still be made when a vortex develops, they should be made with caution. Vortex development is gearbox specific and depends on the geometry of the housing (among other factors). Direct comparisons between tests performed here are possible. However, data obtained under a vortex condition might not be suitable for comparisons to other gearboxes having different shapes and dimensions.

\subsection{Drag Losses of a Single Ring Gear}

This section presents results of experiments performed using a single ring gear in the configuration shown in Figure 2.4(b). As defined in Table 2.2(a), the single gear experiments included a number of variations ranging from gear size and tooth shape to the lubrication conditions. The power loss results presented in this section consist of ring gear drag and input shaft bearing/seal losses, i.e. $P_{s}=P_{r d}+P_{b}+P_{\text {seal }}$. However, differences between respective tests correspond directly to differences in $P_{r d}$ since $P_{b}+P_{\text {seal }}$ components should be the same. 


\subsubsection{Effect of Ring Gear Size}

Figure 3.5 compares ring gear spin losses for the three ring gears shown in Figure 2.2(b) with outside diameters defined in Table 2.1. These tests were performed with lubricant B at $60^{\circ} \mathrm{C}$. Here the differences in $\bar{P}_{S}$ are solely due to the slight differences in the outside diameter. For all four static oil levels presented in Figure $3.5(\bar{h}=0.05,1.0$, 1.5 and 2.0), losses for the largest diameter gear (ring gear \#1) are observed to be highest, followed by ring gears $\# 2$ and $\# 3$ in the order of their outside diameter. Since the sizes of ring gears are rather close (outside diameters of 230.10, 228.05 and $224.67 \mathrm{~mm}$ ), the differences in $\bar{P}_{S}$ are small. For instance, in Figure 3.5(b), the difference of $\bar{P}_{S}$ for ring gears $\# 1$ and $\# 3$ is only $11 \%$ at $\Omega_{r}=2000 \mathrm{rpm}$ and $\bar{h}=1.0$. Similarly, in Figure 3.5(d) for $\bar{h}=2.0$, a maximum difference of $15.5 \%$ between ring gear $\# 1$ and $\# 3 \bar{P}_{S}$ is observed for $\Omega_{r} \leq 1000 \mathrm{rpm}$ where the oil flow is similar to that seen in Figure 3.4(a). $\bar{P}_{S}$ values do not correlate to the ring gear size for $\Omega_{r}>1000 \mathrm{rpm}$ primarily due to vortex formation. Also observed in Figure 3.5 is an exponential increase of $\bar{P}_{S}$ with $\Omega_{r}$ as well as significant increases in $\bar{P}_{S}$ with $\bar{h}$, both of which will be quantified later.

\subsubsection{Effect of Lubricant Type and Static Oil Level}

Figure 3.6 shows the combined effects of lubricant type and submersion level on

$\bar{P}_{s}$. The measured $\bar{P}_{s}$ values for all three lubricants are compared to each other within $\bar{h} \in[0.05,2.0]$ at discrete speed values of $\Omega_{r}=250,500,750,1000,1500$ and $2000 \mathrm{rpm}$. 

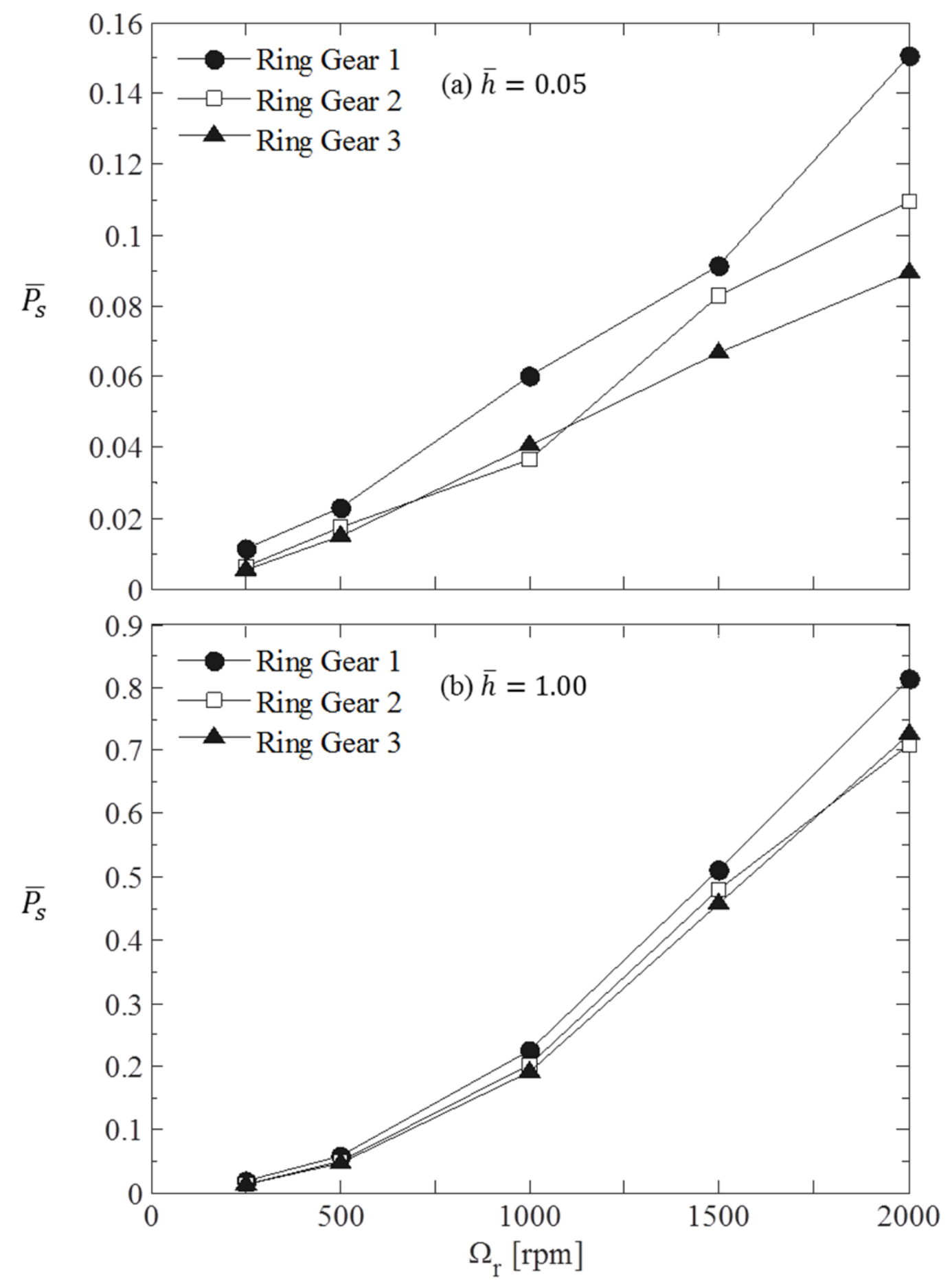

Figure 3.5 Influence of ring gear diameter on $\bar{P}_{S}$ at (a) $\bar{h}=0.05$, (b) $\bar{h}=1.0$, (c) $\bar{h}=1.5$, and (d) $\bar{h}=2.0$. Lubricant B at $60^{\circ} \mathrm{C}$. (continued) 
Figure 3.5 (continued)
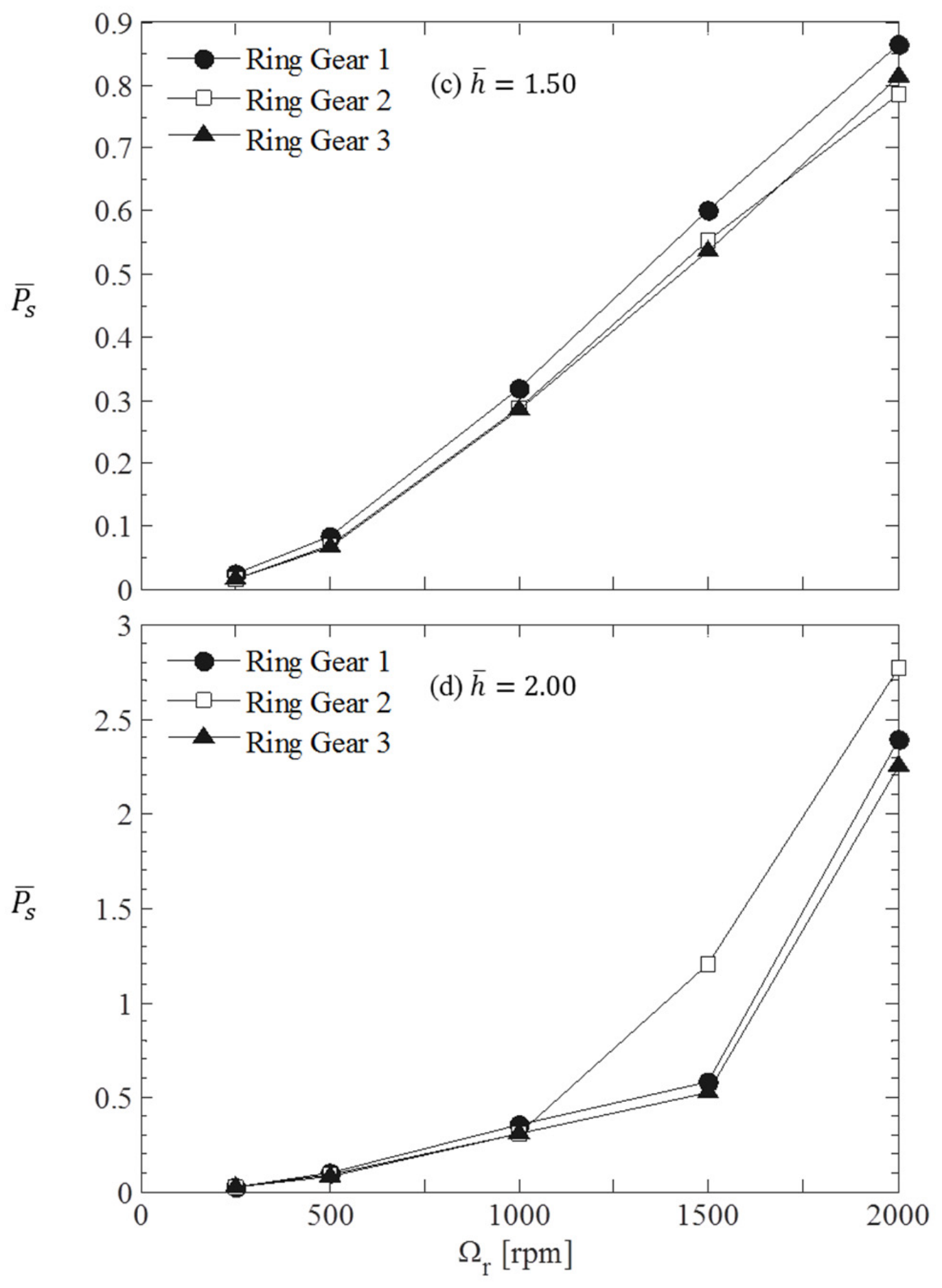

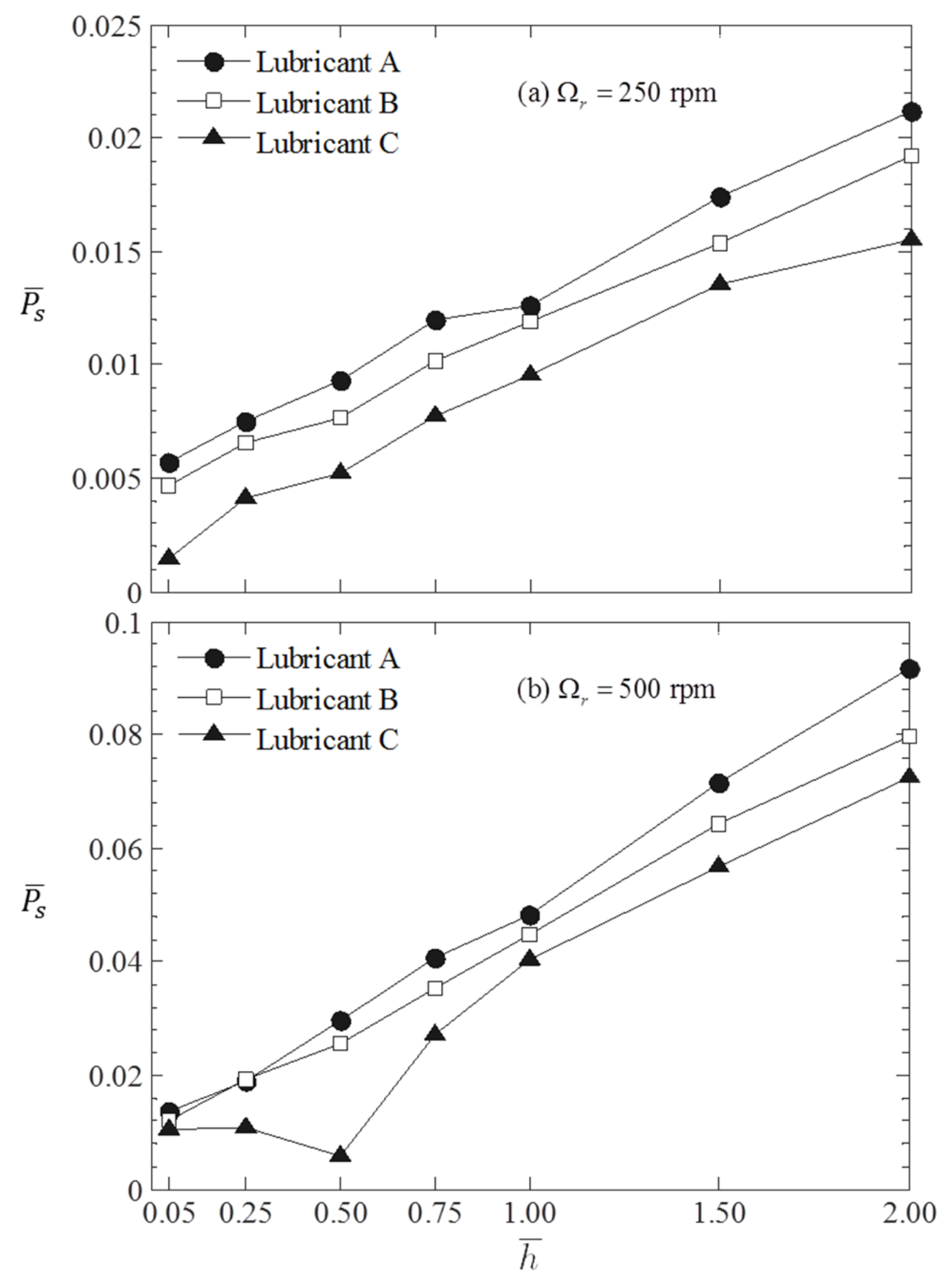

Figure 3.6 Combined influences of lubricant type and $\bar{h}$ on $\bar{P}_{S}$ at $60^{\circ} \mathrm{C}$ and (a)

$$
\begin{aligned}
& \Omega_{r}=250 \mathrm{rpm}, \text { (b) } \Omega_{r}=500 \mathrm{rpm},(\mathrm{c}) \Omega_{r}=750 \mathrm{rpm},(\mathrm{d}) \Omega_{r}=1000 \mathrm{rpm},(\mathrm{e}) \\
& \Omega_{r}=1500 \mathrm{rpm} \text {, and (f) } \Omega_{r}=2000 \mathrm{rpm} \text {. (continued) }
\end{aligned}
$$


Figure 3.6 (continued)
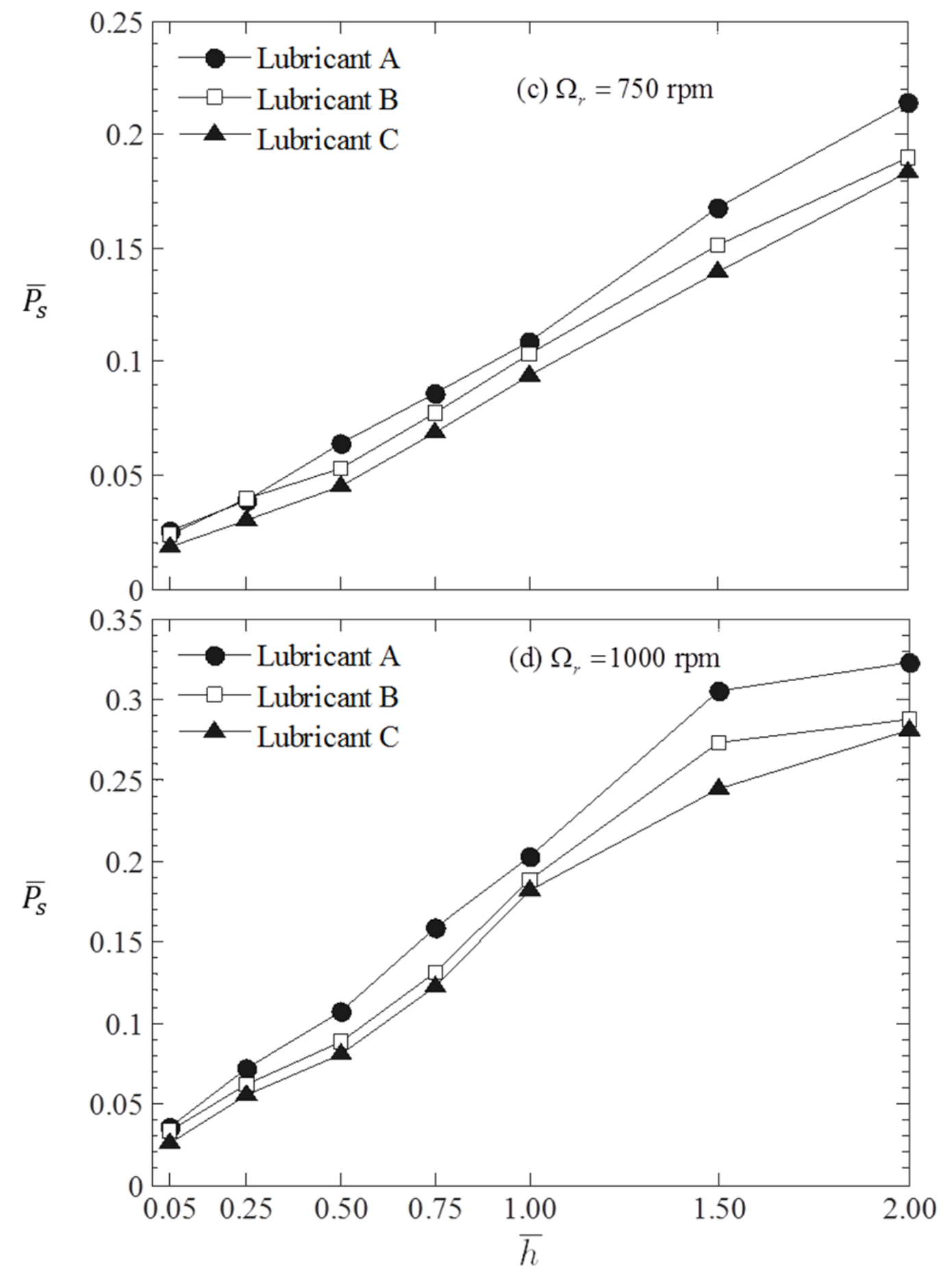

(continued) 
Figure 3.6 (continued)
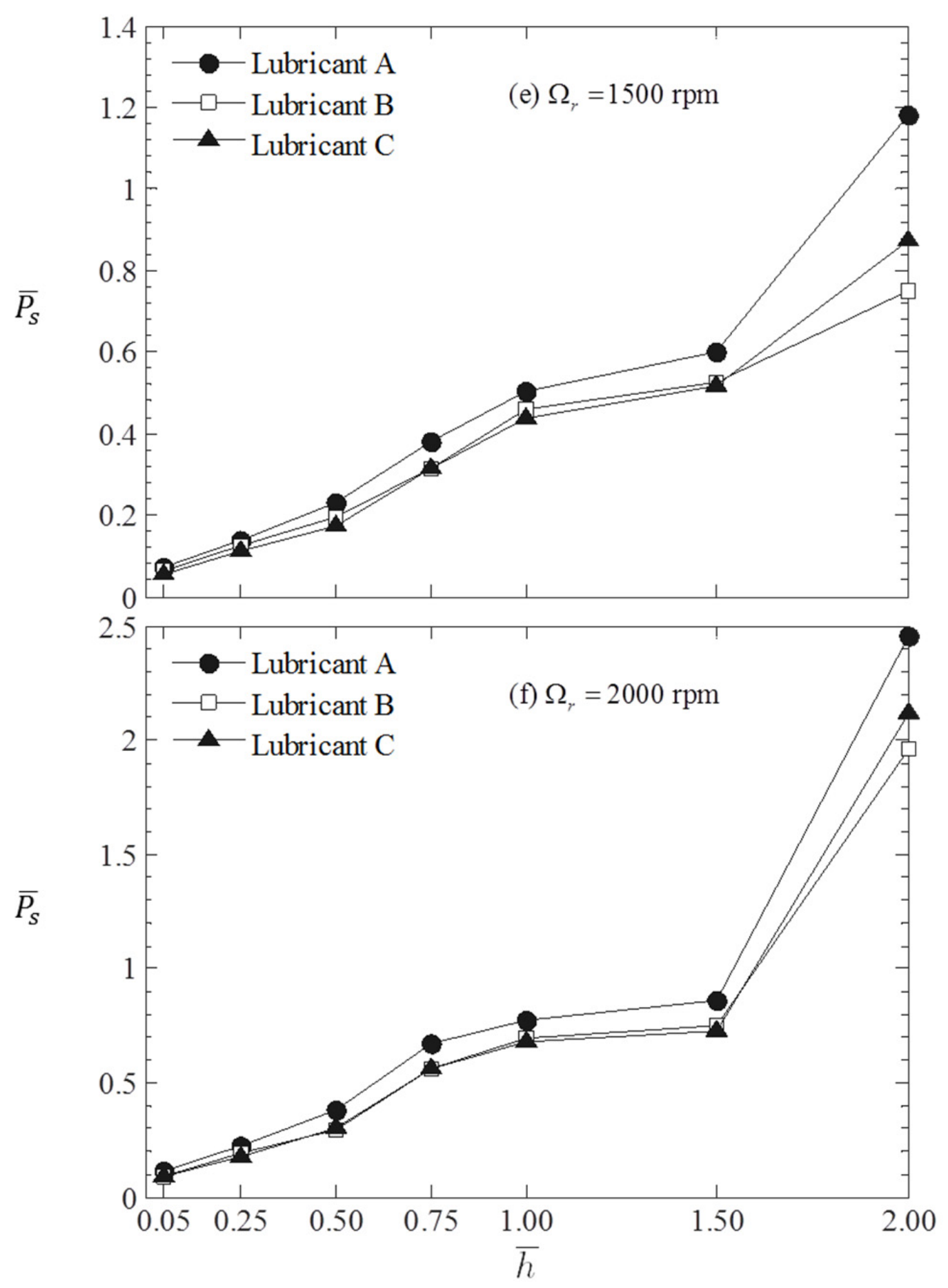
The differences between the viscosity of each lubricant at any given temperature is negligible, as seen in Figure 2.15(a), however a significant difference in density exists between the lubricants. The lubricant density values are shown in Figure 2.15(b) for lubricants $\mathrm{A}, \mathrm{B}, \mathrm{C}$ at $60^{\circ} \mathrm{C}$ and are $0.938,0.843$, and $0.819 \mathrm{~g} / \mathrm{cm}^{3}$, respectively. The lubricant type (i.e. lubricant density) has a profound impact on churning losses, as seen by an average difference of $19 \%$ between lubricants A and C at full submersion levels ( $\bar{h}=2.0)$ operating in non-vortex conditions, while an average difference of only $7 \%$ exists between lubricants B and C under the same conditions. Vortex formations at the same speeds and immersion levels indicate that lubricant type does not influence vortex development within the resolution of $\Omega_{r}$ used in this study.

The influence of $\bar{h}$ on $\bar{P}_{s}$ is also evident in Figure 3.6 regardless of $\Omega_{r}$ and oil type. An almost linear relationship is measured between $\bar{h}$ on $\bar{P}_{S}$ at all speed values considered for regions of non-vortex flow. The slopes of these curves are rather steep pointing to significant changes to $\bar{P}_{S}$ with $\bar{h}$. For instance, Figure 3.6(c) at $\Omega_{r}=750$ rpm shows $\bar{P}_{S}=0.02,0.11$ and 0.21 at $\bar{h}=0.05,1.0$ and 2.0, respectively. This indicates that a fully submerged gear has an order of magnitude higher $\bar{P}_{S}$ than a gear that is barely touching the static oil surface. These differences are attributable to ring gear drag losses. Also observed in Figures 3.6(e,f) is the trend between $\bar{P}_{S}$ and $\bar{h}$ is not linear for $\Omega_{r} \geq 1500 \mathrm{rpm}$ where oil flow starts to form a vortex especially at higher $\bar{h}$ values. 


\subsubsection{Effect of Lubricant Temperature}

Figure 3.7 illustrates the effect of bulk lubricant temperatures on $\bar{P}_{S}$ using test results at 40,60 and $90^{\circ} \mathrm{C}$ with lubricant $\mathrm{B}$. The kinematic viscosity values for this lubricant at these three temperature values are 29.8, 15.4, 7.3 cSt, respectively. Figure 3.7 indicates that at low immersion levels (say $\bar{h} \leq 1.0$ ), the influence of bulk lubricant temperature on $\bar{P}_{S}$ is negligible within the $\Omega_{r}$ range considered. Oil temperature (i.e. kinematic viscosity) becomes more influential for $\bar{h}=1.5$ and 2.0. Figures 3.7(c) and (d) show an average $23 \%$ increase in $\bar{P}_{S}$ when decreasing oil temperature from $90^{\circ} \mathrm{C}$ to $40^{\circ} \mathrm{C}$ in an environment without a vortex.

Also noteworthy is that a vortex forms in Figure 3.7(d) at $\Omega_{r}=1500 \mathrm{rpm}$ and $90^{\circ} \mathrm{C}$, whereas no vortex is evident at $40^{\circ} \mathrm{C}$ or $60^{\circ} \mathrm{C}$ tests at the same speed. Similarly, at $\Omega_{r}=2000 \mathrm{rpm}$, a vortex develops at 60 and $90^{\circ} \mathrm{C}$, but not at $40^{\circ} \mathrm{C}$, suggesting that the change in this flow regime perhaps has to do with transition from laminar to turbulent regime with the change in Reynolds number, as suggested by Seetharaman and Kahraman [33]. These results indicate that temperature (i.e. viscosity) has a strong influence on vortex development. 

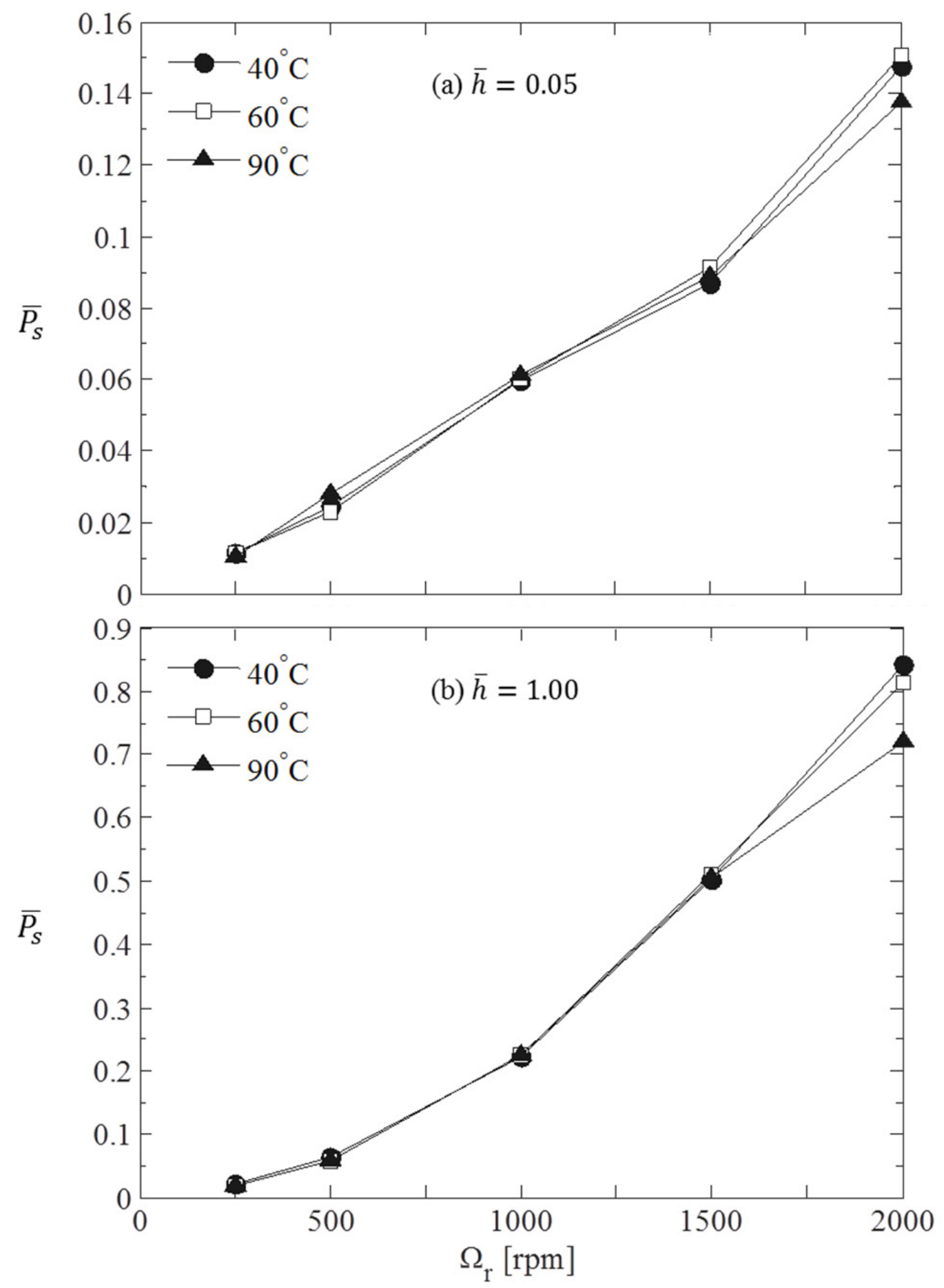

Figure 3.7 Effect of bulk lubricant temperature on $\bar{P}_{S}$ with Lubricant B at (a) $\bar{h}=0.05$, (b) $\bar{h}=1.0$, (c) $\bar{h}=1.5$, and (d) $\bar{h}=2.0$. (continued) 
Figure 3.7 (continued)
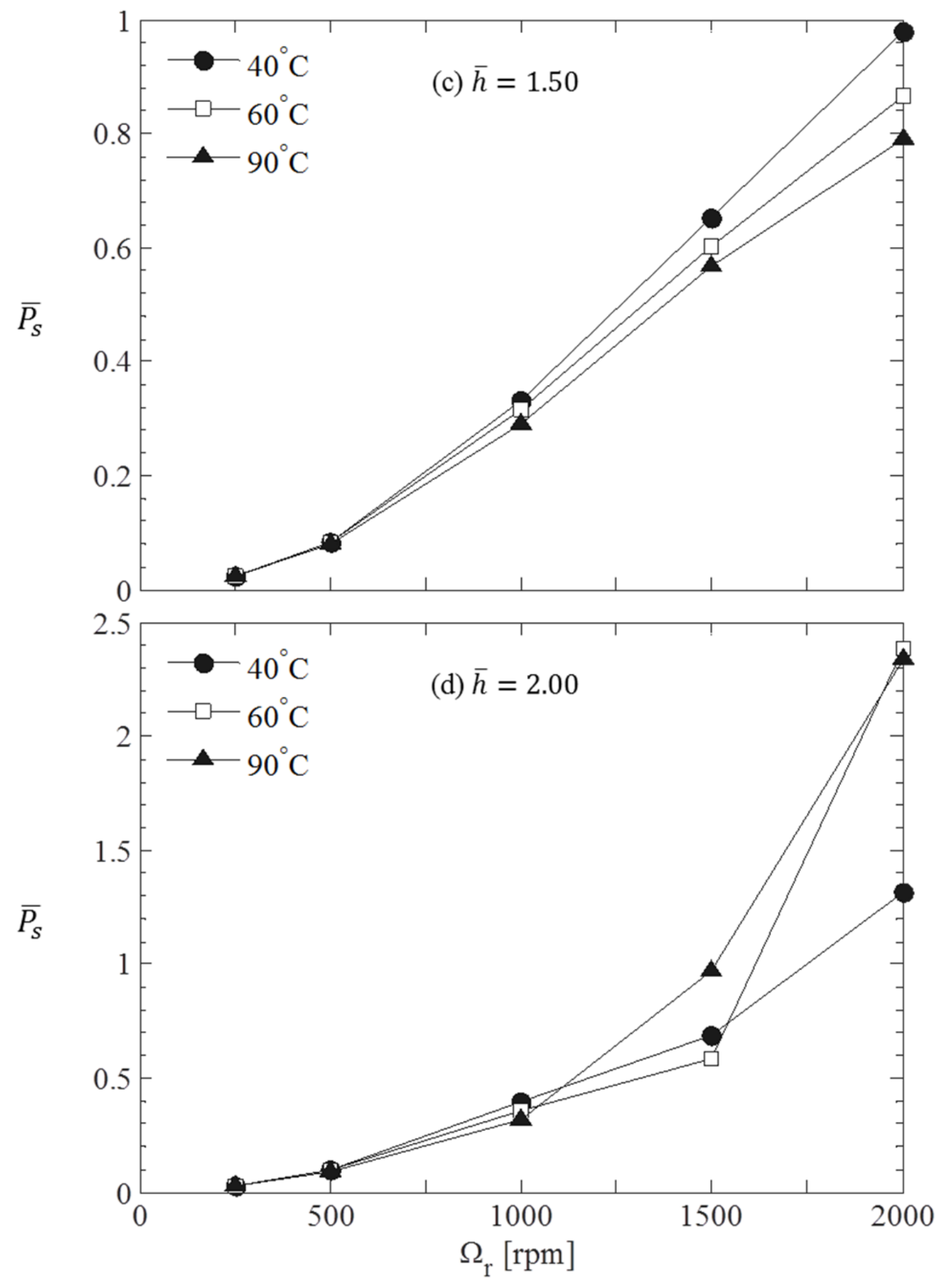


\subsubsection{Influence of Gear Teeth and Helix Angle}

One of the main simplifying assumptions in modeling of drag losses has been that gear teeth have no influence. With this, various recent models (e.g. [17-19, 33]) approximated the gear to be a cylindrical disk with a diameter equal to the outside diameter of the gear being modeled. There is very little experimental data to verify or discard this assumption. Likewise, there is no data on the influence of helix angle (spur versus helical gear). As such, the single gear test matrix of Table 2.1 included a study to specifically investigate this issue using a helical ring gear, a spur gear equivalent and a cylindrical disk replacement of the gears as shown in Figure 2.6(d,e).

Figure 3.8 shows the influence of gear teeth on $\bar{P}_{s}$. Figure 3.8(a) for $\bar{h}=0.05$ indicates that gears (spur or helical) cause consistently more $\bar{P}_{S}$ than a disk with no teeth (called "Gear Blank" in the figure). At $\Omega_{r}=2000 \mathrm{rpm}$ and $\bar{h}=0.05$, the helical gear and spur gear exhibit $84 \%$ and $76 \%$ more drag power loss than the gear blank, respectively.

As $\bar{h}$ increases, differences between spur and helical gears become more

pronounced. For instance, Figure 3.8(d) for $\bar{h}=2.0$ shows a 34\% difference between the spur gear and blank, while only a 9\% difference between the spur and helical gear at $\Omega_{r}=1000 \mathrm{rpm}$. The spur gear was shown to have a consistently larger $\bar{P}_{S}$ than the helical gear for cases when no vortex is formed. The helical gear tests exhibited vortex development at $\Omega_{r} \geq 1500 \mathrm{rpm}$ while spur gear was shown to develop a vortex only at 


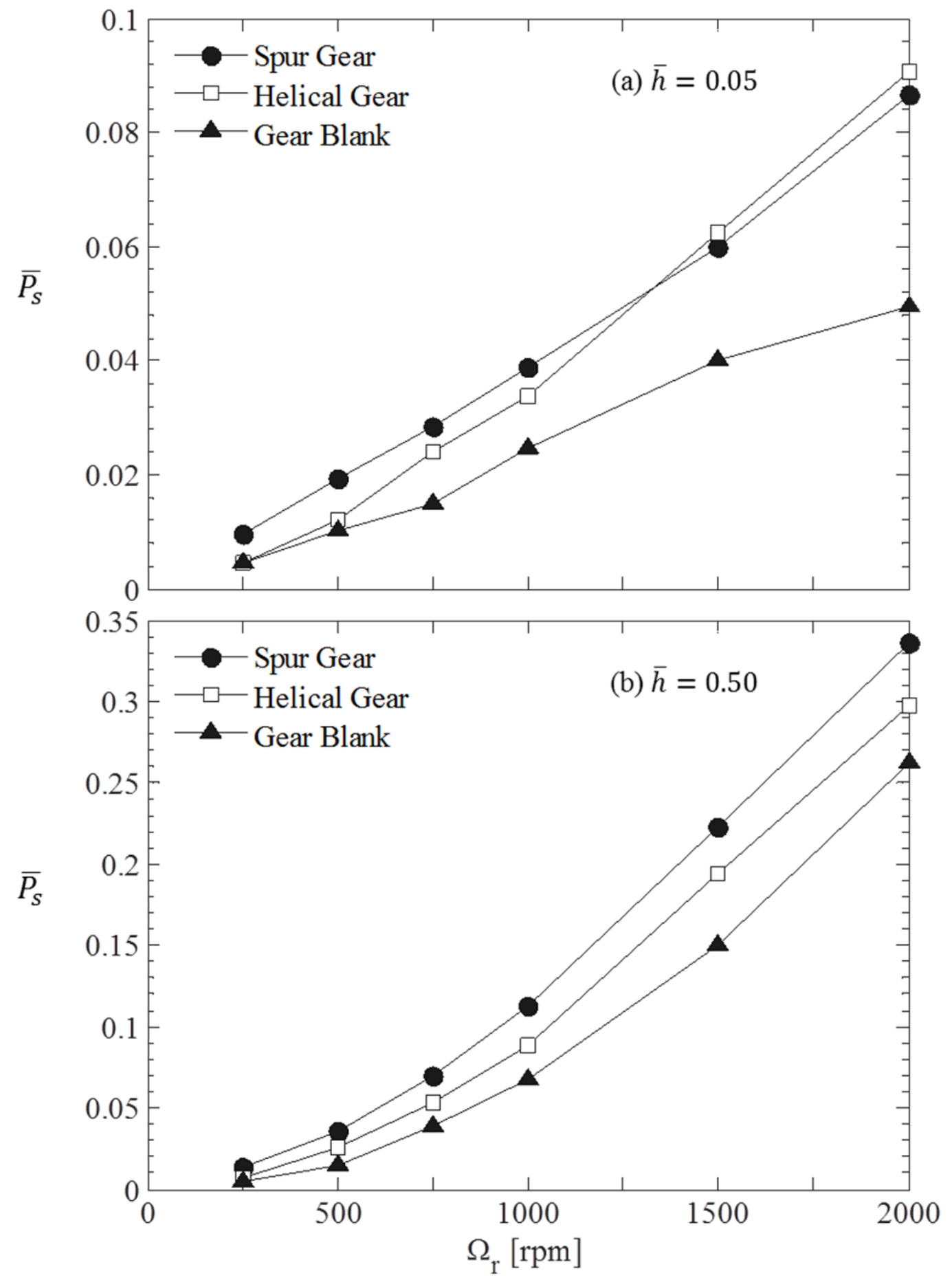

Figure 3.8 Effect of gear teeth on $\bar{P}_{s}$ within a range of $\Omega_{r}$ at (a) $\bar{h}=0.05$, (b) $\bar{h}=0.5$, (c) $\bar{h}=1.0$, and (d) $\bar{h}=2.0$. Lubricant B at $60^{\circ} \mathrm{C}$. (continued) 
Figure 3.8 (continued)
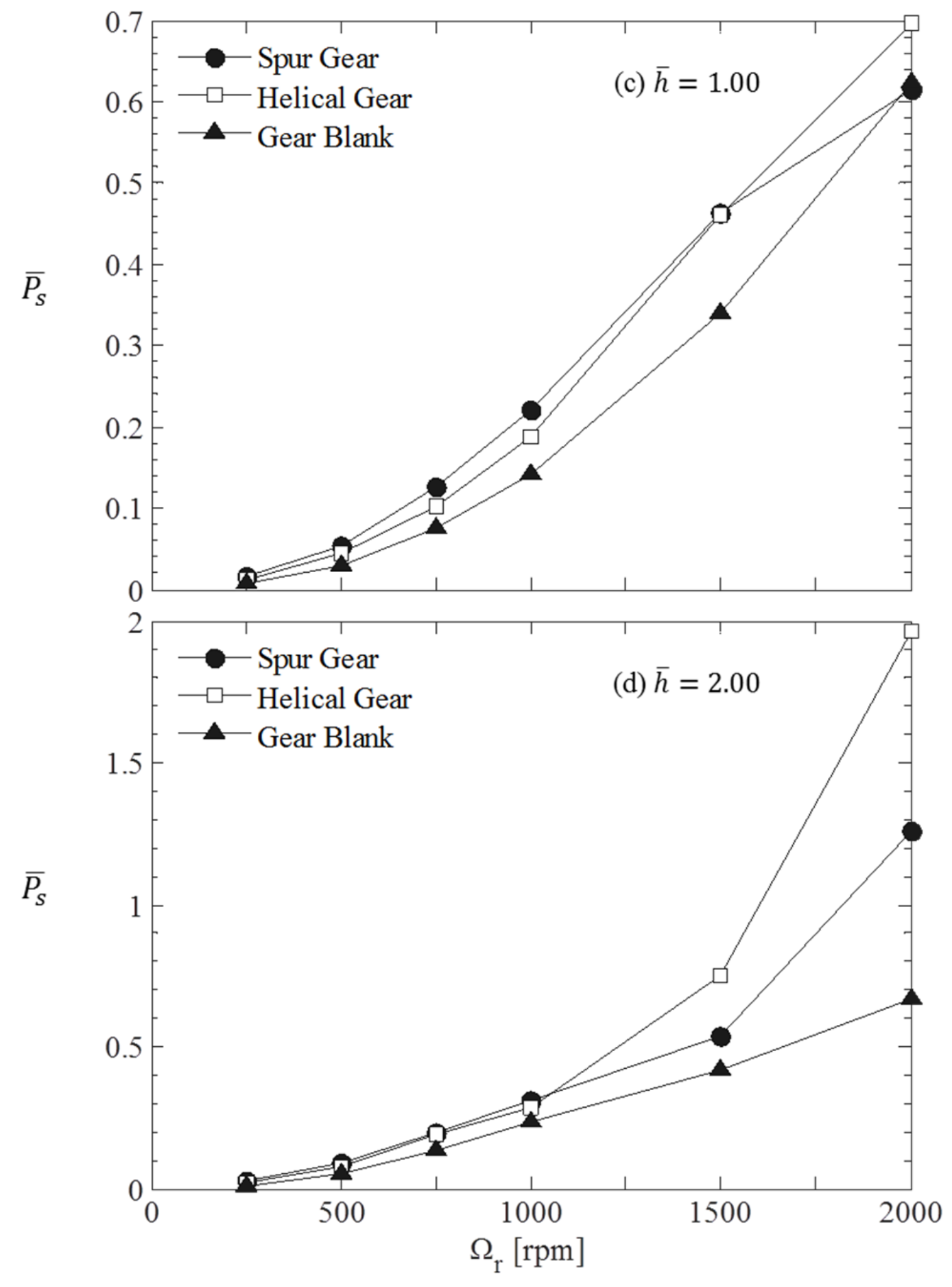
$\Omega_{r}=2000 \mathrm{rpm}$. As a result of this, the helical gear in Figure $3.8(\mathrm{~d})$ for $\bar{h}=2.0$ had higher $\bar{P}_{S}$ values than the spur gear for $\Omega_{r} \geq 1500 \mathrm{rpm}$. No vortex was observed during the tests using the gear blank.

Figure 3.9 presents the same data as Figure 3.8, now as a function of $\bar{h}$ at discrete values of $\Omega_{r}$. A consistent difference between types of teeth (or lack thereof) is shown to be independent of $\bar{h}$. These results indicate clearly that a model that considers a disk with no teeth in place of a gear would underestimate drag losses by $10-50 \%$ depending on $\Omega_{r}$ and $\bar{h}$. Likewise, differences between spur and helical gears in terms of their impact on flow regime created within the gearbox indicate that use of a spur gear in place of a helical gear (i.e. a 2D CFD model in place of a 3D one) might involve errors in the range of $5-40 \%$. 

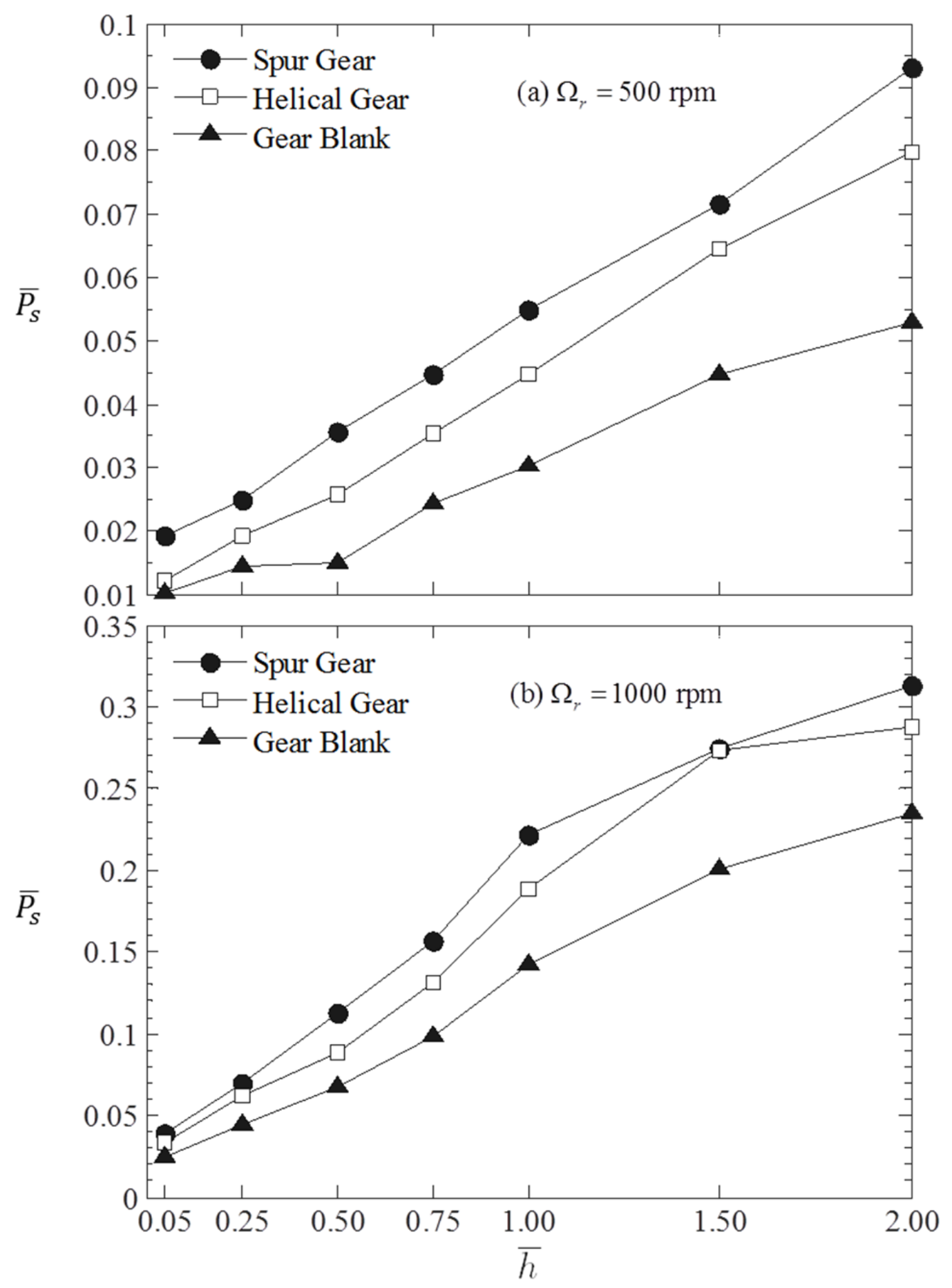

Figure 3.9 Effect of gear teeth on $\bar{P}_{s}$ within a range of $\bar{h}$ at (a) $\Omega_{r}=500 \mathrm{rpm}$, (b) $\Omega_{r}=1000 \mathrm{rpm},(\mathrm{c}) \Omega_{r}=1500 \mathrm{rpm}$, and (d) $\Omega_{r}=2000 \mathrm{rpm}$. (continued) 
Figure 3.9 (continued)
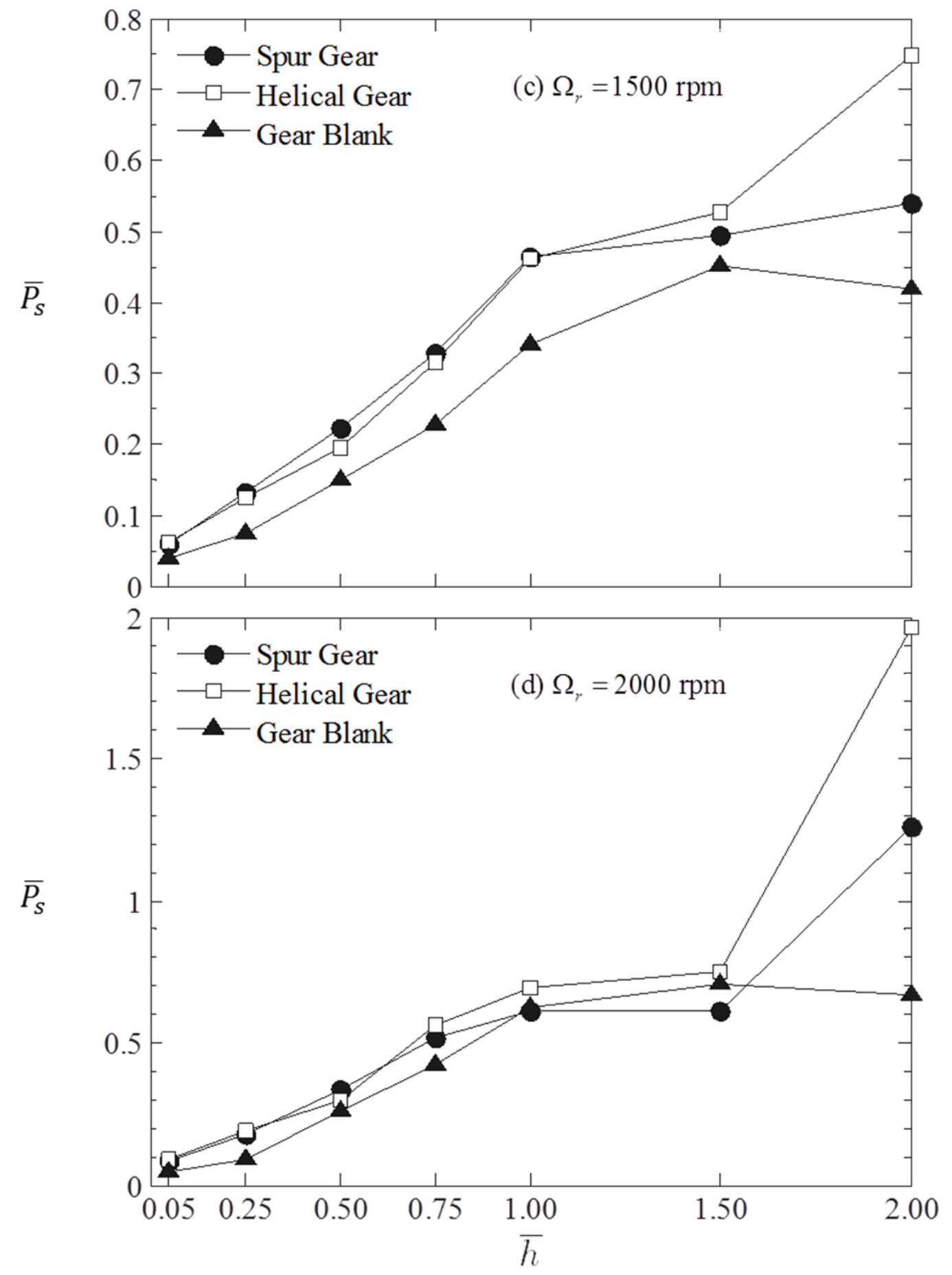


\subsection{Gear Pair Experiments}

The second group of tests defined in Table 2.2 considered a gear pair in the configuration shown in Figure 2.4(a) instead of a single ring gear. As such all the components of power losses defined in Eq. (2.1) including drag loss of the pinion, $P_{p d}$, and the gear mesh pocketing loss, $P_{p o c}$. Each subset of experiments defined in Table 2.2 was designed to quantify the effect of a certain parameter. Given the fact that the same tests were performed in previous sections for a single ring gear and bearings alone, these tests also provide means to break down $\bar{P}_{S}$ into its drag, bearing and pocketing components.

Formation of a vortex was shown to be more difficult in gear pair tests than a single ring gear test. The presence of the pinion in the path of oil was observed to provide some disturbance in the flow field and required the highest level of oil $(\bar{h}=2.00)$ and speed $\left(\Omega_{r}=2000 \mathrm{rpm}\right)$ to form a vortex in this gearbox. Additionally, as the pinion rotates in the opposite direction as the ring gear, a vortex in the direction of ring gear rotation is opposed by a vortex on the pinion, forcing the oil into the mesh.

\subsubsection{Effect of Gear Ratio}

Figure 3.10 shows the effect of gear ratio $n(n=4.563,4.250$ and 3.941 of gear pairs $\# 1$, $\# 2$ and \#3 in Table 2.1) on $\bar{P}_{S}$. In these tests, the pinion is placed directly on top of the ring gear at $\phi=90^{\circ}$. Here, the first two gear pairs with $n=4.563$ and 4.250 

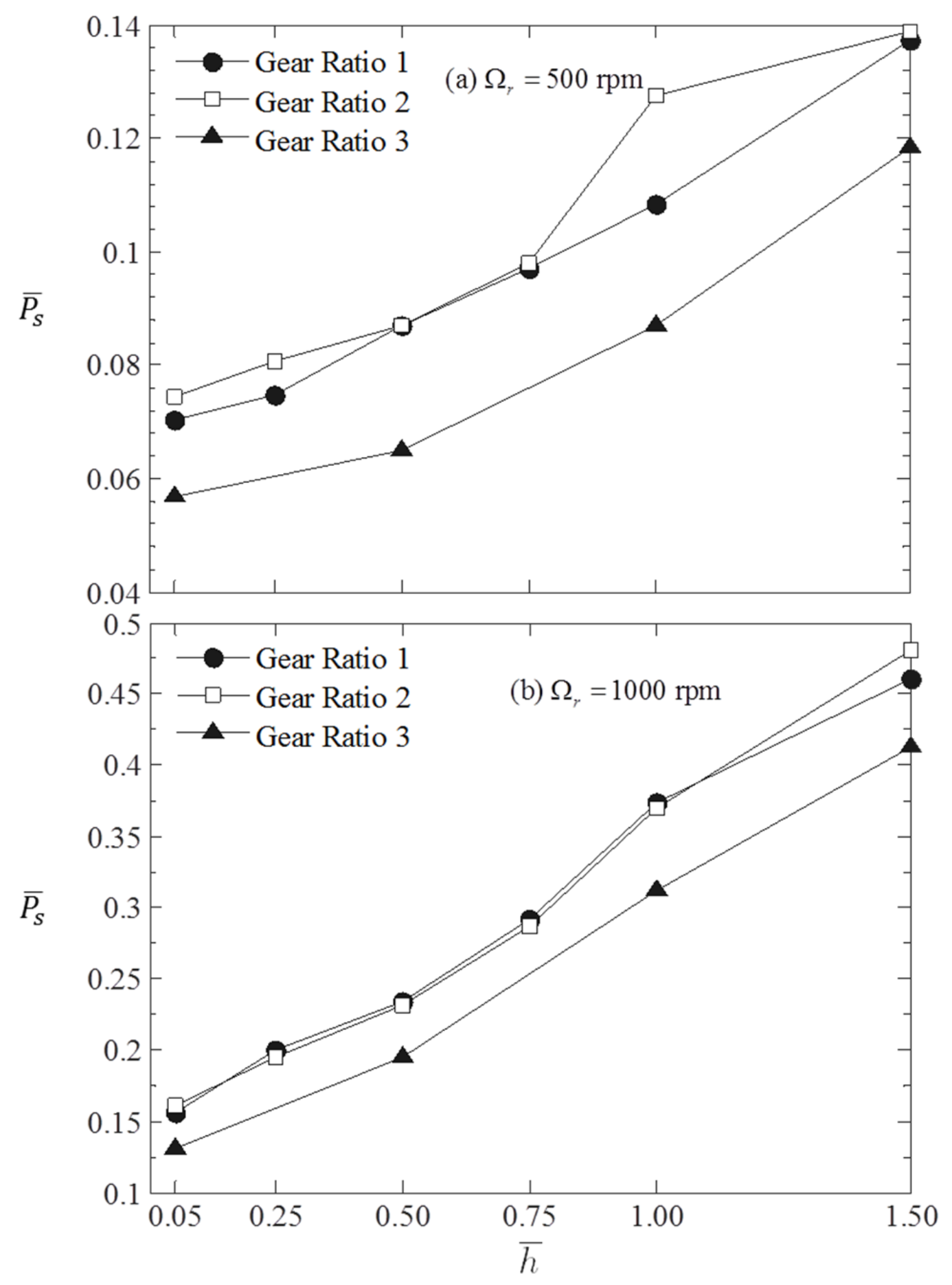

Figure 3.10 Influence of gear ratio $n$ on $\bar{P}_{s}$ within a range of $\bar{h}$ at (a) $\Omega_{r}=500 \mathrm{rpm}$, (b) $\Omega_{r}=1000 \mathrm{rpm}$, (c) $\Omega_{r}=1500 \mathrm{rpm}$, and (d) $\Omega_{r}=2000 \mathrm{rpm}$. Lubricant $\mathrm{B}$ at $90^{\circ} \mathrm{C}$ and $\phi=90^{\circ}$. (continued) 
Figure 3.10 (continued)
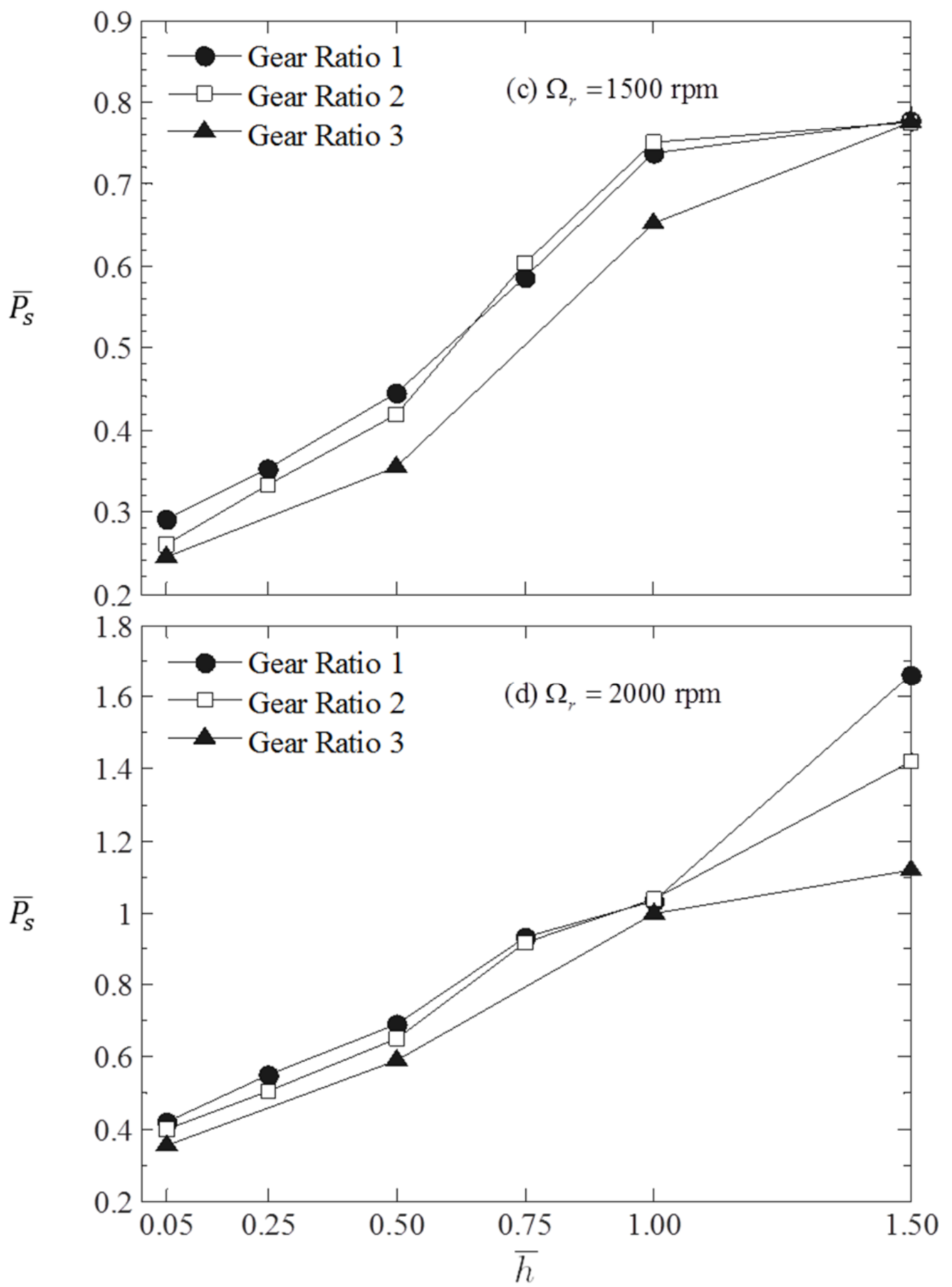
showed very little difference in $\bar{P}_{S}$ for all $\bar{h}$ and $\Omega_{r}$ values considered. The gear pair with the lowest ratio $(n=3.941)$ showed a measurable and consistently smaller $\bar{P}_{S}$ from the other two gear pairs. For instance, in Figure 3.10(b) for $\bar{h}=1.0$ and $\Omega_{r}=1000 \mathrm{rpm}$, the $\bar{P}_{S}$ values for $n=4.563$ and 4.250 are within $1 \%$ of each other while $\bar{P}_{S}$ of $n=3.941$ is nearly $20 \%$ lower than the other two ratios.

While this sub-study attempted to quantify differences between each gear ratio, it should be noted that other factors could have played a role in the test results such as the module of each gear pair (Table 2.1) or the pinion rotational speed. These factors were assumed negligible due to the similar results shown in Section 3.2.1, where ring gear diameter was shown to have a similar effect on $\bar{P}_{S}$ as $n$.

\subsubsection{Influence of Circumferential Pinion Position}

With the definition of Figure 2.9 for the position of the pinion in relation the ring gear, a family of tests were performed at different pinion position angles $\left(\phi \in\left[0^{\circ}, 180^{\circ}\right]\right)$ and static oil levels $(\bar{h} \in[0.05,2.0])$ with the oil level still measured from the bottom of the ring gear. Lubricant $\mathrm{B}$ at $90^{\circ} \mathrm{C}$ was used in these tests.

Figure 3.11 presents plots for $\bar{P}_{S}$ as a function of $\phi$ at different $\bar{h}$ and $\Omega_{r}$ values. It is observed that the influence of $\phi$ on $\bar{P}_{S}$ is very significant, especially at higher $\bar{h}$ values.

For instance, for $\bar{h}=2.00$ at $\Omega_{r}=1000 \mathrm{rpm} \quad$ (Figure 3.11(b)), $\bar{P}_{s}=1.51$ at 


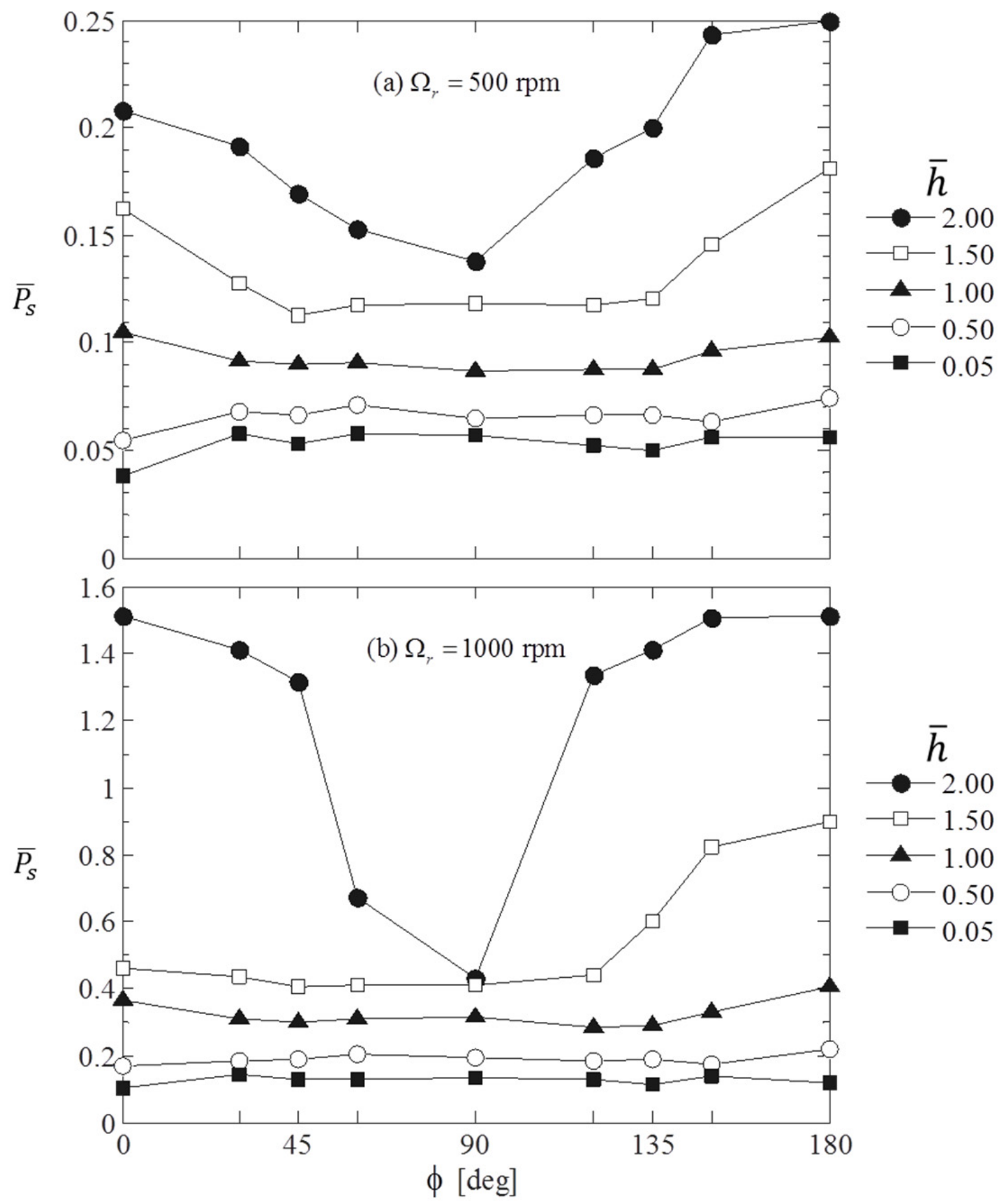

Figure 3.11 Effect of $\phi$ on $\bar{P}_{S}$ within a range of $\bar{h}$ at (a) $\Omega_{r}=500 \mathrm{rpm}$, (b) $\Omega_{r}=1000$ $\mathrm{rpm}$, (c) $\Omega_{r}=1500 \mathrm{rpm}$, and (d) $\Omega_{r}=2000 \mathrm{rpm}$. Lubricant B at $90^{\circ} \mathrm{C}$. (continued) 
Figure 3.11 (continued)
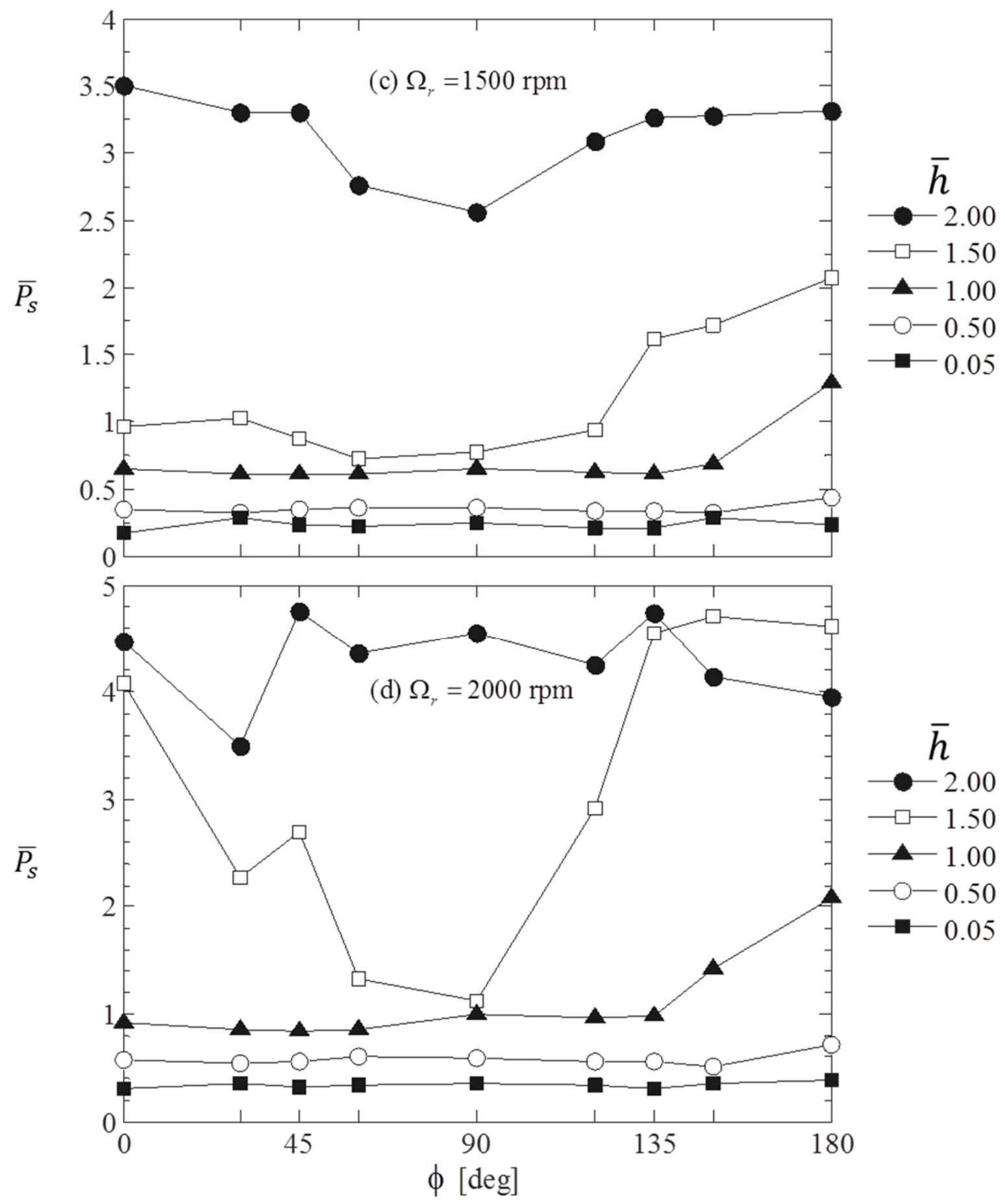
$\phi=0^{\circ}$ and $180^{\circ}$ (i.e. when the pinion center is at the same elevation as the ring gear) while $\bar{P}_{S}=0.43$ for $\phi=90^{\circ}$, representing more than a threefold difference. For $\bar{h} \geq 1.5$, $\bar{P}_{S}$ is at a minimum when $\phi=90^{\circ}$ while it is maximum is at $\phi=180^{\circ}$. This is simply due to the relative position of the pinion with respect to the static oil level. When $\phi=90^{\circ}$, the gear mesh is at the highest possible point and the least amount of oil is likely to be forced to the gear mesh even with the highest $\bar{h}$ values. Meanwhile when $\phi=0^{\circ}$ and $180^{\circ}$ with $\bar{h} \geq 1.5$, the gear mesh is completely below the static oil level such that pumping losses at the gear mesh are maximized. In support of this argument, the influence of $\phi$ on $\bar{P}_{S}$ is seen to diminish at lower $\bar{h}$ values. In Figure 3.11(b), for instance, $\bar{P}_{S}$ remains relatively unchanged with $\phi$ for $\bar{h}<1.0$.

Furthermore, $\bar{P}_{S}$ is higher for $\bar{h} \geq 1.0$ when $\phi=180^{\circ}$ (up-mesh) than when $\phi=0^{\circ}$ (down-mesh). The reason is due to the direction of rotation of the ring gear, as seen schematically in Figure 2.9. The inlet to the gear mesh is down when $\phi=0^{\circ}$ indicating that the gear mesh is filled with a mixture of oil and air while the inlet to the mesh is up when $\phi=180^{\circ}$ indicating that a high concentration of oil is pulled in. In addition, observed operating oil levels before any vortex is formed indicated that the effective oil level at the $\phi=0^{\circ}$ side was lower than that of the other side as depicted schematically in Figure 3.12. These up-mesh versus down-mesh effects are best seen in Figures 3.11(c) 
and $3.11(\mathrm{~d})$ at $\bar{h}=1.00$ where $\bar{P}_{S}$ is $125 \%$ higher in the up-mesh $\left(\phi=180^{\circ}\right)$ condition than in the down-mesh $\left(\phi=0^{\circ}\right)$ condition.

Formation of a vortex was observed to be easier when the when the mesh was submerged in oil although the presence of the pinion delayed vortex formation compared to a single ring gear. The scatter in the data at $\Omega_{r}=2000 \mathrm{rpm}$ and $\bar{h}=2.0$ in Figure 3.11(d) is a direct result of vortex formation.

\subsubsection{Components of Spin Power Loss}

Gear pair tests presented thus far in Section 3.3 will be combined with single ring gear tests from Section 3.2 and bearing/seal tests from Section 3.1.1 to quantify the contributions of power loss components to total spin loss $\bar{P}_{S}$. Equation (2.1) is repeated here with losses associated with each gear and bearing-seal set separated:

$$
P_{s}=P_{r d}+P_{p d}+P_{p o c}+\left[P_{b}+P_{\text {seal }}\right]_{p}+\left[P_{b}+P_{\text {seal }}\right]_{r}
$$

The power loss $\bar{P}_{S}$ values presented in Sections 3.3.1 and 3.3.2 included all components of gear pair spin loss. The bearing and seal power loss of a shaft was measured first without gears as presented in Figure 3.2. In that data, ring gear shaft bearings and seal loss, $\left[P_{b}+P_{\text {seal }}\right]_{r}$, is the value of loss at $\Omega_{r}$ and the pinion bearings and seal loss, $\left[P_{b}+P_{\text {seal }}\right]_{p}$, is the value at $n \Omega_{r}$. 
The ring gear drag loss, $P_{r d}$, is calculated by subtracting the $\left[P_{b}+P_{\text {seal }}\right]_{r}$ value for the ring gear shaft bearings and seal from single ring gear losses given in Section 3.2.1. Finally, assuming that the drag loss of the pinion $P_{p d}$ is negligible as the pinion diameter is substantially smaller than the ring gear, $P_{p o c}$ is isolated as the only unknown parameter in Eq. (3.1). In other words, $P_{p o c}$ is quantified by comparing results of four separate tests: bearing-seal loss tests at $\Omega_{r}$ and $n \Omega_{r}$, single ring gear tests at $\Omega_{r}$ and gear pair tests at $\Omega_{r}$.

The contributions of spin power loss are shown in Figures 3.12 to 3.14 for various conditions. Here tests of gear pair $\# 3$ with $n=3.941$ (lubricant B at $60^{\circ} \mathrm{C}$ ) are used. Figure 3.12 shows pinion bearings $\left(\left[P_{b}+P_{\text {seal }}\right]_{p}\right)$, ring gear bearings $\left(\left[P_{b}+P_{\text {seal }}\right]_{r}\right)$, ring gear drag $\left(P_{r d}\right)$, and gear mesh pocketing $\left(P_{p o c}\right)$ components for $\phi=0^{\circ}$ (downmesh), adding up to $\bar{P}_{S}$. The contribution of $P_{p o c}$ is seen to be negligible for $\bar{h}=0.5$ and 1.0 in Figures 3.12(a) and (b), respectively, when the mesh is pulling down mostly air. However, as the gear mesh becomes submersed in oil, $P_{p o c}$ component becomes more significant as shown in Figures 3.12(c) and (d). In Figure 3.12(d) for $\bar{h}=2.0$ at $\Omega_{r}=1000 \mathrm{rpm}$, the pocketing component contributed $62 \%$ of $\bar{P}_{s}$, while $P_{r d}$ is responsible for $18 \%$, and $\left[P_{b}+P_{\text {seal }}\right]_{p}$ and $\left[P_{b}+P_{\text {seal }}\right]_{r}$ constitute the remaining $20 \%$. 

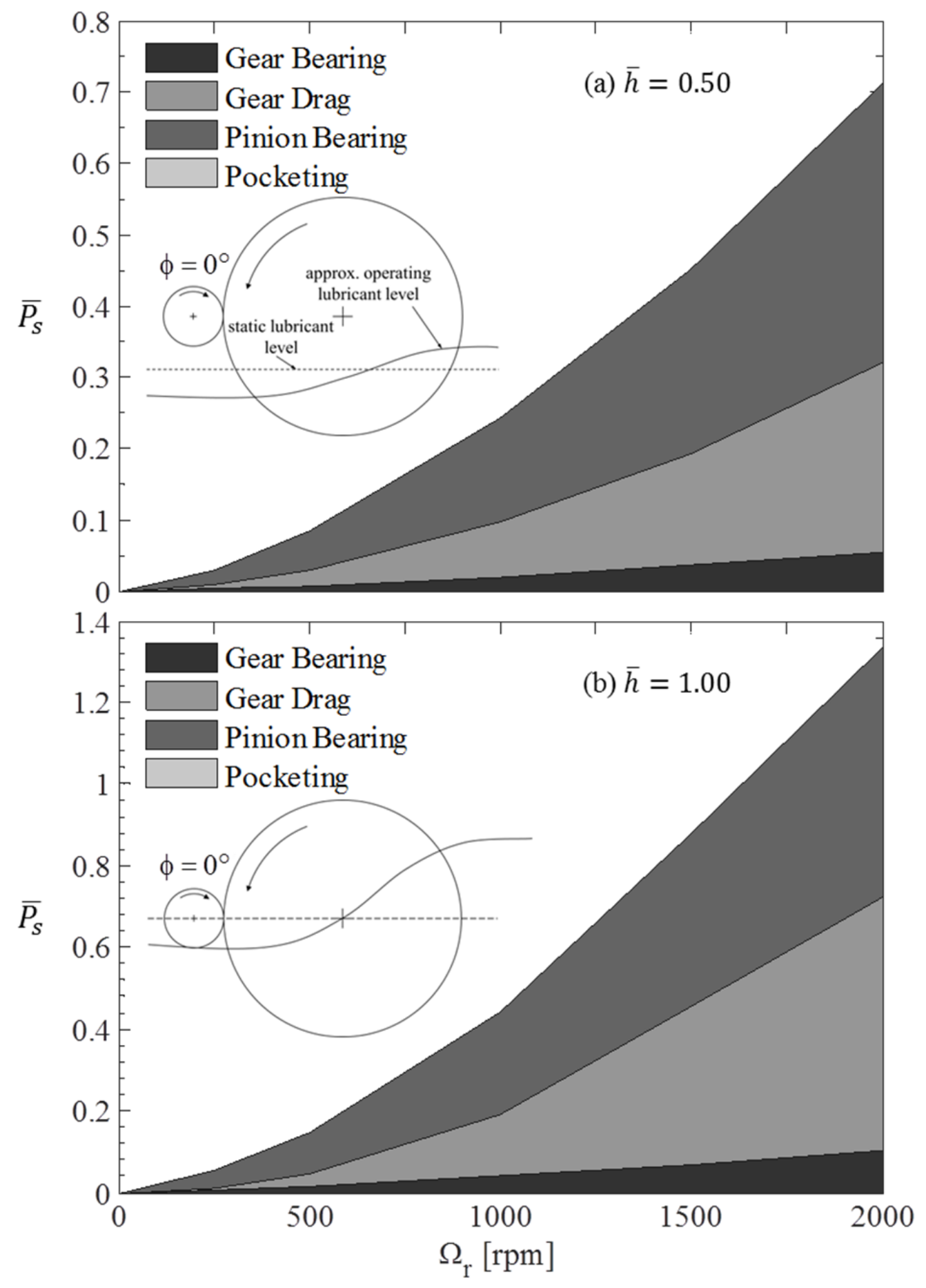

Figure 3.12 Components of $\bar{P}_{S}$ with gear pair \#3 and the pinion at $\phi=0^{\circ}$ for (a) $\bar{h}=0.5$, (b) $\bar{h}=1.0$, (c) $\bar{h}=1.5$, and (d) $\bar{h}=2.0$. Lubricant $\mathrm{B}$ at $60^{\circ} \mathrm{C}$. (continued) 
Figure 3.12 (continued)
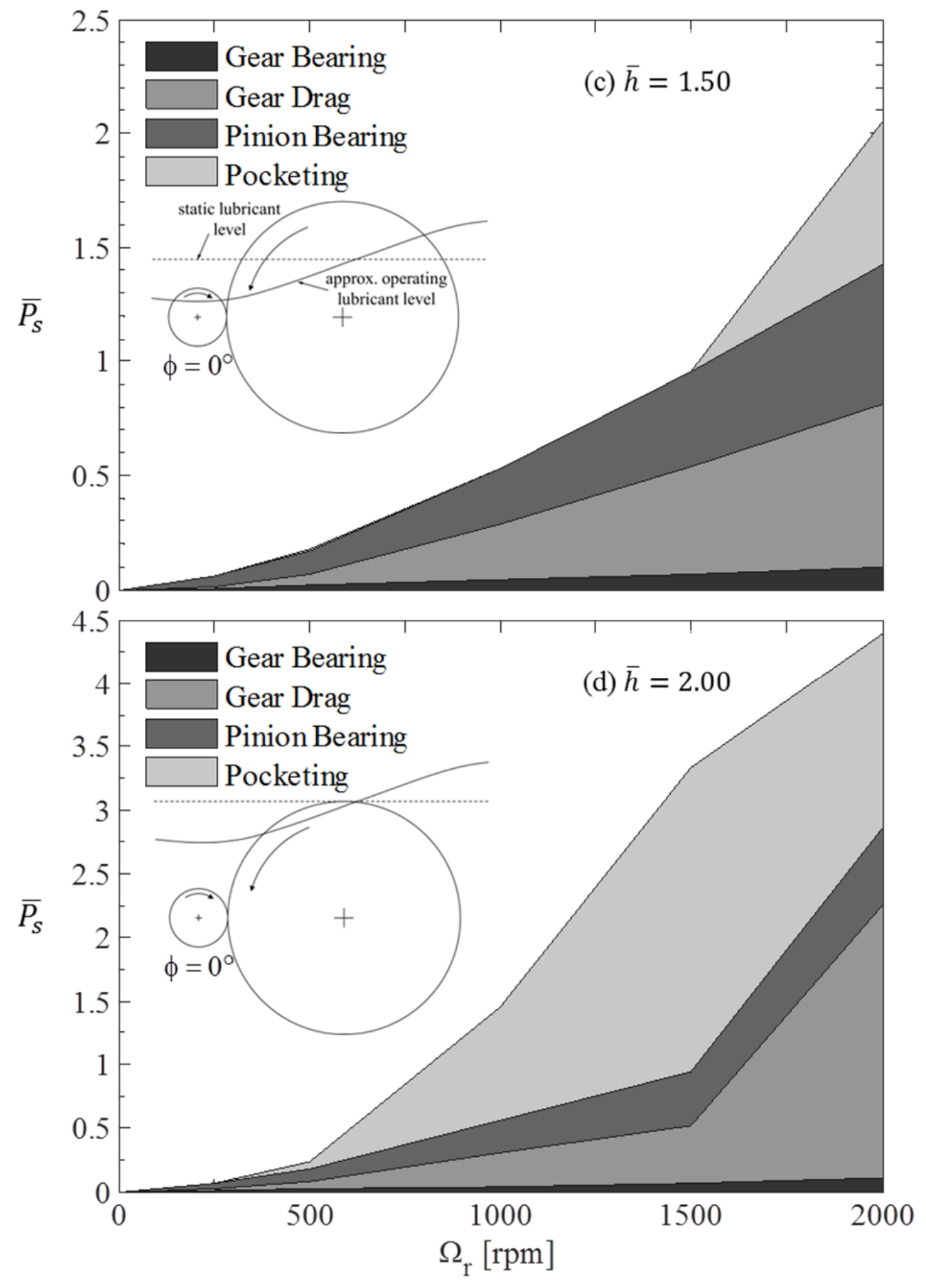

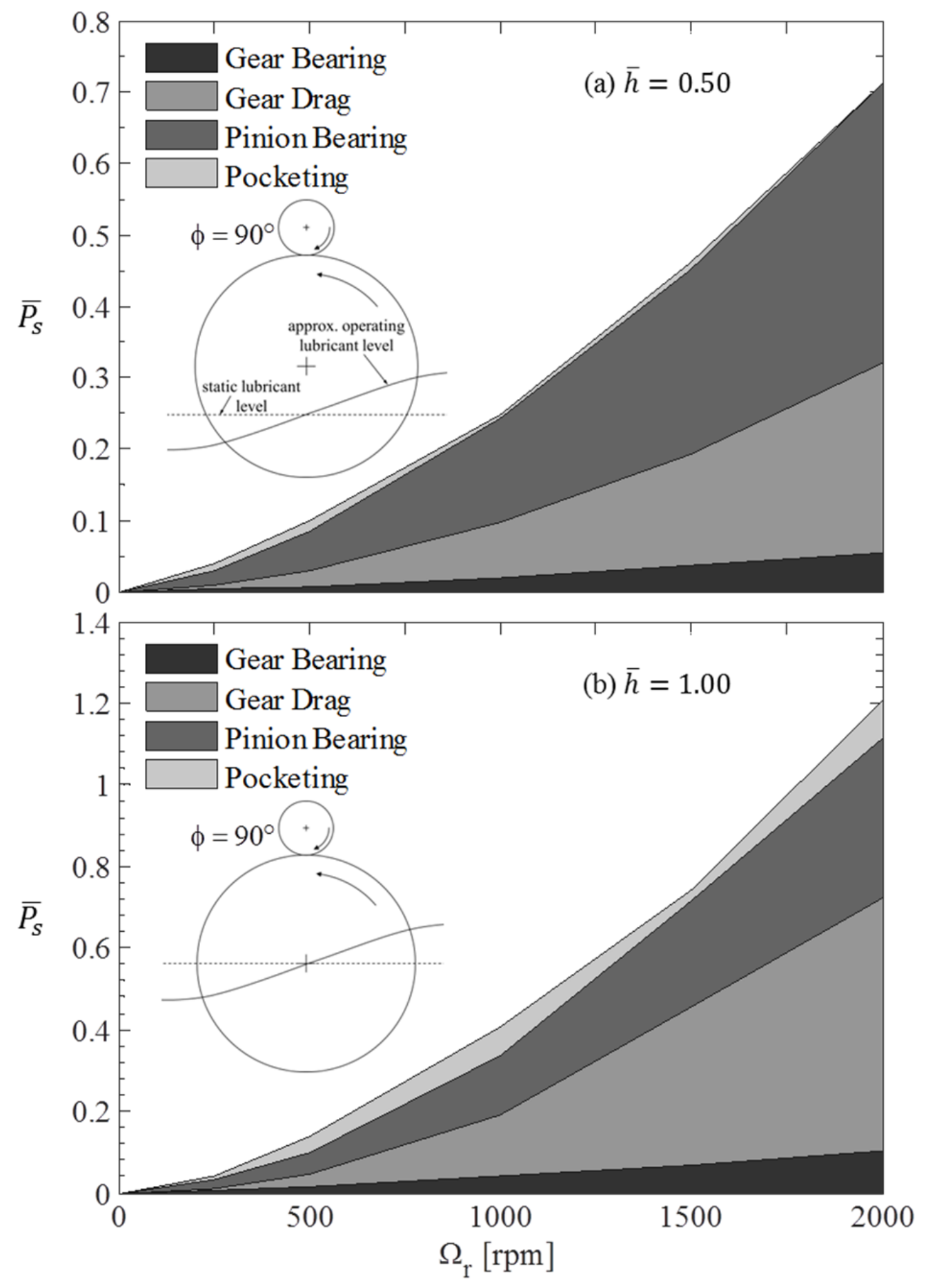

Figure 3.13 Components of $\bar{P}_{S}$ with gear pair \#3 and the pinion at $\phi=90^{\circ}$ for (a) $\bar{h}=0.5$, (b) $\bar{h}=1.0$, (c) $\bar{h}=1.5$, and (d) $\bar{h}=2.0$. Lubricant B at $60^{\circ} \mathrm{C}$. (continued) 
Figure 3.13 (continued)
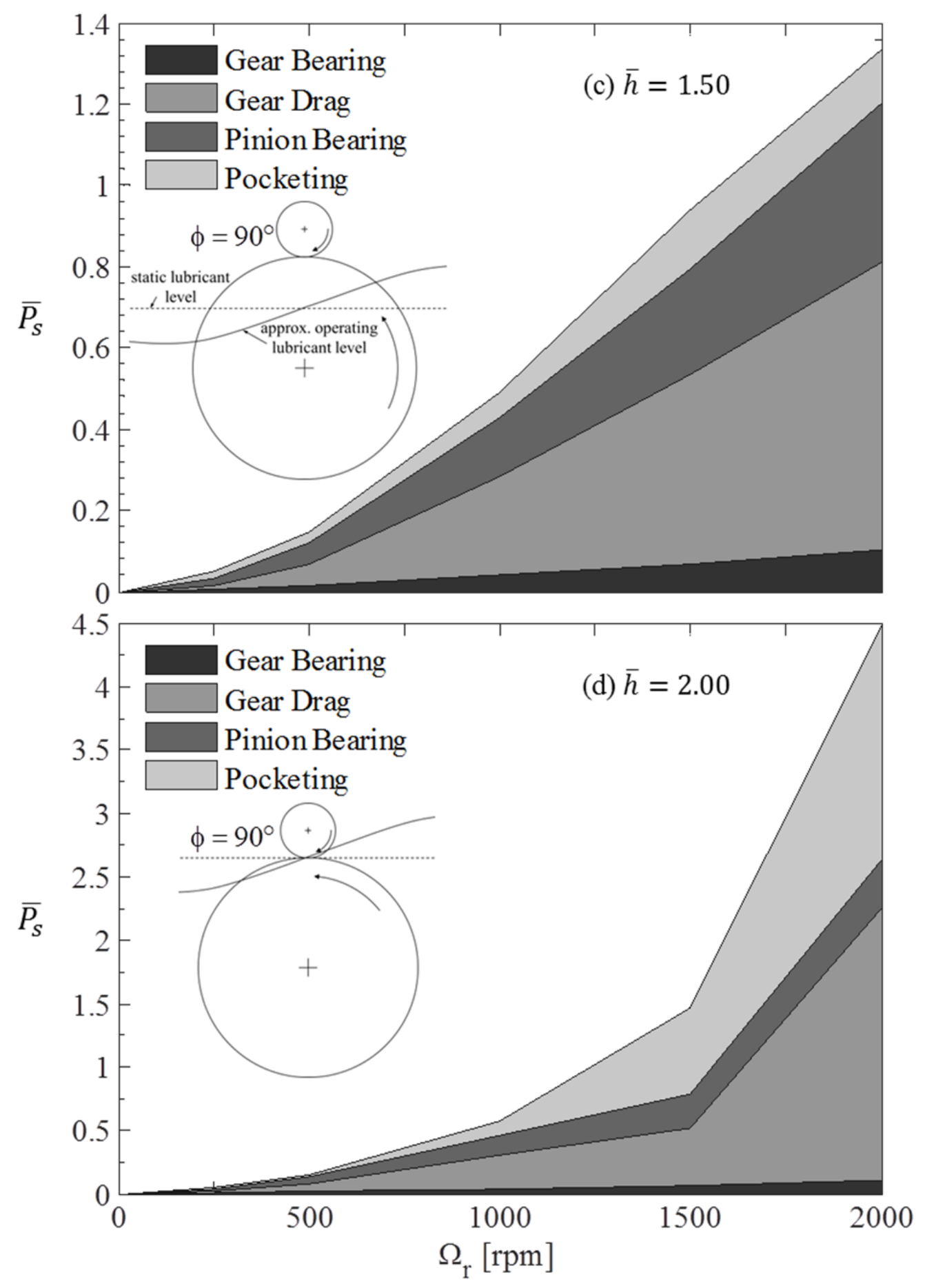

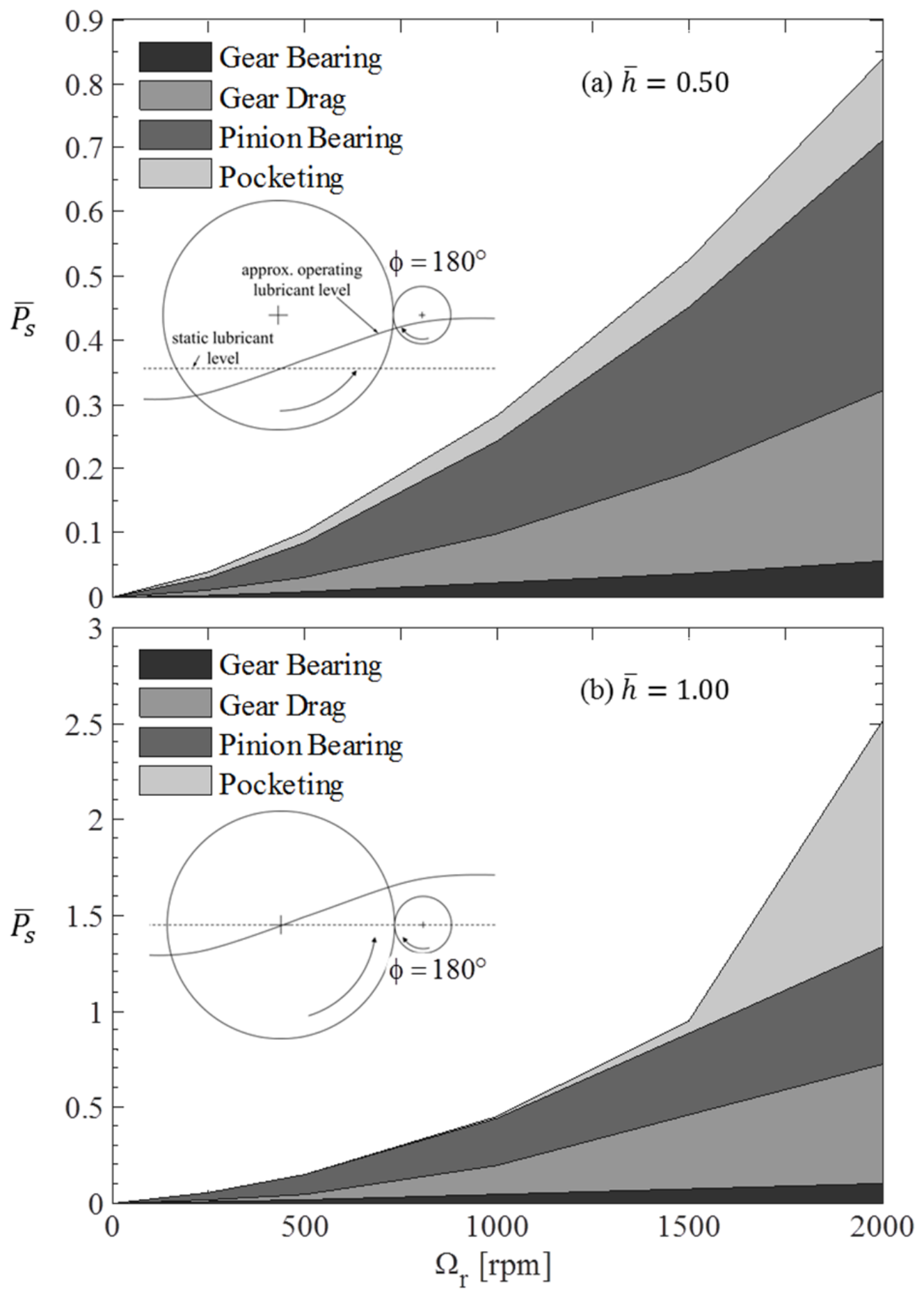

Figure 3.14 Components of $\bar{P}_{S}$ with gear pair \#3 with the pinion at $\phi=180^{\circ}$ for (a) $\bar{h}=0.5$, (b) $\bar{h}=1.0$, (c) $\bar{h}=1.5$, and (d) $\bar{h}=2.0$. Lubricant B at $60^{\circ} \mathrm{C}$. (continued) 
Figure 3.14 (continued)
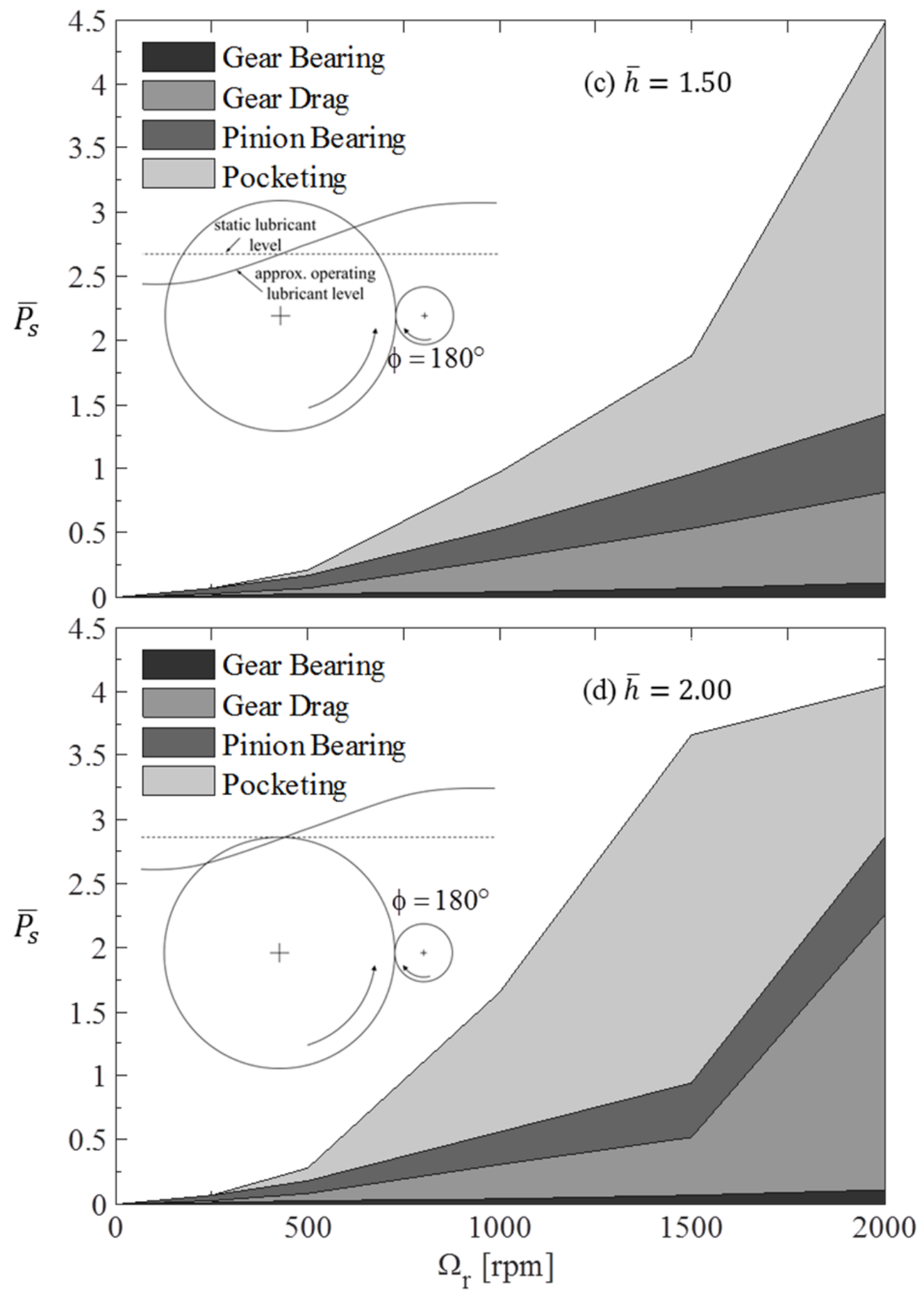
Figure 3.13 shows the same data for $\phi=90^{\circ}$. While the gear mesh is at its highest possible position in relation to oil level, the effective density of the medium pumped into the mesh should be higher than the $\phi=0^{\circ}$ position when the mesh is not submerged. This corresponds to the circumferential distance the lubricant must travel to reach the gear mesh. Accordingly, the $P_{p o c}$ component of the total spin loss is slightly more when $\bar{h}=0.50$ and 1.00 than those in Figure 3.12(a) and (b). $P_{p o c}$ is responsible for $8 \%$ of the losses at $\Omega_{r}=2000 \mathrm{rpm}$ when $\bar{h}=1.0$. When $\bar{h}=2.0$ (the ring gear is fully submerged) as shown in Figure 3.13(d), the gear mesh is fully submersed in oil. At $\Omega_{r}=1000 \mathrm{rpm}, P_{p o c}$ constituted only $21 \%$ of $\bar{P}_{S}$, while $P_{r d}$ is responsible for $47 \%$, and bearing and seal losses are $32 \%$.

Finally, Figure 3.14 presents the corresponding results for $\phi=180^{\circ}$ (up-mesh). It is safe to state that the oil/air ratio of the medium going into the gear mesh is the highest in this pinion position. Even at low submersion levels, such as in Figure 3.14(a) and (b), the contribution of $P_{p o c}$ is shown to be larger when compared with respective plots from Figure 3.12 and Figure 3.13. Illustrated in Figure 3.14(d) at $\Omega_{r}=1000 \mathrm{rpm}$ and $\bar{h}=2.0$, $P_{p o c}, P_{r d}$ and bearing-seal components represent $66 \%, 16 \%$, and $18 \%$ of the total loss, respectively.

Additionally, noteworthy are the similarities between Figure 3.12(d) and Figure 3.14(d). These correspond to $\phi=0^{\circ}$ and $\phi=180^{\circ}$, respectively for $\bar{h}=2.0$. With these 
conditions, it would be reasonable to assume that the density of the oil/air mixture forced into mesh is the very similar for each case.

\subsection{Results of Enclosure Experiments}

This section presents results of spin power loss experiments performed using side and peripheral enclosures, as described in Chapter 2 and pictured in Figure 2.12. The tests presented in this section were presented in the test matrix described in Table 2.2(a). Three important aspects of these tests should be noted as follows:

(i) Significantly reduced volume of oil within the gearbox. The majority of empty space around the gear when operating with no enclosure was filled by polycarbonate enclosure pieces. This dramatically reduced the volume of oil required to achieve various static lubricant levels. Therefore, the inertial effects between the no enclosure studies and studies in this section are quite different.

(ii) Fluid flow behavior. The shape of the enclosures allowed for accumulation of oil in the large space above the gear to better simulate conditions in an automotive transmission. This greatly affected the variation between static lubricant levels and observed operating lubricant levels. Instead of the gear rotating through the static oil level as it does without enclosures, the motion of the gear ejected most of the oil and a small oil pool was left in the bottom of the housing, as shown in Figure 3.15. 


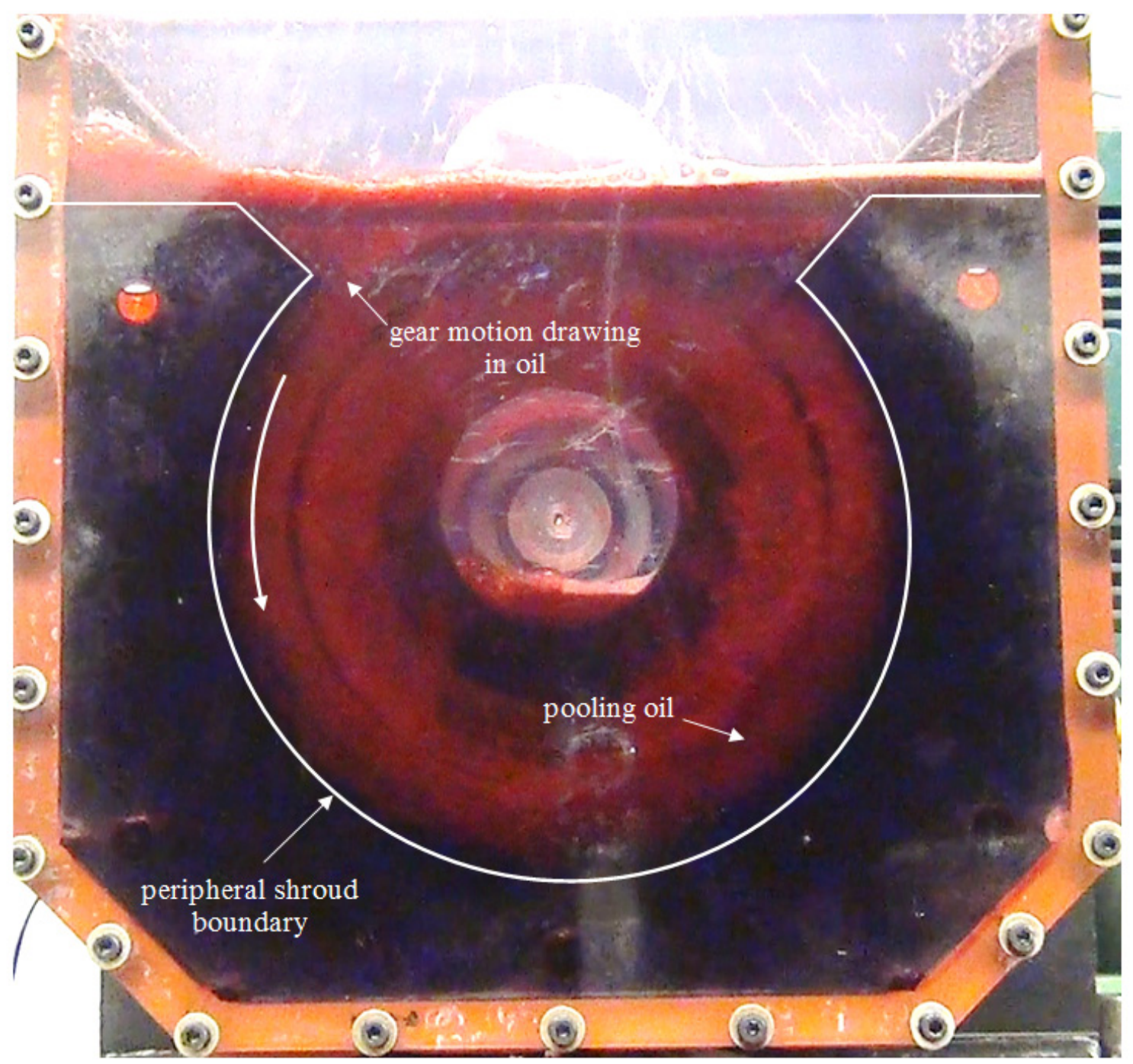

Figure 3.15 An example lubricant flow behavior in an enclosed environment $(\bar{h}=1.0$ and $\left.\Omega_{r}=1000 \mathrm{rpm}\right)$. 
(iii) No vortex development in tests with enclosures. Section 3.1.2 described conditions that typically resulted in a vortex formation under no enclosure conditions. Tests described in this section never experienced such vortex development.

Figures 3.16 and 3.17 show the effect of peripheral gap, $p$, on $\bar{P}_{S}$ for axial gaps of $s=12$ and $1 \mathrm{~mm}$, respectively. For both $s$ values, the $\bar{P}_{s}$ values corresponding to $p=5$ and $12 \mathrm{~mm}$ are rather close for $\Omega_{r} \leq 1000 \mathrm{rpm}$ while diverging slightly at higher speeds. However the differences are not significant, indicating that peripheral enclosures within these ranges are not very different in terms of $\bar{P}_{S}$.

Figure 3.18 and 3.19 show the effect of axial clearance, $s$, on $\bar{P}_{S}$ for constant peripheral gaps of $p=12$ and $5 \mathrm{~mm}$, respectively. Regardless of $p$, the value of $s$ is seen to be rather influential on $\bar{P}_{S}$ especially at higher $\bar{h}$ conditions. Both figures show the same trend in the effects of $s$ on $\bar{P}_{s}$. Figure 3.18(d) for $p=12$ and $\Omega_{r}=2000 \mathrm{rpm}$ shows a $62 \%$ reduction in $\bar{P}_{S}$ when the axial gap is reduced from $s=12 \mathrm{~mm}$ to $s=1$ $\mathrm{mm}$ at $\bar{h}=2.0$. These results in general suggest that $\bar{P}_{S}$ is lowest for the smallest $s$.

Figure 3.20 illustrates the dramatic effect of enclosures on $\bar{P}_{s}$. This figure combines the data from tests with enclosures ( $p=5 \mathrm{~mm}$ and $s \in[1,12] \mathrm{mm}$ ) and tests with no enclosures. These results show that even a modest enclosure with $p=5 \mathrm{~mm}$ and $s=12 \mathrm{~mm}$ reduces $\bar{P}_{s}$ by $53 \%$ from the no-enclosure condition at $\bar{h}=2.00$ and $\Omega_{r}=1000 \mathrm{rpm}$. 

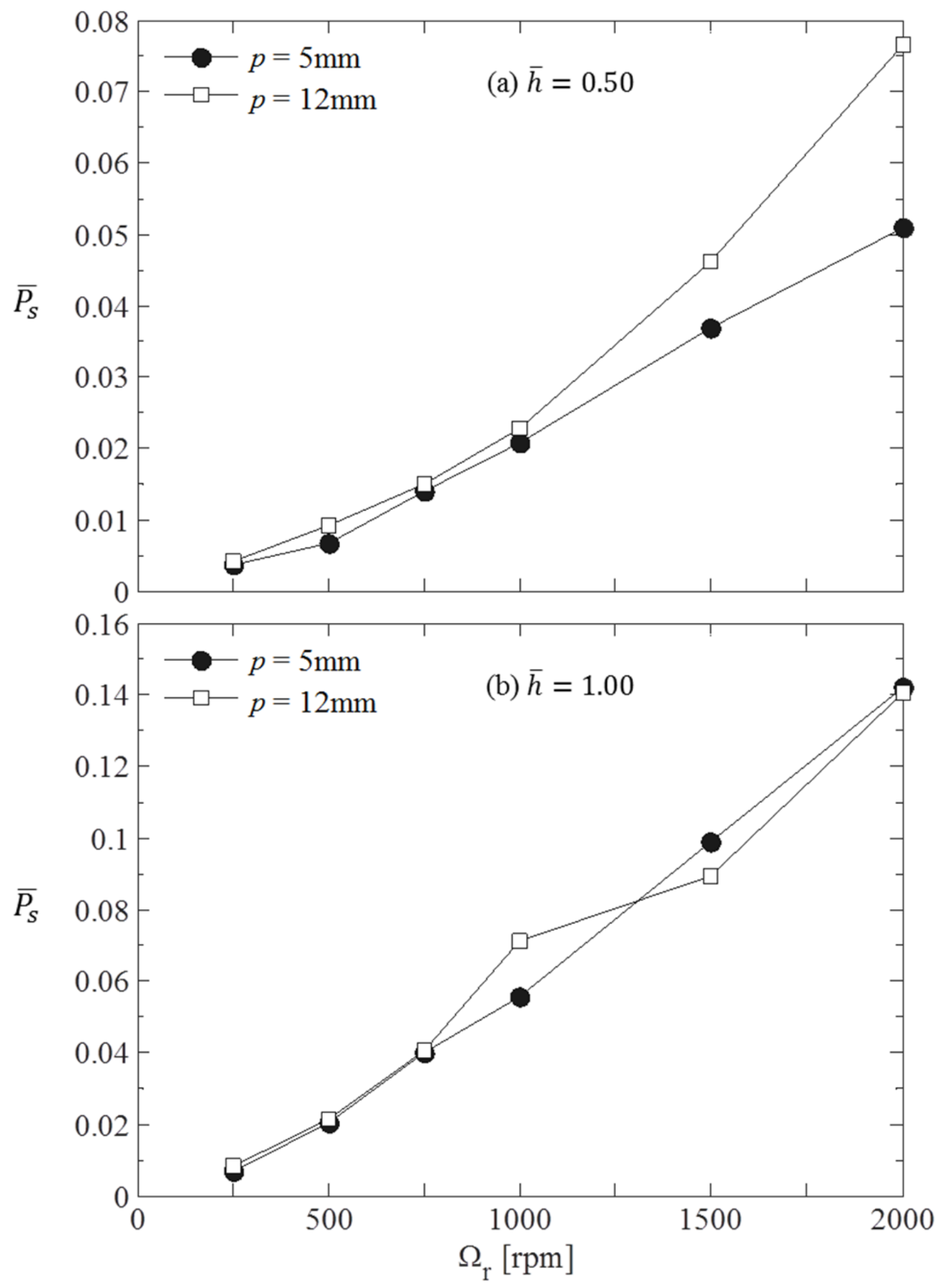

Figure 3.16 Effect of $p$ on $\bar{P}_{s}$ from a ring gear test with $s=12 \mathrm{~mm}$ at (a) $\bar{h}=0.5$, (b) $\bar{h}=1.0$, (c) $\bar{h}=1.5$, and (d) $\bar{h}=2.0$. Ring gear $\# 3$ with lubricant B at $60^{\circ} \mathrm{C}$. (continued) 
Figure 3.16 (continued)

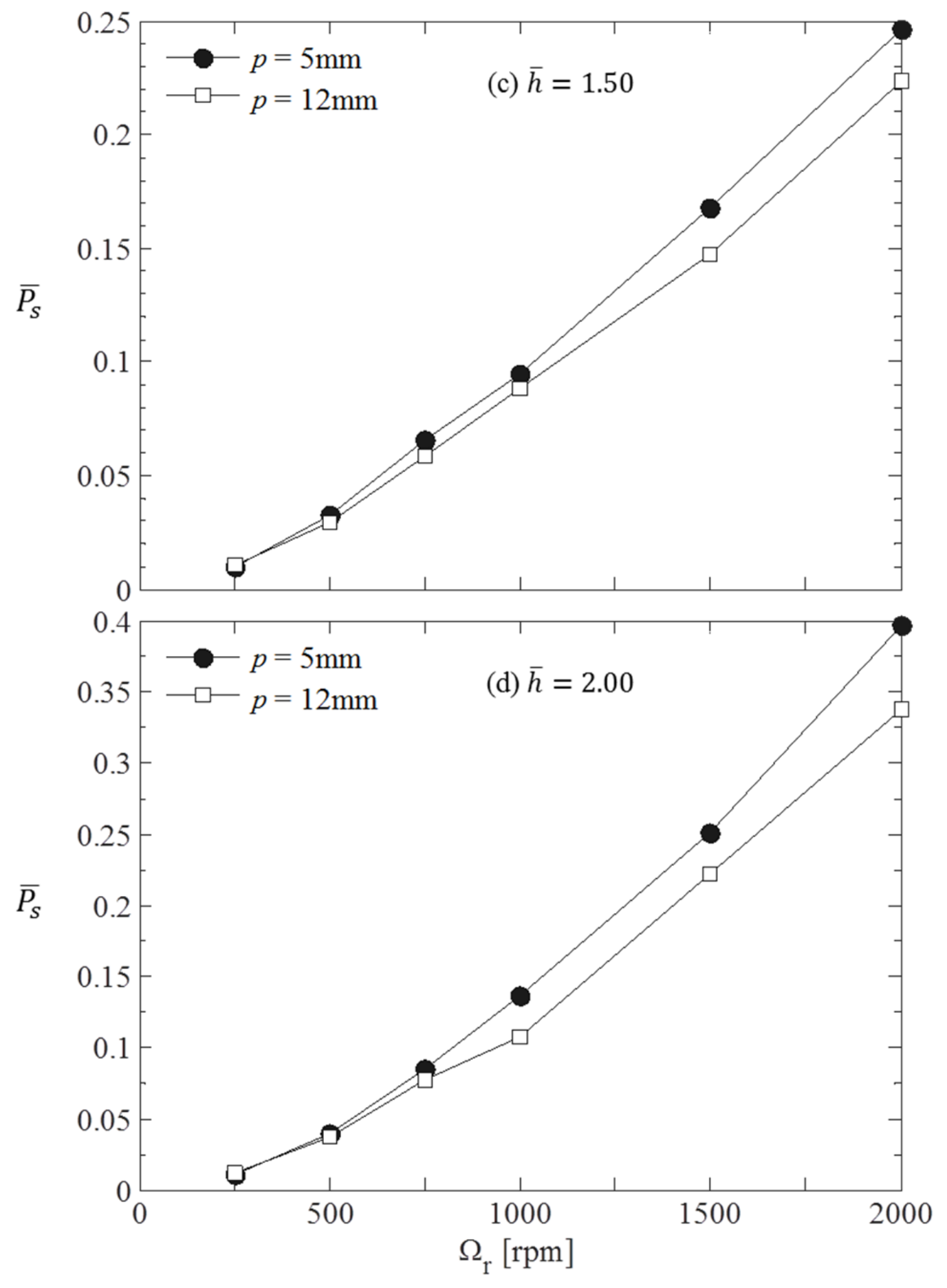



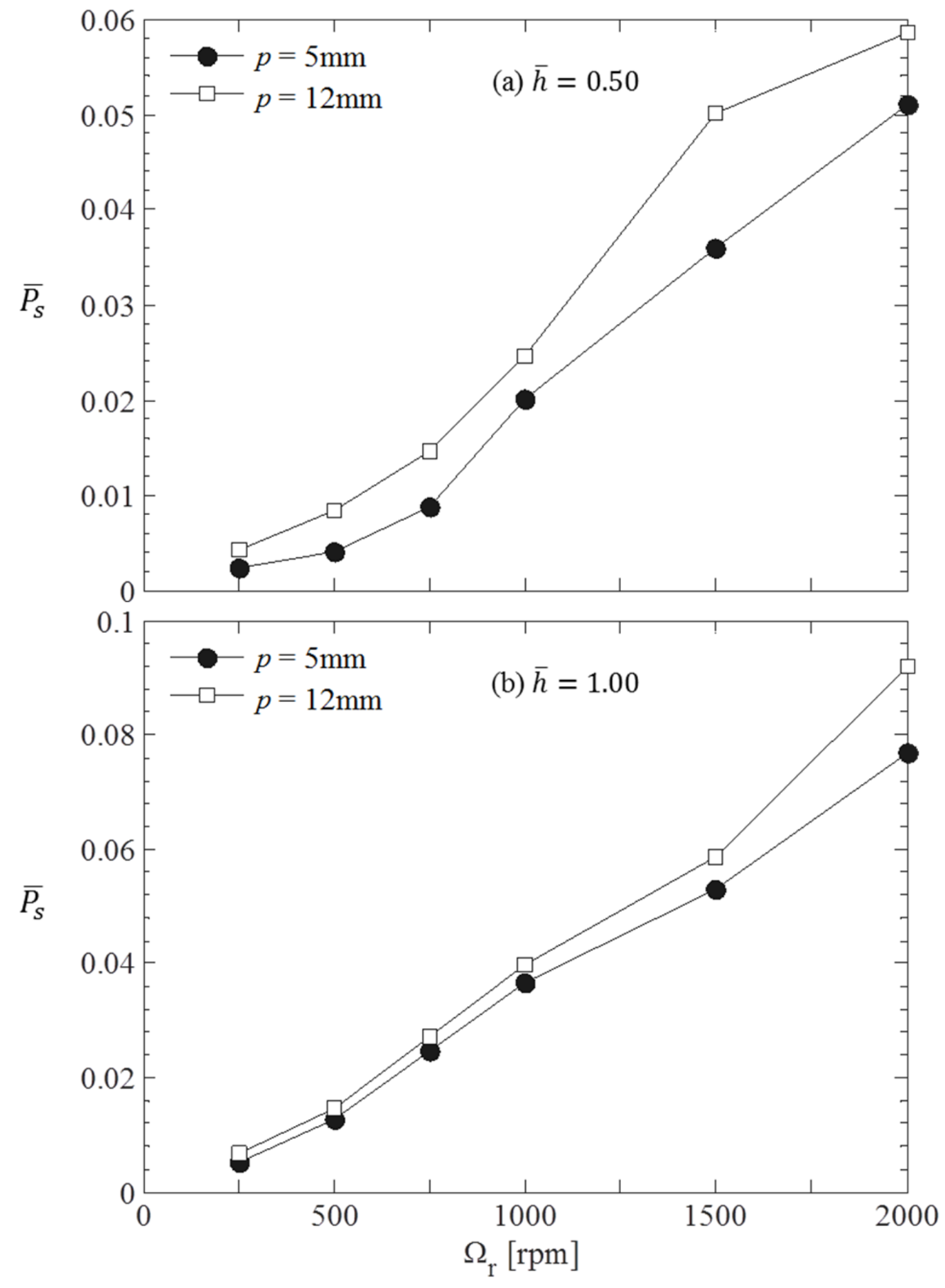

Figure 3.17 Effect of $p$ on $\bar{P}_{s}$ from a ring gear test with $s=1 \mathrm{~mm}$ at (a) $\bar{h}=0.5$, (b) $\bar{h}=1.0$, (c) $\bar{h}=1.5$, and (d) $\bar{h}=2.0$. Ring gear \#3 with lubricant B at $60^{\circ} \mathrm{C}$. (continued) 
Figure 3.17 (continued)
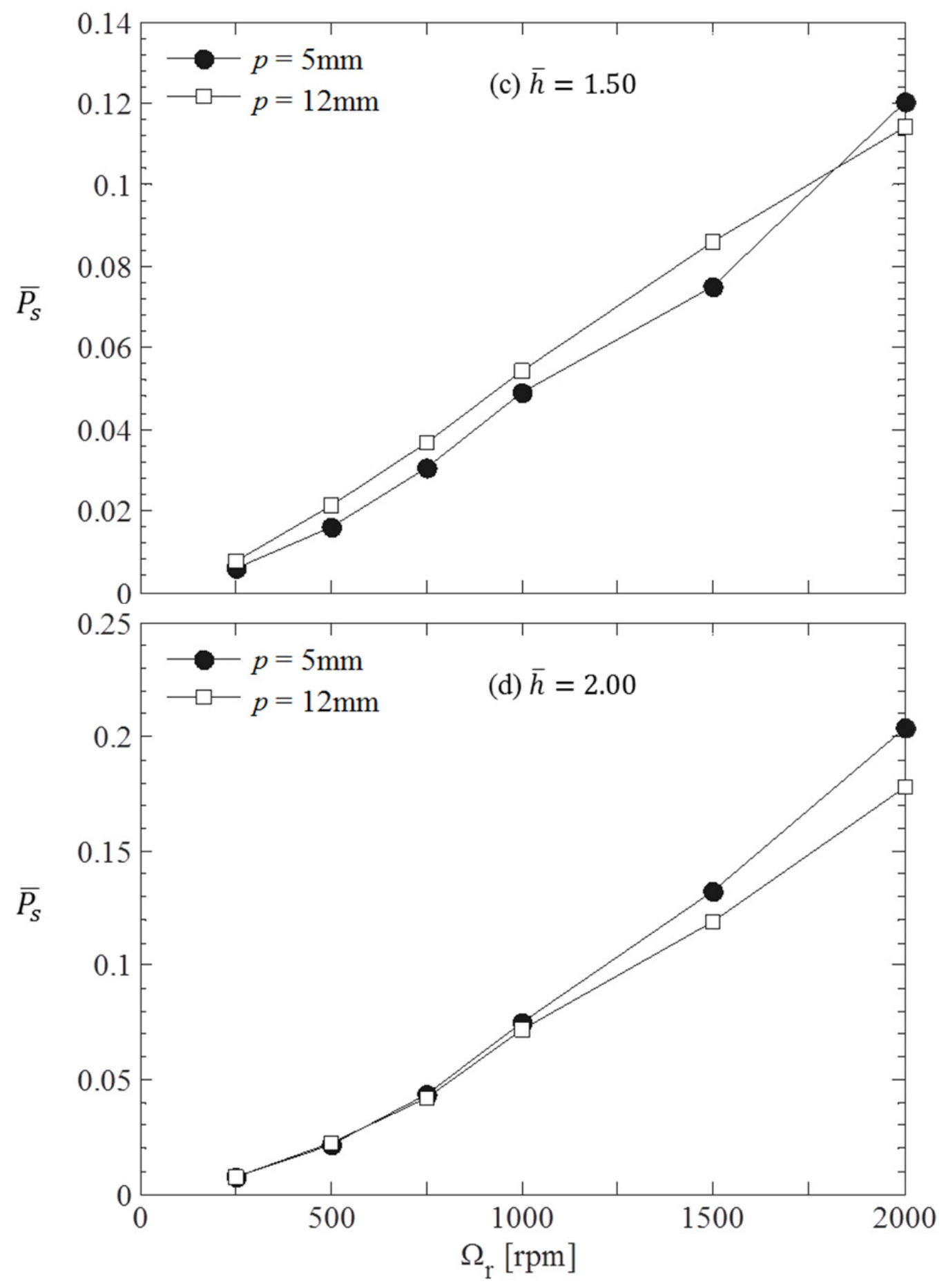


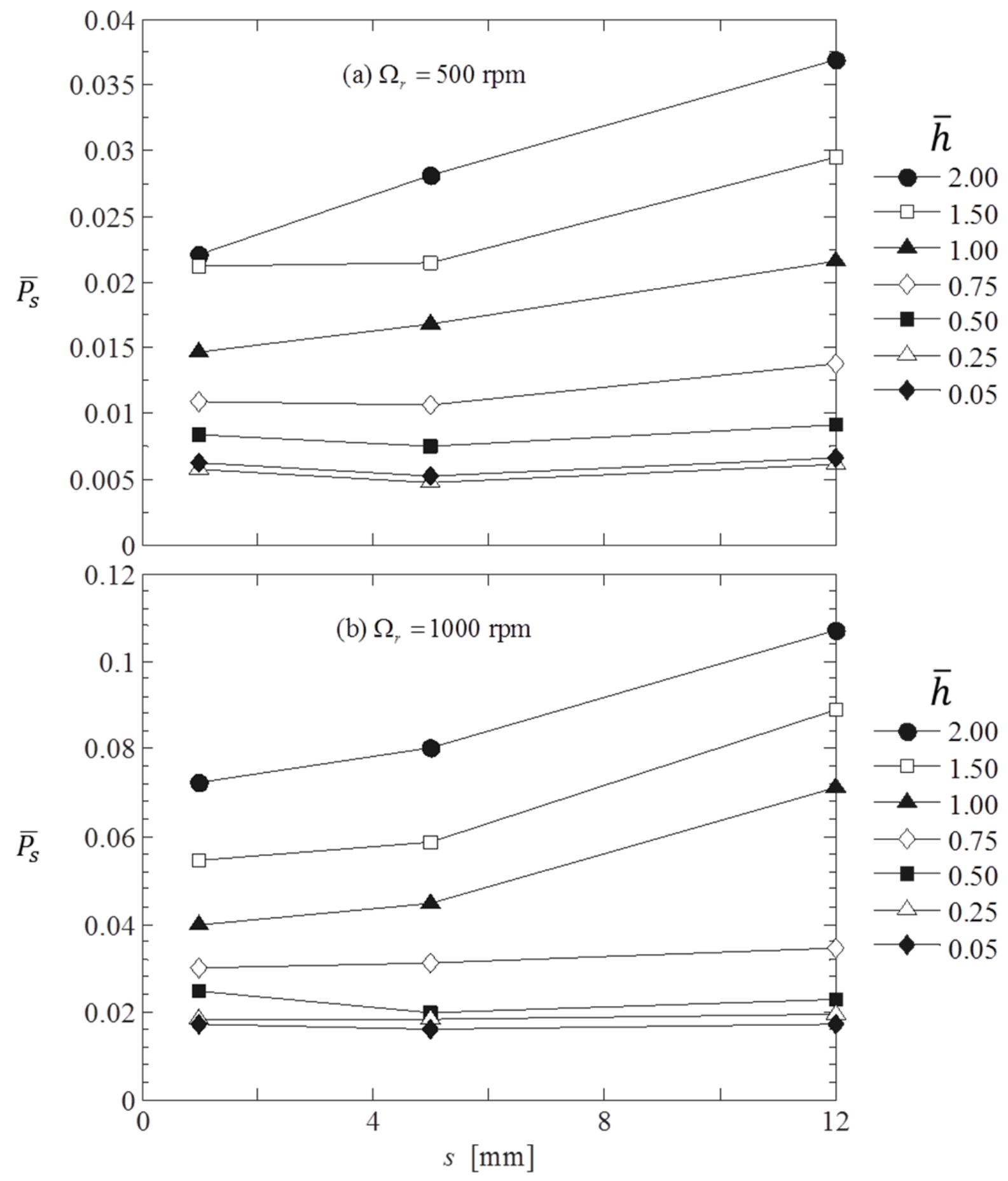

Figure 3.18 Effect of $s$ on $\bar{P}_{s}$ from a ring gear test with $p=12 \mathrm{~mm}$ at (a) $\Omega_{r}=500$ rpm, (b) $\Omega_{r}=1000 \mathrm{rpm}$, (c) $\Omega_{r}=1500 \mathrm{rpm}$, and (d) $\Omega_{r}=2000 \mathrm{rpm}$. Ring gear \#3 with lubricant $\mathrm{B}$ at $60^{\circ} \mathrm{C}$. (continued) 
Figure 3.18 (continued)

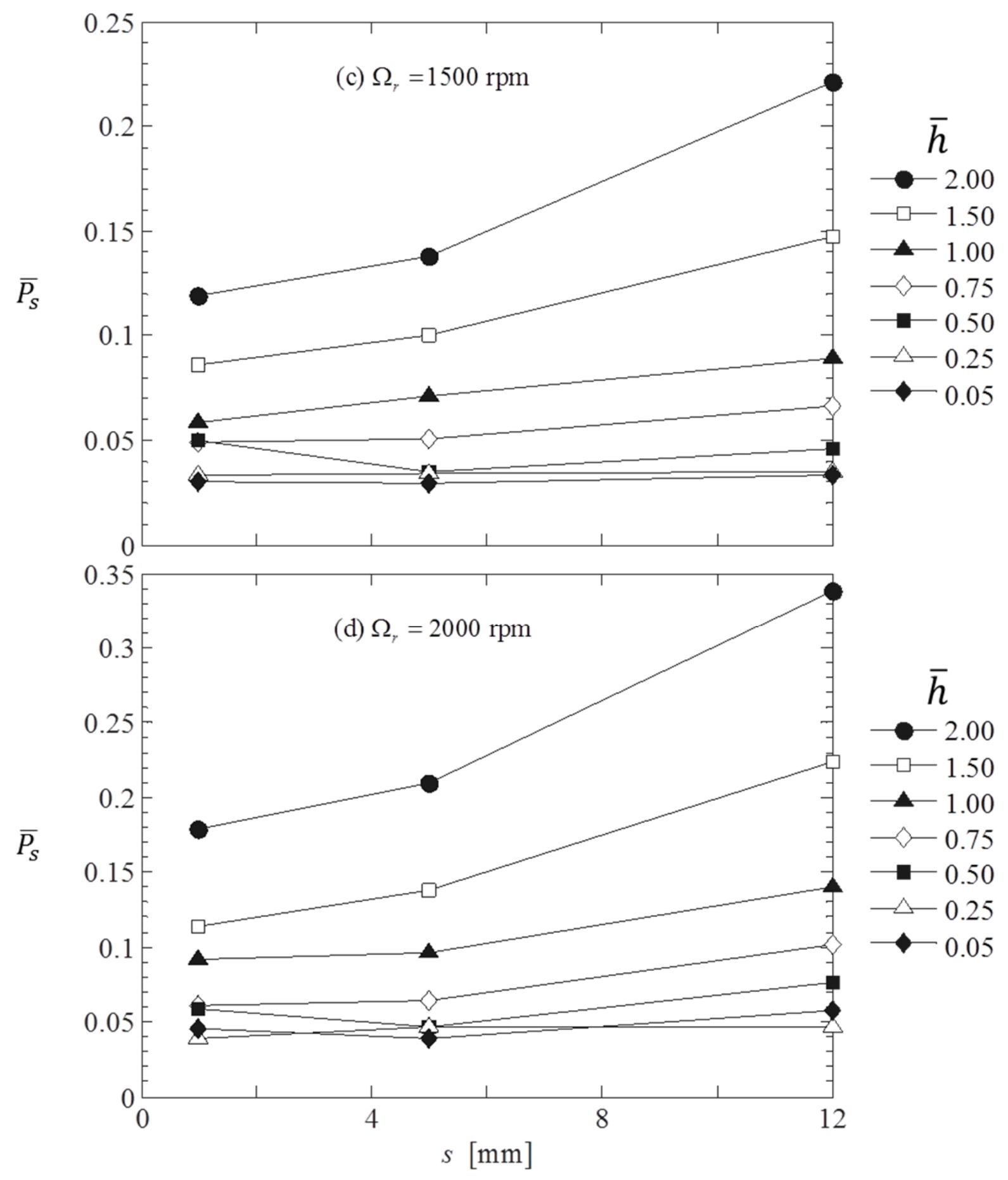



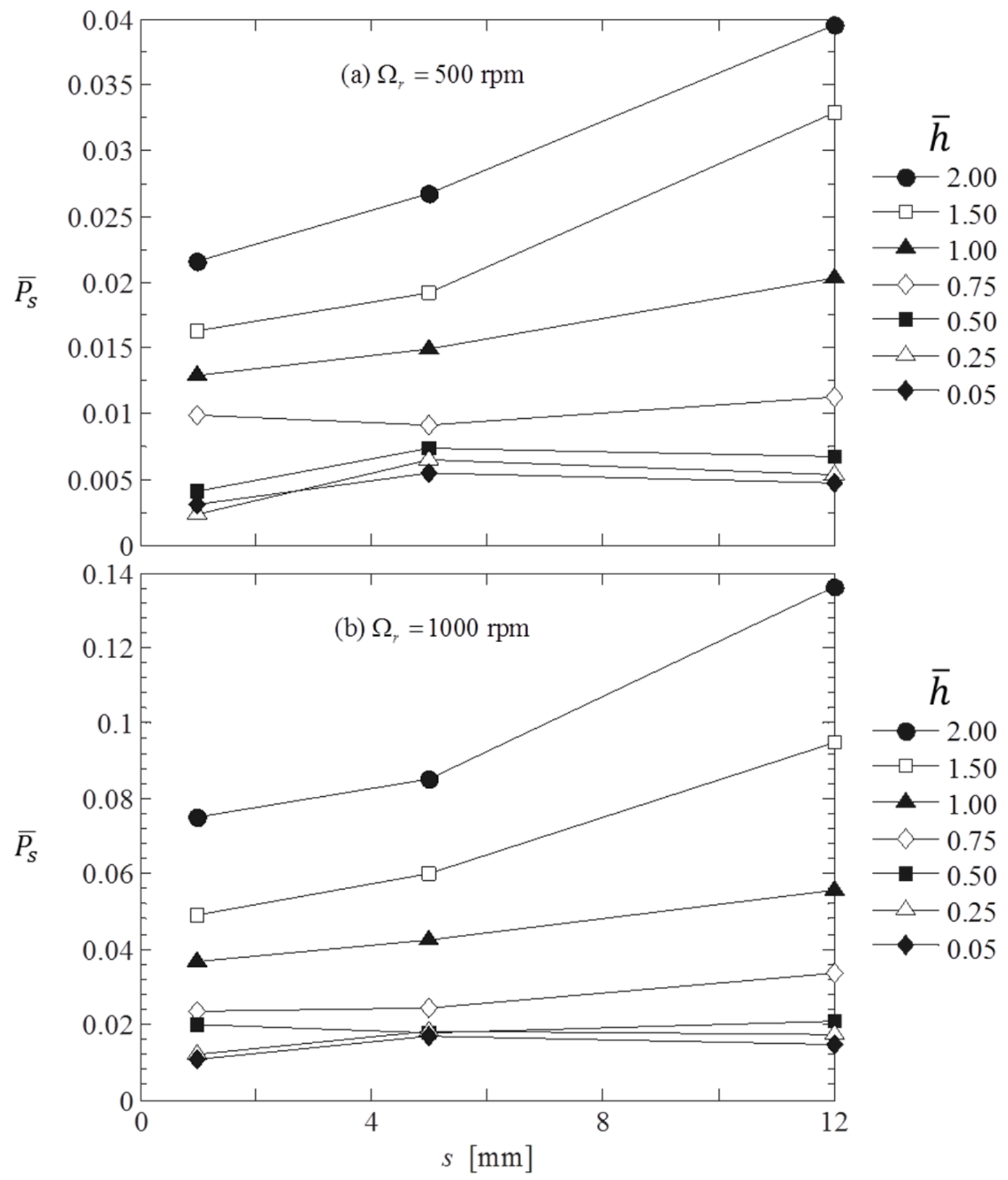

Figure 3.19 Effect of $s$ on $\bar{P}_{S}$ from a ring gear test with $p=5 \mathrm{~mm}$ at (a) $\Omega_{r}=500$ $\mathrm{rpm}$, (b) $\Omega_{r}=1000 \mathrm{rpm},(\mathrm{c}) \Omega_{r}=1500 \mathrm{rpm}$, and (d) $\Omega_{r}=2000 \mathrm{rpm}$. Ring gear \#3 with lubricant $\mathrm{B}$ at $60^{\circ} \mathrm{C}$. (continued) 
Figure 3.19 (continued)

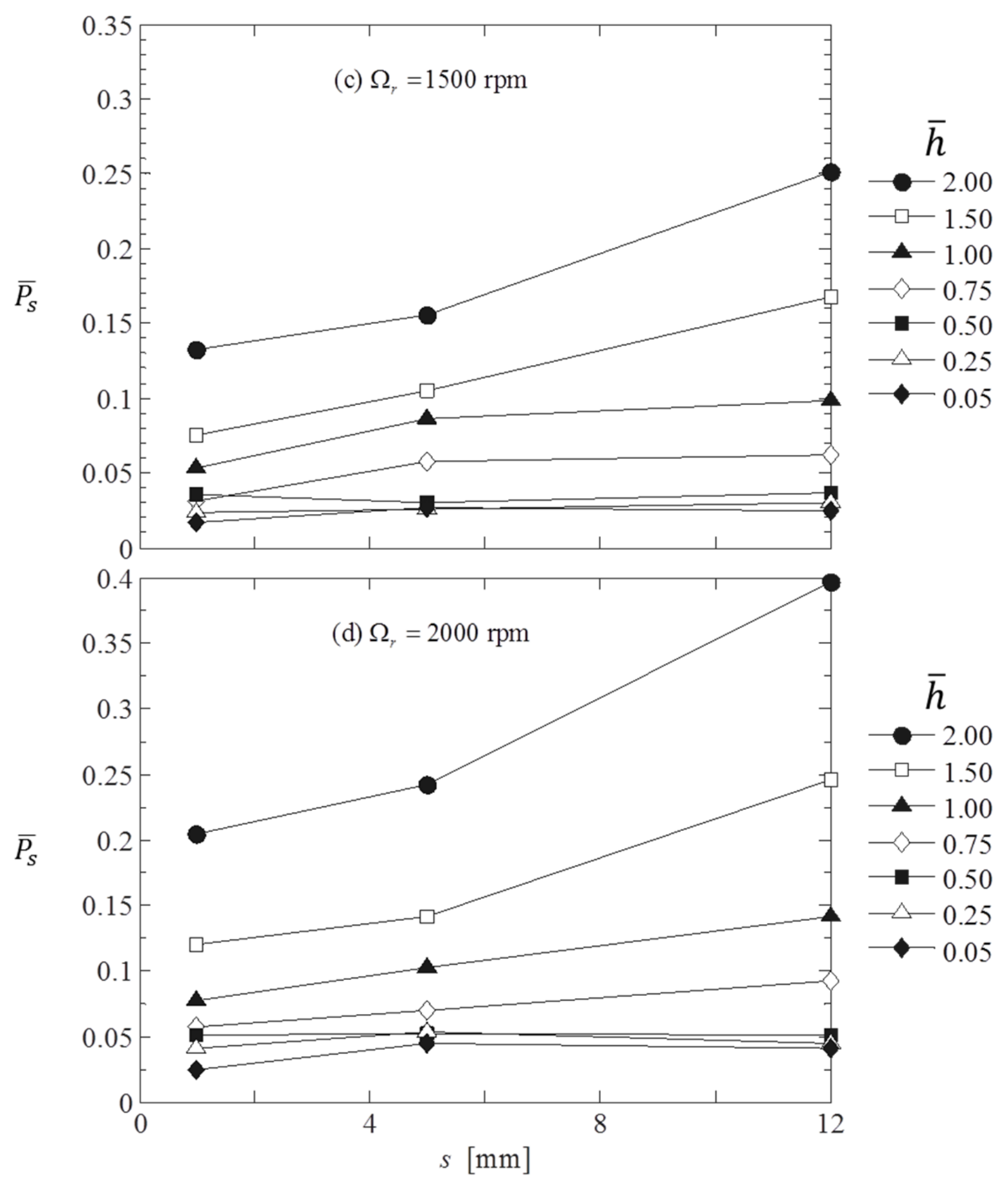




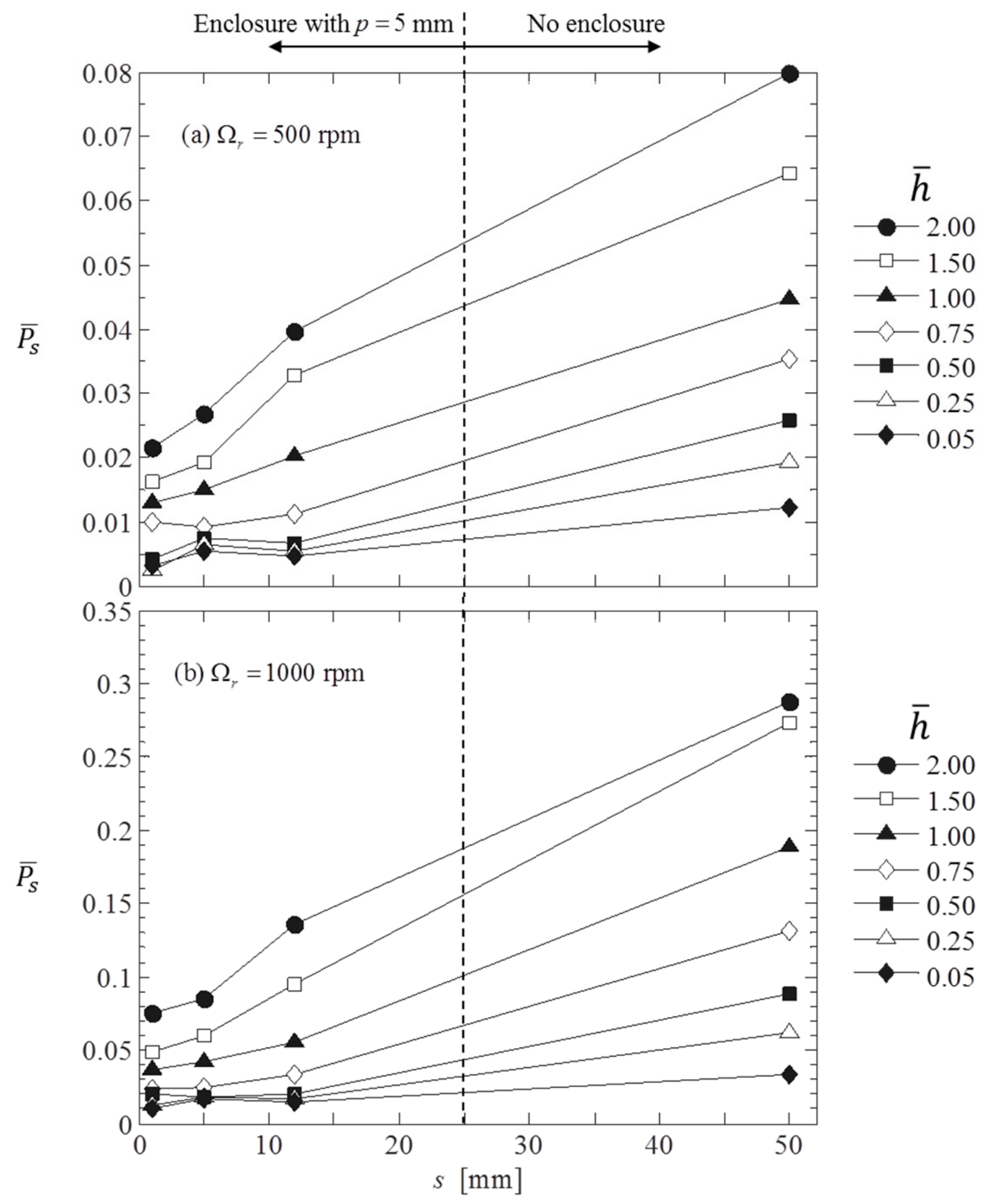

Figure 3.20 Combined effects of $p$ and $s$ on $\bar{P}_{s} \mathrm{~mm}$ at (a) $\Omega_{r}=500 \mathrm{rpm}$ and (b) $\Omega_{r}=1000 \mathrm{rpm}$. Ring gear \#3 with lubricant B at $60^{\circ} \mathrm{C}$. 
Furthermore, for the tightest enclosure used ( $p=5 \mathrm{~mm}$ and $s=1 \mathrm{~mm}$ ) $\bar{P}_{S}$ is $74 \%$ less than the no-enclosure case at $\bar{h}=2.00$ and $\Omega_{r}=1000 \mathrm{rpm}$. As noted above, the volume of oil with enclosures in significantly less than without and further testing is required to make conclusions with confidence.

\subsection{Conclusions}

Chapter 3 presents the data gathered by using the machine/gearbox described in Chapter 2 and following the test matrix presented in Table 2.2. Two main categories of tests were performed, namely single gear tests and gear pair tests. From these testing conditions, the following general trends were observed:

(i) Ring gear diameter has a noticeable effect on spin power loss. Differences in major diameter of the ring gears were small. However, there were consistent differences between the $\bar{P}_{S}$ values for each ring gear. Higher ring gear submersion levels result in higher $\bar{P}_{S}$ due to the larger amount of viscous drag caused by the larger surface area of possible interaction between the oil and gear surfaces.

(ii) Lubricant properties have a significant effect on $\bar{P}_{S}$. Especially, lubricant density was shown to have a large influence on $\bar{P}_{S}$. A higher density lubricant will increase $\bar{P}_{S}$ under all operating conditions. Additionally, lubricant kinematic viscosity was shown to be moderately significant when higher ring gear 
submersion levels were used, where higher kinematic viscosity corresponds to higher $\bar{P}_{S}$.

(iii) Gear teeth are a large contributor to $\bar{P}_{S}$. The presence of gear teeth showed to be hugely significant in not only $\bar{P}_{S}$, but also in the fluid flow behavior within the gearbox. Modeling efforts using the assumption that a rotating gear can be accurately represented by a rotating disk will be inaccurate.

(iv) Pocketing is a sizable portion of $\bar{P}_{S}$. When the effective density of the oil/air mixture pulled into the gear mesh is sufficiently high, pocketing was the dominate component of $\bar{P}_{S}$, especially at higher speeds.

(v) Encasing a gear member provides a great reduction in $\bar{P}_{S}$. The use of enclosures were shown to drastically reduce $\bar{P}_{S}$. The mechanism underlying this effect is not distinguishable with the provided data, however the benefits were significant. 


\section{CHAPTER 4}

\section{SUMMARY AND CONCLUSIONS}

\subsection{Thesis Summary}

An extensive experimental study was conducted to investigate the components of spin power loss of an automotive final drive helical gear pair. A test set-up was developed for this purpose with the ability to spin an unloaded gear pair or a single gear in various operating conditions while measuring torque provided to the gearbox. The test set-up was shown to be capable of producing repeatable power loss measurements within an input ring gear speed range of $\Omega_{r} \in[0,2000] \mathrm{rpm}$ and at bulk lubricant temperatures from $40^{\circ} \mathrm{C}$ to $90^{\circ} \mathrm{C}$.

A test matrix was defined and executed that allowed determination of the main components of spin power loss of the gear box, namely (i) drag loss of the ring gear, (ii) drag loss of the gear shaft bearings/seals, (iii) drag loss of the pinion shaft bearings/seals, and (iv) pocketing losses at the ring-pinion mesh. The test matrix included different lubricants, gear sizes, tooth shapes (including disks with no teeth), single gear and gear 
pair configurations. Input shaft tests with no gears were employed to isolate bearing and seal losses while single ring gear tests were used to quantify ring gear drag losses at various static oil levels. Comparing these sub-system tests to gear pair spin loss tests resulted in the contributions of gear mesh pocketing to overall spin loss.

These tests, that quantified the impact of rotational speed, bulk lubricant temperature, pinion position angle and static lubricant level on spin losses, were extended through design and implementation of various enclosures and shrouds around the ring gear to study the influence of the shape of the surroundings on the gear drag losses.

\subsection{Main Conclusions}

Based on the results presented in Chapter 3, the following technical conclusions can be made in regards to spin power losses in parallel axis gear sets operating within an oil bath:

- Bulk lubricant temperature (i.e. kinematic viscosity) has a sizeable influence on ring gear drag, especially for immersion levels of $\bar{h}>1.0$ and pitch line velocities

( $u_{p}=\frac{2 \pi}{60} r_{p r} \Omega_{r}$ where $r_{p r}$ is the pitch radius of the ring gear) of $u_{p} \geq 12 \mathrm{~m} / \mathrm{s}$ (corresponding roughly to $\Omega_{r} \geq 1000 \mathrm{rpm}$ for the ring gears used in this study). The tests at lower temperatures resulted in consistently higher spin power losses than those at higher temperatures as a direct result of the change in viscosity with temperature. 
- Lubricants having nearly the same viscosity at a given oil temperature were seen to cause different magnitudes of spin losses, primarily due to differences in their density. Lubricant A with the highest density resulted in consistently $15-25 \%$ more power loss compared to lower density fluids B and C.

- The size of a gear, especially its outside diameter, was shown to influence gear drag loss moderately. The size difference between the largest and smallest ring gears tested was only $6 \mathrm{~mm}$ in diameter. With such a size difference, the largest ring gear was measured to cause about $10-15 \%$ more spin loss than the smallest ring gear.

- Spin power losses of a rotating gear with teeth were shown to be significantly larger than a gear blank (or a cylindrical disk) of the same outside diameter. For the ring gear size considered in this study, a spur gear was measured to cause 30 to $70 \%$ more spin power loss than a cylindrical disk with no teeth, indicating clearly that modeling a gear, in a drag loss formulation, as a solid disk will underestimate power losses.

- The test results demonstrate that a helical gear operating at low static lubricant levels causes lower drag spin losses than its spur gear equivalent, while this difference diminishes somewhat for higher $\bar{h}$. The underlying reason between the difference in spur and helical gear behavior is that the flow associated with a helical gear has a significant axial component, resulting in a truly three- 
dimensional flow. This indicates that 2D CFD modeling of helical gear might be flawed.

- Angular position of the pinion around the ring gear, relative to the static oil level, was observed to have a major role in overall spin power loss performance of a gear pair. Fully submerging a gear mesh vastly increases spin power loss of a gear pair especially for $u_{p} \geq 12 \mathrm{~m} / \mathrm{s}$. This is primarily due to an increase in pocketing loss due to higher concentration of the oil-air mixture being squeezed out of the gear mesh interface.

- Ring gear drag was observed to be the most dominant component of spin power loss for static lubricant levels of $\bar{h} \geq 1.0$. Pocketing represents a more sizable portion of total spin power loss when the gear meshing zone experiences higher oil-to-air ratios (i.e. it is at or below the static oil level) and the speed is high $\left(u_{p} \geq 12 \mathrm{~m} / \mathrm{s}\right)$. Below these thresholds, however, pocketing loss was measured to have a secondary role.

- The gearbox spin losses were shown to be influenced by the shape of the gearbox housing, especially in terms of axial and radial distances of housing walls from the rotating gear. Tests with various enclosures that reduce gaps between the ring gear and housing were shown to have consistently lower losses than the test with gearbox only (no filler enclosures), indicating that reducing the empty space around a rotating gear is the most effective method for reducing spin power loss 
of a geared system. Reducing axial clearance between the gear faces and housing walls was shown to be more effective in lowering spin loss than reducing the peripheral (radial) clearance between the gear tips and housing boundary. Experiments were not conclusive enough to suggest that there should be an optimal axial or peripheral clearance value for minimum spin losses. If there is such an optimum gap, it should be a very small one, especially for lower speed applications since the boundary layer thicknesses will be very small. The methodology used here to implement enclosures was not precise enough to operate them in very close locations to the rotating gear.

- Two qualitatively different flow conditions were observed in the churning experiments. Under lower $\bar{h}$ and $u_{p}$ conditions, a dynamic oil level at an elevation below the static oil level was shown to be maintained while complete circulation of the entire volume of oil in the direction of gear rotation was shown

to form (referred to as a vortex in Chapter 3) for higher $\bar{h}$ and $u_{p}$. The latter was shown to correspond to much larger spin losses than the former, in the process adding significant heat generation to the gearbox.

\subsection{Recommendations for Future Work}

This experimental study presented perhaps the most comprehensive database on gear and gearbox spin power losses. However, it made no effort to characterize the influence of bulk lubricant volume on churning loss components. It also did not focus on 
components of power loss components under primarily windage conditions although the gearbox developed in this study would allow such tests. In addition, there might be other gear parameters such as module, face width, and helix angle potentially influencing spin loss. In view of these, the following specific recommendations are proposed for future work:

- Refinement and Expansion of Enclosure Experiments. The data presented in this study utilized only one type of enclosure geometry. Various baffling and flow interrupters should be examined. Running tests with a fully enclosed type enclosure will help with future modeling efforts. Enclosure designs that allow precise adjustment of enclosure gaps are required to collect data that would reveal the influence of gap widths $s$ and $p$ precisely. Additionally, tests utilizing enclosures that do not significantly reduce the volume of oil in the gearbox, yet provide surfaces in close proximity to the gear members would shed light on the influence of boundary layer interactions on spin loss.

- Gearbox Modifications for Increased Bearing Power Loss Measurement Accuracy. The bearing lubrication environment needs to be separated from the test lubrication environment by sealing off the bearing housings and providing dedicated jet lubrication. This would increase the accuracy and repeatability of bearing loss experiments while removing the influence of $\bar{h}$ on bearing spin losses. 
- Investigation of the Effect of Other Relevant Gear Parameters. The test matrix included a helical gear with a helix angle of $33.5^{\circ}$ and a spur gear with zero helix angle. Tests with helical gears having other helix angles are required for quantifying the influence of helix angle on spin losses. Likewise gears with the same outside diameter, but different tooth sizes (module) and face widths should be tested to isolate their influences on spin losses.

- Alternate Lubrication Methods. The gearbox was only operated using dip lubricated conditions. A simple modification to the gearbox would enable a jet lubricated condition. An additional modification would allow for metered flooding of the meshing zone while not having a large impact on ring gear drag. This would also enable much better pocketing characterization with the possible inclusion of oil to air ratio characterization.

- Monitor Oil Pressure within the Gearbox. Studies by Handschuh and Hurrell [15] and Hill [32] suggested that spin power losses are significantly affected by pressure buildup within the gearbox. The addition of pressure transducers in key locations in the gearbox would help shed light on the fluid pressure distributions within the gearbox. 


\section{REFERENCES}

[1] Talbot, D. C., "An Experimental and Theoretical Investigation of the Efficiency of Planetary Gear Sets," Ph.D. Dissertation, The Ohio State University, Columbus, Ohio, 2012.

[2] Kahraman, A., Seetharaman, S., Szweda, T., Kirchner, E., Olsson, M., Bednarek, G., "Development of an Efficiency Model for Transmissions," CTI Conference, Berlin, Germany, December, 2008.

[3] Bednarek, G., Kahraman, A., Seetharaman, S., "Development of an Efficiency Model for Manual Transmissions," VDI-Berichte, Getriebe in Fahrzeugen, Freidrichhafen, Germany, June, 2008.

[4] Szweda, T., "An Experimental Study of Power Losses of an Automotive Manual Transmission,” M.S. Thesis, The Ohio State University, Columbus, Ohio, 2008.

[5] Xu, H., Kahraman, A., Anderson, N. E., Maddock, D., "Prediction of Mechanical Efficiency of Parallel-axis Gear Pairs," Journal of Mechanical Design, 129, 5868, 2007. 
[6] Petry-Johnson, T., Kahraman, A., Anderson, N. E., Chase, D., “An Experimental Investigation of Power Losses of High-speed Spur Gears," Journal of Mechanical Design, 130(6), 062601, 2008.

[7] Li, S., Kahraman, A., "Prediction of Spur Gear Mechanical Power Losses Using a Transient Elastohydrodynamic Lubrication Model," Tribology Transactions, 53, 554-563, 2010.

[8] Talbot, D., Kahraman, A., Singh, A., "An Experimental Investigation of the Efficiency of Planetary Gear Sets," Journal of Mechanical Design, 134, 021003, February 2012.

[9] Daily, J. W., Nece, R. E., “Chamber Dimension Effects on Induced Flow and Frictional Resistance of Enclosed Rotating Disks," Journal of Basic Engineering 82(1): 217-230, 1960.

[10] Mann, R. W., Marston, C. H., "Friction Drag on Bladed Disks in Housings as a Function of Reynolds Number, Axial and Radial Clearance, and Blade Aspect Ratio and Solidity," Journal of Basic Engineering: 719-723, 1961

[11] Akin, L.S., Mross, J.J., "Theory for the Effect of Windage on the Lubricant Flow in the Tooth Spaces of Spur Gears," Journal of Engineering for Industry, 1266$1273,1975$.

[12] Dawson, P H., "Windage Loss in Larger High Speed Gears," Proceedings of the Institution of Mechanical Engineers: 198(1): 51-59, 1984. 
[13] Lord, A. A., "An Experimental Investigation of Geometric and Oil Flow Effects on Gear Windage and Meshing Losses," PhD Thesis, University of Wales, Swansea, 1998.

[14] Al-Shibl, K., Simmons, K., Eastwick, C. N., "Modeling Windage Power Loss from an Enclosed Spur Gear," Proceedings of the Institution of Mechanical Engineers, Part A: Journal of Power and Energy 221(3): 331-341, 2007.

[15] Handschuh, R. F., Hurrell, M. J., "Initial Experiments of High-Speed Drive System Windage Losses," International Conference on Gears, Garching, Germany, 2011.

[16] Hill, M. J., Kunz, R. F., Noack, R. W., Long, L. N., Morris, P., Handschuh, R. F., "CFD Technology for Rotorcraft Gearbox Windage Aerodynamics Simulation," Gear Technology, 2008.

[17] Seetharaman, S., Kahraman, A., "A Windage Power Loss Model for Spur Gear Pairs," Tribology Transactions, 53(4), 473-484, 2010.

[18] Seetharaman, S, Kahraman, A., "Load-Independent Power Losses of a Spur Gear Pair: Model Formulation,” Journal of Tribology, 131, $022201,2009$.

[19] Seetharaman, S., Kahraman, A., Moorhead, M., Petry-Johnson, T., "LoadIndependent Power Losses of a Spur Gear Pair: Experiments and Model Validation," Journal of Tribology, 131, 022202, 2009. 
[20] Terekhov, A.S., "Basic Problems of Heat Calculation of Gear Reducers," Proceedings of the JSME International Conference on Motion and Power Transmissions, Hiroshima, Japan, Nov. 23-26, pp. 490-495, 1991.

[21] Boness, R.J. “Churning Losses of Discs and Gears Running Partially Submerged in Oil," Proceedings of the ASME International Power Transmission and Gearing Conference, Chicago, Illinois, pp. 355-359, 1989.

[22] Luke, P., Olver, V., "A Study of Churning Losses in Dip-Lubricated Spur Gears," Proceedings of the Institution of Mechanical Engineers, Part G: Journal of Aerospace Engineering 213(5): 337-346, 1999.

[23] Petry-Johnson, T. T., "Experimental Investigation of Spur Gear Efficiency,” M.S. Thesis, The Ohio State University, Columbus, Ohio, 2007.

[24] Moorhead, M., "Experimental Investigation of Spur Gear Efficiency and the Development of a Helical Gear Efficiency Test Machine," M.S. Thesis, The Ohio State University, Columbus, Ohio, 2008.

[25] Changenet, C., Velex, P., "A Model for the Prediction of Churning Losses in Geared Transmissions_-Preliminary Results," Journal of Mechanical Design 129(1): 128-133, 2007.

[26] Changenet, C., Velex, P., "Housing Influence on Churning Losses in Geared Transmissions," Journal of Mechanical Design 130(6): 062603, 2008. 
[27] Pechersky, M. .J., Wittbrodt, M. J., “An Analysis of Fluid Flow Between Meshing Spur Gear Teeth," Proceedings of the ASME Fifth International Power Transmission and Gearing Conference, Chicago, Illinois, pp. 335-342, 1989.

[28] Diab, Y., Ville, F., Houjoh, H., Sainsot, P., Velex, P., "Experimental and Numerical Investigations on the Air-Pumping Phenomenon in High-Speed Spur and Helical Gears," Journal of Mechanical Engineering Science 219(8): 785-800, 2005.

[29] Talbot, D., Kahraman, A., Seetharaman, S., “A Pocketing Power Loss Model for Helical Gears," in review, Journal of Tribology, 2013.

[30] Chase, D., "Development of an Efficiency Test Methodology for High-Speed Gearboxes," M.S. Thesis, The Ohio State University, Columbus, Ohio, 2005.

[31] Sistani, E., "PIV Measurements Around a Rotating Single Gear Partially Submerged in Oil Within Modeled SAAB Gearbox," M.S. Thesis, Chalmers University of Technology, 2010.

[32] Hill, M. J., “A Computational Investigation of Gear Windage.” PhD Dissertation, the Pennsylvania State University, State College, Pennsylvania, 2010.

[33] Seetharaman, S., Kahraman, A., "A model to Predict Effect of Enclosures on Spin Losses of a Gear," in preparation for the Journal of Tribology, 2013. 
[34] Hilty, D., “An Experimental Investigation of Spin Power Losses of Planetary Gear Sets,” M.S. Thesis, The Ohio State University, Columbus, Ohio, 2010.

[35] Harris, T. A., Kotzalas, M. N., Rolling Bearing Analysis, 5th ed., CRC Press, New York, 2007

[36] SKF Product Detail from Online Catalog $<$ http://www.skf.com/us/products/productdetail.html?prodid=1050010006>

[37] SKF Product Detail from Online Catalog $<$ http://www.skf.com/us/products/productdetail.html?prodid=1050010007> 